\title{
Objective Observer-Relative Flow Visualization in Curved Spaces for Unsteady 2D Geophysical Flows
}

Peter Rautek, Matej Mlejnek, Johanna Beyer, Jakob Troidl, Hanspeter Pfister, Thomas TheußI, and Markus Hadwiger
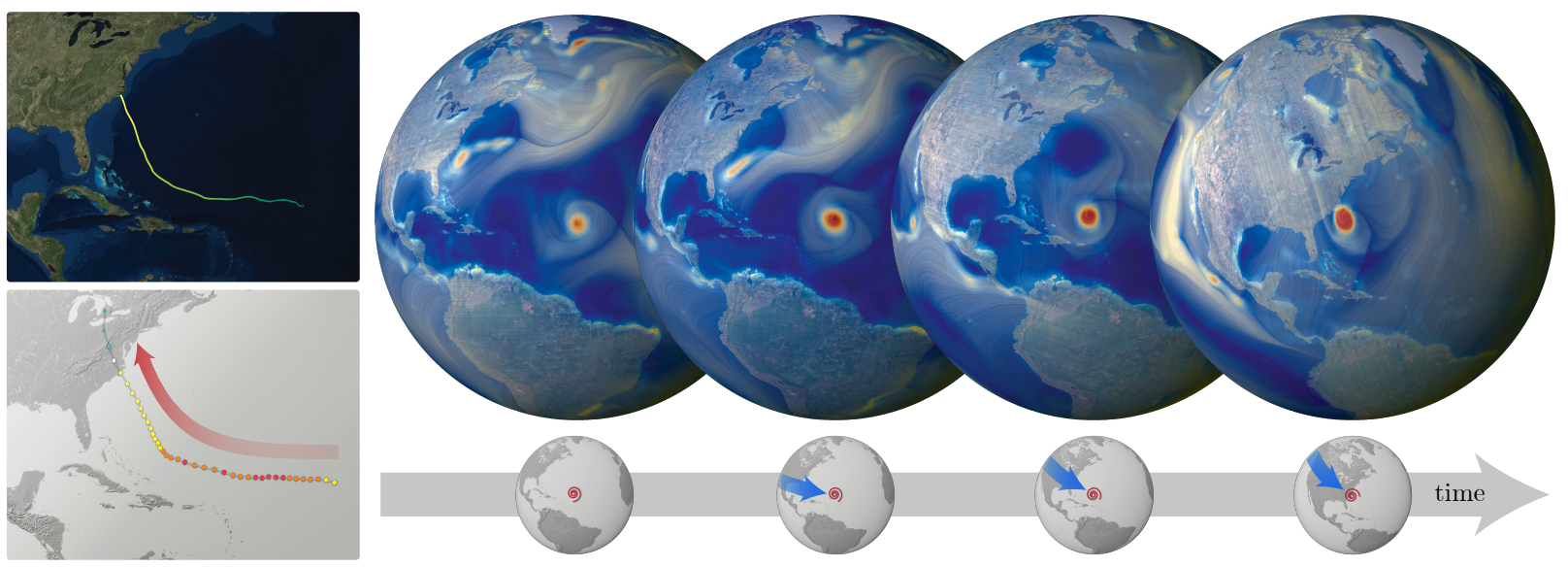

Fig. 1. Observer motion relative to the time evolution of features: Hurricane Isabel in a time-dependent global wind data set. (Bottom left) The actual path of Isabel (from NHC/Wikipedia). (Top left) Our observer field $\mathbf{u}$ automatically follows the motion of Isabel without explicit tracking of its path. The shown path is simply a path line of $\mathbf{u}$. (Right) Feature-relative visualization, focused on Isabel in the center, enabling analysis of its time evolution "in place." The hurricane appears steady, with the Earth moving inversely underneath.

\begin{abstract}
Computing and visualizing features in fluid flow often depends on the observer, or reference frame, relative to which the input velocity field is given. A desired property of feature detectors is therefore that they are objective, meaning independent of the input reference frame. However, the standard definition of objectivity is only given for Euclidean domains and cannot be applied in curved spaces. We build on methods from mathematical physics and Riemannian geometry to generalize objectivity to curved spaces, using the powerful notion of symmetry groups as the basis for definition. From this, we develop a general mathematical framework for the objective computation of observer fields for curved spaces, relative to which other computed measures become objective. An important property of our framework is that it works intrinsically in 2D, instead of in the 3D ambient space. This enables a direct generalization of the 2D computation via optimization of observer fields in flat space to curved domains, without having to perform optimization in 3D. We specifically develop the case of unsteady 2D geophysical flows given on spheres, such as the Earth. Our observer fields in curved spaces then enable objective feature computation as well as the visualization of the time evolution of scalar and vector fields, such that the automatically computed reference frames follow moving structures like vortices in a way that makes them appear to be steady.
\end{abstract}

Index Terms-Flow visualization, observer fields, frames of reference, objectivity, symmetry groups, intrinsic covariant derivatives

\section{INTRODUCTION}

Flow visualization is concerned with velocity vector fields that describe fluid motion. Since the definition of velocity is the infinitesimal change of spatial position with respect to time, this description depends on

- Peter Rautek, Matej Mlejnek, and Markus Hadwiger are with King Abdullah University of Science and Technology (KAUST), Visual Computing Center, Thuwal, 23955-6900, Saudi Arabia.

E-mail: \{peter.rautek, matej.mlejnek, markus.hadwiger\}@kaust.edu.sa.

- Johanna Beyer and Hanspeter Pfister are with Harvard University, Cambridge, MA, USA. E-mail: \{jbeyer, pfister\}@ seas.harvard.edu

- Jakob Troidl is with King Abdullah University of Science and Technology (KAUST), Visual Computing Center, Thuwal, 23955-6900, Saudi Arabia, and TU Wien, Vienna, Austria. E-mail: jakob.troidl@googlemail.com.

- Thomas Theußl is with King Abdullah University of Science and Technology (KAUST), Core Labs, Thuwal, 23955-6900, Saudi Arabia.

E-mail: thomas.theussl@kaust.edu.sa.

Manuscript received xx xxx. 201x; accepted xx xxx. 201x. Date of Publication xx xxx. 201x; date of current version xx xxx. 201x. For information on obtaining reprints of this article, please send e-mail to: reprints@ieee.org. Digital Object Identifier: $x x . x x x x / T V C G .201 x . x x x x x x x$ the notion of what is considered to be "the same position" over time. Mathematically, this is the notion of a frame of reference, or an observer. A frame of reference is a way of referencing positions in space over time, i.e., a time-dependent transformation of space, with respect to which derivatives are to be taken. (Not an observer as an actual person.)

For this reason, flow visualization as well as the computation of flow features, such as vortices, in general inherently depend on the chosen frame of reference. An important related concept therefore is invariance with respect to certain changes of reference frame. Galilean invariance [28] refers to frame changes with constant relative velocity, which is inherently related to inertial frames in physics [63]. Objectivity [31] considers frame changes with time-dependent translations and rotations. The standard definition used in visualization and continuum mechanics is the one of Truesdell and Noll [65], which has received significant attention for flow fields since the work of Haller [31]. A major basic limitation of this definition, however, is that it is given only for Euclidean space and cannot be used in general curved spaces.

One area where objectivity is particularly relevant is that of geophysical flows, such as in the Earth's atmosphere or oceans. The natural reference frame, in this case, is rotating [51, p.14], i.e., non-inertial, and Galilean invariance is not well-suited. As is common in flow visualization, we only consider motions by themselves (kinematics), not 
the forces generating them (dynamics). We can therefore consider accelerating reference frames just as easily as inertial frames, because the differences in the forces acting are irrelevant. For example, in Fig. 2 we consider an airplane as an observer measuring motion relative to its own reference frame. Naturally, this does not change a phenomenon such as a hurricane, but it does change all relative velocity measurements.

Although the Earth's surface is embedded in a flat 3D space (ignoring relativistic effects), it is beneficial to be able to view the domain where a geophysical flow field is defined from an intrinsic, curved 2D perspective. This not only allows for simplified computations in 2D instead of in 3D, or considering different altitude or depth layers separately; it also corresponds perfectly to the reference frame of circular orbits, such as those of satellites or airplanes moving along great circles around the Earth, as shown in Fig. 2. For visualization purposes, it is also common to visualize spherical layers. Moreover, scientists have even discovered that some geophysical fluid systems, such as aspects of our atmosphere or even that of Jupiter $[29,56]$, can sometimes be modeled in a more realistic way in $2 \mathrm{D}$ than in $3 \mathrm{D}$, because the atmosphere is so much thinner than wide. For this reason, it can be crucial to perform computations in $2 \mathrm{D}$, because the physical behavior of $2 \mathrm{D}$ turbulence can be radically different from 3D turbulence [23,35, 40].

With this motivation, we develop general techniques for flow visualization and computation of flow features in curved spaces. For practical relevance, we illustrate results for geophysical flows on spheres. We generalize the notion of observer fields [30] from Euclidean space to curved spaces. This enables "following" flow features such as vortices, and visualizing the corresponding time evolution of scalar and vector attributes "in place." For example, Fig. 1 shows hurricane Isabel (2003) in a time-dependent global wind simulation. Our computed observer field automatically follows the motion of the hurricane. Fig. 1 (right) shows feature-relative visualization, where the observer field makes the motion of Isabel appear to be steady. This enables "feature-centric" analysis, without having to explicitly follow the motion of the hurricane.

\subsection{Observer Fields and Observed Time Derivatives}

An observer field $\mathbf{u}(x, t)$ is a time-dependent velocity field that, instead of describing the motion of particles, describes the motion of a continuous field of observers [30]. The path lines of the observer field correspond to the world lines [3, p.8] of individual spatial locations in the space of an observer that are viewed as being "the same point" over time. If an observer field $\mathbf{u}$ describes a rigid motion, it is a Killing vector field [53]. Figs. 2 (a,b,c) depict three time steps of observer fields on a sphere. Fig. 3 shows one example input vector field $\mathbf{v}$ describing a simple rotating vortex, as it is seen by four different observer fields $\mathbf{u}$.

The observed time derivative corresponding to a given $\mathbf{u}(x, t)$ measures the differential change of an arbitrary input field $\mathbf{v}(x, t)$ with respect to the observer motion described by $\mathbf{u}$. This concept was introduced in the context of Euclidean space [30]. We define this time derivative, on any differential manifold $M$, as the differential operator

$$
\frac{\mathscr{D}}{\mathscr{D} t}:=\frac{\partial}{\partial t}+\mathscr{L}_{\mathbf{u}}
$$

Here, $\mathscr{L}_{\mathbf{u}}$ denotes the Lie derivative [22, Ch. 4] with respect to the observer field $\mathbf{u}$. In order to "steadify" an input vector field v, e.g., to be able to compute and follow features, we compute observer fields $\mathbf{u}$ by minimizing the derivative $(\mathscr{D} / \mathscr{D} t)(\mathbf{v}-\mathbf{u})$. If Fig. 3 is interpreted as showing four different input fields $\mathbf{v}$, the observer-relative visualization with respect to the optimized field $\mathbf{u}$ will always look like Fig. 3 (d).

\subsection{Mathematical Framework for Curved Spaces}

We develop a novel fully intrinsic mathematical framework for 2D flow visualization and feature computation in curved spaces. Our major contribution is the generalization of a prior Euclidean framework [30] for the objective computation of observer fields to curved spaces. We compute these fields by solving an energy minimization problem, mak ing unsteady input flows as steady as possible. We first introduce a generalized notion of objectivity for curved spaces. While equivalent to the standard one in Euclidean space [65], ours is fully intrinsic, using the concepts of continuous symmetry groups and group actions on

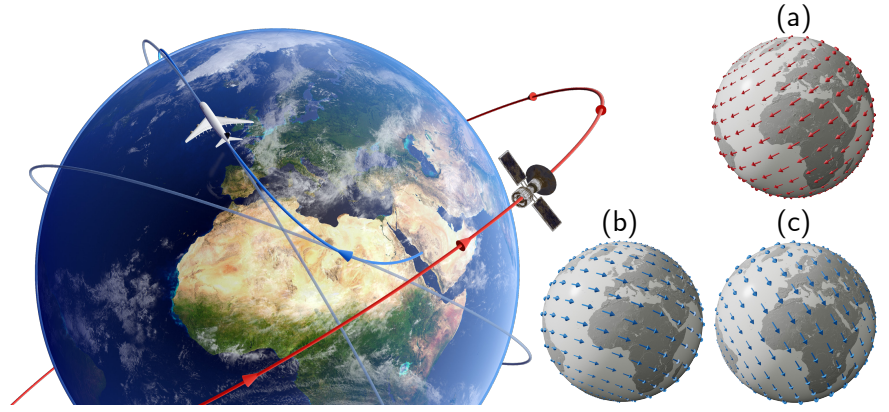

Fig. 2. Observers in curved spaces. An airplane (blue path) and a satellite (red path) are moving relative to the Earth. At each instant in time, with respect to the Earth's surface both fly in the direction of a geodesic (a great circle). Considering the observer (airplane or satellite) as stationary, the instantaneous relative motion of the Earth is a Killing vector field on the sphere, in the opposite direction (right). (a) The Killing field for the satellite is a steady field (assuming the satellite ground track is always the same geodesic); (b,c) The field for the airplane is unsteady: Each instant in time is determined by a different tangential velocity.

manifolds from mathematical physics. From general symmetry groups, we specialize to observer transformations modeled as elements of the isometry group of a manifold $M$. To simplify computations, we work with infinitesimal isometries, given explicitly by vector fields on $M$.

To be able to achieve this, we derive the differential geometric operators needed to compute observer fields in curved spaces: (1) Observed time derivatives; (2) Approximate Killing fields; and (3) The velocity gradient tensor $\nabla \mathbf{v}$ as a covariant derivative [22, Ch. 9], instead of the Jacobian that is commonly used in flow visualization.

Methodology. Our generalization of objectivity and observer fields to curved spaces requires mathematical machinery from Riemannian geometry and mathematical physics that is not common in the flow visualization literature. For this reason, we summarize background and our notation in the beginning, and provide extensive supplementary appendixes. These techniques enable deriving a powerful generalization of previous flow visualization techniques from flat space to curved spaces in a clean and intrinsic manner. We believe that this powerful methodology can be very useful for flow visualization in general, and that it is worthwhile to introduce it to the flow visualization literature.

\section{Related Work}

The framework presented here extends the work of Hadwiger et al. [30] from Euclidean space $\mathbb{R}^{n}$ to curved spaces, focusing on curved twomanifolds. We also model a collection of observers by an observer velocity field describing their motion, which we also compute by solving a least-squares problem such that (1) it is objective, and (2) the input flow field is as steady as possible relative to the observer field. However, the generalization to curved spaces is conceptually and technically challenging. We propose a fully intrinsic generalization, working "inside" the curved space. This results in natural and efficient generalizations, and facilitates computing 2D observer fields via $2 \mathrm{D}$ optimization.

Flow visualization on curved surfaces. All major flow visualization techniques have been extended from flat space to curved surfaces. For example, LIC [14] and variants $[43,55,60]$ have been extended to curved surfaces, either for parametric surfaces [21], by texturing triangle meshes $[8,59,62]$, or by computing LIC in screen space $[6,36]$. Image-based texture advection techniques $[37,67]$ have been extended as well, such as image-based flow visualization on surfaces $[39,68]$. Image space techniques typically project vector fields from $3 \mathrm{D}$ to $2 \mathrm{D}$ screen space for visualization. We refer to the survey by Edmunds et al. [20]. Texture atlases have also been used for flow visualization, for example flow charts [44]. We similarly use multiple coordinate charts.

Vector field design on surfaces. We extensively use differential properties of manifolds and vector fields. These are particularly important for computations with vector fields on surfaces, such as vector field design on surfaces $[15,16,71]$. We refer to the survey of Peng and Laramee [52]. We would like to particularly point out the use of geodesic polar maps and parallel transport along geodesics in the work 

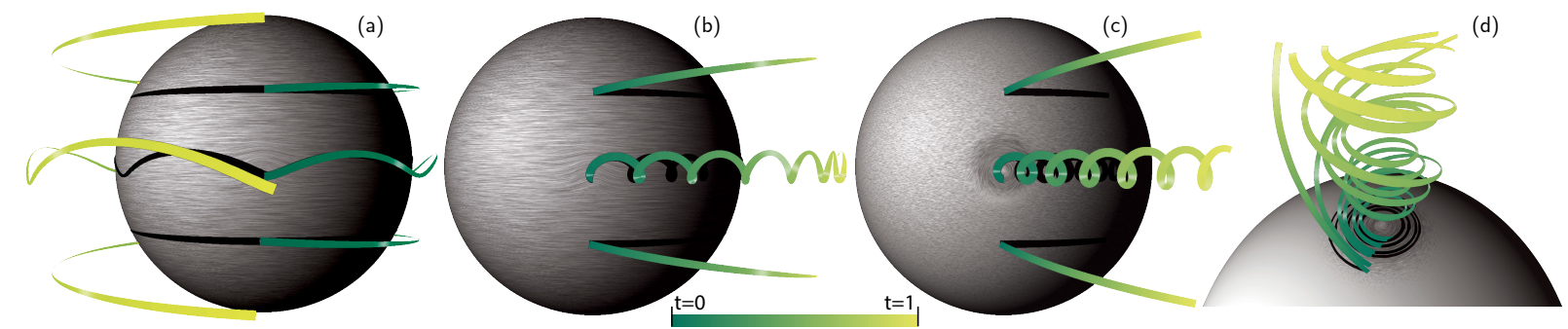

Fig. 3. A rotating vortex seen by four different observers. The observers rotate leftward around the sphere (here, around a vertical axis), with angular velocities $\boldsymbol{\omega}=-2 \pi,-\pi / 4,-\pi / 8,0\left[\mathrm{~s}^{-1}\right]$ (a,b,c,d). All path lines are on the sphere (the depicted shadows), but we visualize the progression of time as increasing radial distance and color (see inset). If these are seen as four different input fields $\mathbf{v}$, minimizing the observed time derivative $\mathscr{D} / \mathscr{D} t(\mathbf{v}-\mathbf{u})$ gives observer fields $\mathbf{u}$ with rightward rotations $\boldsymbol{\omega}=2 \pi, \pi / 4, \pi / 8,0\left[s^{-1}\right]$. (d) Observer-relative visualization is the same for all four cases.

of Palacios and Zhang [50]. The notion of covariant derivative, which we employ in our work, is inherently related to parallel transport.

Covariant derivatives have been used for mesh processing [18] and computing vector [17] and direction [69] fields on surfaces. An important focus is often their discretization on triangle meshes [5], e.g., by using discrete exterior calculus [18]. We discretize on triangle vertices in 2D charts, and particularly focus on fully intrinsic 2D computations.

Interpolation. We use standard barycentric interpolation in triangles. However, spherical barycentric coordinates, i.e., mean value coordinates on the sphere, could also be used for better interpolation [38].

Killing vector fields on (Riemannian) manifolds [19] correspond to their infinitesimal isometries. Their flows correspond to the intrinsic isometries of a manifold. Basics of Killing fields for curved surfaces are discussed by Ben-Chen et al. [9] and by Solomon [57]; a more advanced treatment is given by Petersen [53]. Approximate Killing fields have been used to compute approximate intrinsic isometries of curved surfaces [9], or to design approximate Killing fields on meshes [4,5]. As-rigid-as-possible shape interpolation methods [1] work directly with isometries instead of with derivatives, and are computed extrinsically.

Observers. The concept of an observer as well as that of objectivity in Euclidean space has been recognized to be of importance in fluid mechanics [31] as well as in flow visualization [28]. A standard reference in continuum mechanics is Truesdell and Noll [65], whose definition for objectivity in Euclidean space is used often [7, 25, 31, 32, 34,48].

Vortex detection and frame invariance. Vortices are an important topic in fluid mechanics [54] and in flow visualization [28]. Many vortex detection methods were originally defined for steady flow, such as Sujudi and Haimes [61], and were later extended to unsteady flow [70]. Galilean invariance of these critera is considered to be important [28]. Several known methods are Galilean-invariant, such as Weinkauf et al. [70], or the method of Bujack et al. [13]. The latter jointly considers multiple observers for 2D flow fields. Specific vortex detectors have been developed to be objective by design [31,32]. Günther et al. [7, 25] present objective methods for Euclidean space that are generic by building on optimization, making non-objective vortex detectors objective. Other kinds of invariance have been defined, particularly for flow fields, such as rotation invariance [26], or hyper-objective vortices [27].

Flow decomposition methods, such as variants of the HelmholtzHodge decomposition [10,11], can also be used to remove background flow, corresponding to the harmonic component [12]. These decompositions can be computed on curved surfaces [64]. However, they apply only to time-independent fields, or alternatively they have to be applied to each time step individually, without considering time derivatives.

\section{BACKGROUND AND NOTATION}

Our intrinsic framework enables all computations, most importantly the optimization of observer fields, to be 2D for curved surfaces, instead of requiring 3D ambient space computations. We perform all computations in an atlas of 2D coordinate charts (Fig. 4). This also simplifies visualization computations on curved surfaces, by working directly in charts, for example for path line integration or Line Integral Convolution (LIC), as depicted in Fig. 3. However, already a surface as simple as a sphere cannot be covered by a single chart. All computations in charts therefore need to be independent of the choice of coordinates. We ensure this invariance by using general tensor methods [24], with the corresponding transformation rules for transitions between charts.
Vector fields. A smooth vector field $\mathbf{v}$ on a manifold $M$ is a smooth function giving a vector $\mathbf{v}(x)$ at every point $x \in M$ on the manifold, i.e.,

$$
\begin{aligned}
\mathbf{v}: M & \rightarrow T M, \\
x & \mapsto \mathbf{v}(x) .
\end{aligned}
$$

$T M$ refers to the tangent bundle of $M$, the manifold of all tangent spaces of $M$, and a vector field is also referred to as a section of $T M$. Most important, $\mathbf{v}(x)$ is an element of the tangent space at $x$, denoted by $T_{x} M$. Where no confusion arises, we also denote a single vector $\mathbf{v}(x)$ by $\mathbf{v}$.

Coordinate charts map open subsets $U \subset M$ of an $n$-dimensional manifold $M$ to $\mathbb{R}^{n}$. Coordinates on a curved manifold $M$ are thus not given by coordinate vectors, which is only possible in linear spaces, but for each chart by $n$ coordinate functions $\left\{x^{i}\right\}$, with each $x^{i}: U \rightarrow \mathbb{R}$. For example, for $n=2$, the coordinate functions $x^{1}, x^{2}$, or, sometimes, $u, v$. In contrast, basis vectors live in each tangent space $T_{x} M$, at $x \in M$.

Bases. Vectors are geometric objects independent of any chosen basis. In order to refer a vector $\mathbf{v}$ to a basis, we expand it in components as $\mathbf{v}=v^{i} \mathbf{e}_{i}$, where $\left\{\mathbf{e}_{i}\right\}$ is a basis for $T_{x} M$, and, in general, the $T_{x} M$ at different $x \in M$ have different bases. We employ the Einstein summation convention [22, p.59], implying summation over indices occurring twice (once "upstairs" and "downstairs" each), e.g., $v^{i} \mathbf{e}_{i}:=\sum_{i} v^{i} \mathbf{e}_{i}$.

Dual bases. We will also need the concept of a dual basis $\left\{\omega^{i}\right\}$, where $\omega^{i}\left(\mathbf{e}_{j}\right)=\delta_{j}^{i}$, with the Kronecker delta $\delta_{j}^{i}=1$ if $i=j$, and zero otherwise. Each $\omega^{i}$ is a covector, or 1-form, which is a linear function mapping a vector to a scalar. The dual basis $\left\{\omega^{i}\right\}$ reads off the components of a vector $\mathbf{v}$ referred to $\left\{\mathbf{e}_{i}\right\}$, such that $\mathbf{v}=\omega^{i}(\mathbf{v}) \mathbf{e}_{i}$.

Metrics. Lengths and angles of vectors are determined in an intrinsic manner by defining a (Riemannian) metric $\mathbf{g}$ on $M[19, \mathrm{p} .35]$, which is a second-order tensor field defining an inner product $\langle\mathbf{v}, \mathbf{w}\rangle:=\mathbf{g}(\mathbf{v}, \mathbf{w})$ on each $T_{x} M$, varying smoothly over $M$. The vector norm is then defined as $\|\mathbf{v}\|:=\langle\mathbf{v}, \mathbf{v}\rangle^{1 / 2}$. Computations with $\mathbf{g}$ can use components $g_{i j}:=\left\langle\mathbf{e}_{i}, \mathbf{e}_{j}\right\rangle$ referred to $\left\{\mathbf{e}_{i}\right\}$, computing $\langle\mathbf{v}, \mathbf{w}\rangle$ as $g_{i j} v^{i} w^{j}$. We also extensively use covariant derivatives compatible with a given metric $\mathbf{g}$

Velocity gradients. An important derived quantity of a velocity vector field $\mathbf{v}$ is the velocity gradient tensor field $\nabla \mathbf{v}$. In flow visualization, $\nabla \mathbf{v}$ is often seen as a Jacobian $\partial_{j} v^{i}$ of partial derivatives in Cartesian coordinates. However, these coordinates do not exist in curved spaces, and in general $\nabla \mathbf{v} \neq\left(\partial_{j} v^{i}\right) \mathbf{e}_{i} \otimes \omega^{j}$, since $\partial_{j} v^{i}$ is not tensorial [22, p.241].

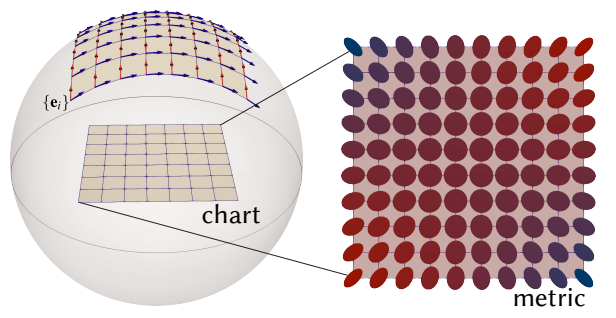

Fig. 4. Our intrinsic framework employs a perspective from within a curved manifold $M$. Thus, all computations on curved surfaces are 2D computations in 2D coordinate charts. Each point $x \in M$ has intrinsic differential properties: A (Riemannian) metric, and covariant derivative operators. A vector field $\mathbf{v}$ is a vector $\mathbf{v}(x)$ in each tangent space $T_{x} M$. Per chart, each $T_{x} M$ has a coordinate basis $\left\{\mathbf{e}_{i}\right\}$, and a dual basis $\left\{\omega^{i}\right\}$. The symmetric metric tensor field is visualized with glyphs on the right. 
We note that even in flat $\mathbb{R}^{2}$ with polar coordinates, $\partial_{j} v^{i}$ is not a tensor. This has important implications, such as that a tensor that vanishes in one coordinate system must vanish in all coordinate systems [24, p.82]. This is not true for $\partial_{j} v^{i}$, and thus the Jacobian behaves only as it should in special cases, such as by restriction to Cartesian tensors [2, Ch.2].

Our framework requires a more general definition of $\nabla \mathbf{v}$ as the covariant derivative $\nabla_{j} v^{i}$, which is standard in mathematical physics. However, we have found no explicit use of this concept in the flow visualization literature. We derive this notion for our purposes in Sec. 6 .

Approximate observer Killing fields. In general, an observer field is allowed to be given by an arbitrary vector field $\mathbf{u}$. However, it makes sense to restrict the motion described by $\mathbf{u}$ to be "as rigid as possible." We therefore optimize observer fields such that they correspond to approximately isometric deformations, i.e., to "almost-rigid" motions. In the same vein as Hadwiger et al. [30], we minimize the rate-ofdeformation tensor of $\mathbf{u}$. However, in curved spaces $M$ this computation requires using the covariant derivative $\nabla \mathbf{u}$, and the metric $\mathbf{g}$ on $M$.

\section{Observers in Curved Spaces}

The first crucial notion for us to define is the meaning of an observer in a curved space. In analogy with the standard notion of observer transformations in Euclidean space [65], it is natural to consider observer transformations to in general be time-dependent distance-preserving transformations of the underlying space, i.e., time-dependent isometries. In the general case, however, we have to consider the intrinsic isometries of a smooth manifold $M$ with metric $\mathbf{g},{ }^{1}$ corresponding to geodesic distances instead of Euclidean distances. Generally stated,

Remark. We employ a (non-relativistic) concept of frames of reference postulating that all observers agree on the pairwise spatial geodesic distances of events happening at the same time, and all observers also agree on the time difference between events (i.e., time is absolute).

Observer transformations are therefore time-dependent intrinsic distance-preserving transformations. Mathematically, we model these observer transformations as paths $t \mapsto g(t) \in G$, through a Lie group $G$, chosen as the isometry group $G:=\operatorname{Isom}(M)$ of the manifold $M$ with metric g. In our framework, we often also work directly with the derivatives of $g(t)$, forming the corresponding path $t \mapsto X(t) \in \mathfrak{g}$ through the Lie algebra $\mathfrak{g}=\mathfrak{i s o m}(M)$, the Lie algebra of infinitesimal isometries.

\subsection{Observers on the Sphere}

Figs. 2 and 5 illustrate this idea for $M=\mathbb{S}^{2}$, the standard two-sphere. The motion of the Earth relative to a fixed observer, such as the airplane in Fig. 2, is given by a path of isometries $t \mapsto g(t) \in \mathrm{SO}(3)$, which here are different rotations of the Earth. At any instant in time $t$, the derivative of this path corresponds to a Lie algebra element $X(t) \in \mathfrak{s o}(3)$, which defines an infinitesimal isometry of $M$. However, $X(t)$ is just an anti-symmetric matrix (Fig. 5, bottom). The specific corresponding infinitesimal isometry of the sphere is given by an isomorphism between $\mathfrak{s o}(3)$ and the Lie algebra of Killing vector fields on the sphere (Fig. 5, top). This isomorphism is mathematically described by a Lie algebra action (see App. J). In this way, the infinitesimal isometry on the surface of the Earth is the Killing vector field $\mathbf{x}(t)$ corresponding to $X(t)$. At each point $x \in M$, this gives a relative velocity vector $\mathbf{x}(x, t)$ (Fig. 5, top). We also know that $\mathbf{x}$ describes an infinitesimal isometry of $M$, because $\nabla \mathbf{x}$, as the covariant derivative of $\mathbf{x}$, is anti-symmetric. ${ }^{2}$ In fact, any Killing field $\mathbf{x}$ is uniquely determined by its value $\mathbf{x}(x)$ and covariant derivative $(\nabla \mathbf{x})_{x}$ at one point $x \in M$ [53, Proposition 8.1.4].

\subsection{Observer Fields}

Generalizing further from the concept of one observer, as one timedependent isometric transformation of space, we want to be able to describe an observer field - many observers-on a manifold $M$ as a vector field $\mathbf{u}$ on $M$, where $\mathbf{u}$ does not necessarily correspond to an exact (infinitesimal) isometry. One of the most important properties of

${ }^{1} \mathrm{~A}$ (Riemannian) metric $\mathbf{g}$ is required to define the meaning of isometry.

${ }^{2}$ It is important to note, however, that checking $\nabla \mathbf{x}$ for anti-symmetry needs to be done with care, because it is a tensor of mixed type. See Sec. 7.1.

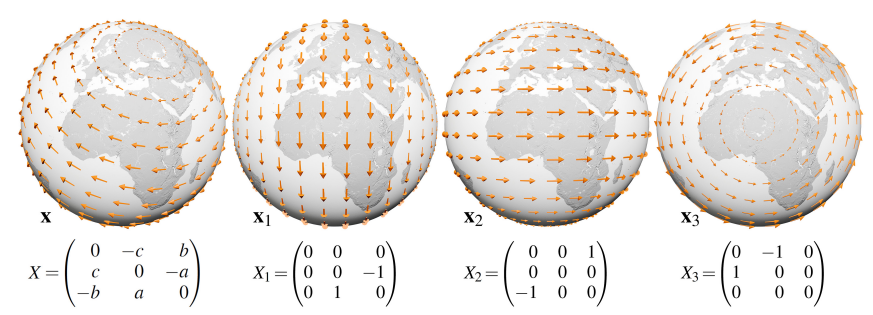

Fig. 5. Killing vector fields give the infinitesimal isometries of a manifold $M$, here the sphere $\mathbb{S}^{2}$. The Lie algebra elements $X, X_{i} \in \mathfrak{s o}(3)$ (bottom) generate the Killing fields $\mathbf{x}, \mathbf{x}_{i}$ on $M$ (top) through their Lie algebra action. Like the matrices $X_{i}$, the Killing fields $\mathbf{x}_{i}$ are linearly independent, forming a basis of the Lie algebra of Killing fields on $\mathbb{S}^{2}$. That is, as we can expand $X=a X_{1}+b X_{2}+c X_{3}$, we likewise get $\mathbf{x}=a \mathbf{x}_{1}+b \mathbf{x}_{2}+c \mathbf{x}_{3}$, where the latter means point-wise addition of vectors in each $T_{x} M$ at each $x \in M$.

such an observer field $\mathbf{u}$ is that we can compute it such that it minimizes the observed time derivative $(\mathscr{D} / \mathscr{D} t)(\mathbf{v}-\mathbf{u})$, given an arbitrary input vector field $\mathbf{v}$, whose time-dependent behavior we want to "follow."

The second important property of an observer field $\mathbf{u}$ is that if $\mathbf{u}$ is indeed a Killing vector field, it in fact describes a single observer, as defined above. Typically, we want to optimize the vector field $\mathbf{u}$ such that it describes observers that are as similar as possible. We do this by quantifying how much (or how "fast," in terms of spatial distance) the observers differ from one global isometry, by computing the Killing energy of the vector field $\mathbf{u}$ over $M$. This is the integral

$$
\int_{M} E \mathbf{u} \mathrm{d} A
$$

The point-wise energy term $E \mathbf{u}$ is given by Eq. 29. Eq. 3 is a scalar measure for how much $\mathbf{u}$ differs from an infinitesimal isometry on $M$. See Hadwiger et al. [30] for a more detailed rationale in Euclidean space. In this work, we now generalize this concept to curved spaces.

\subsection{Feature-Relative Visualization}

Fig. 1 and Fig. 6 illustrate how the computation of an observer field on the surface of the Earth enables focusing a visualization on a feature such as a hurricane. In this example, the input field $\mathbf{v}(x, t)$ is given by a time-dependent global wind data set from the European Copernicus project. The top row of Fig. 6 shows path lines following the motion of the hurricane over time. In this frame of reference, it is hard to compare features of the hurricane "in place," due to its motion. In contrast, the bottom row of Fig. 6 shows a visualization that is relative to an automatically computed observer field $\mathbf{u}(x, t)$, following the motion of the hurricane. Since now a "feature-centric" observer is used, the hurricane has become steady. (Now the Earth is moving, relative to the new observer, underneath the hurricane.) Path lines seeded at the same positions as before now clearly highlight the vortex of the hurricane.

\section{Objectivity in Curved Spaces}

Given a particular observer transformation $t \mapsto g(t) \in G$, it is natural to define that a tensor field is objective if it simply undergoes the same transformation as the observer. Because we model all possible observer transformations as paths through a continuous symmetry group $G$, i.e., a Lie group, we can now rigorously formulate a generalized concept of objectivity for arbitrary manifolds $M$ in a fully intrinsic manner, using the concept of group action on $M$ with a Lie group element $g \in G$.

We first summarize the definition of Truesdell and Noll [65], which is standard in continuum mechanics $[34,48]$, but is restricted to Euclidean space. We then introduce our completely general definition that includes curved spaces, but is equivalent in Euclidean space. App. D derives the definition of Truesdell and Noll more generally, illuminating the correspondence between their approach and ours in more detail.

\subsection{Objectivity in Euclidean space}

Truesdell and Noll [65, p.41] define objectivity with respect to a change of reference frame in $\mathbb{R}^{3}$ via the distance-preserving transformation

$$
\begin{aligned}
\mathbf{x}^{*} & =\mathbf{c}(t)+\mathbf{Q}(t) \mathbf{x}, \\
t^{*} & =t-a .
\end{aligned}
$$



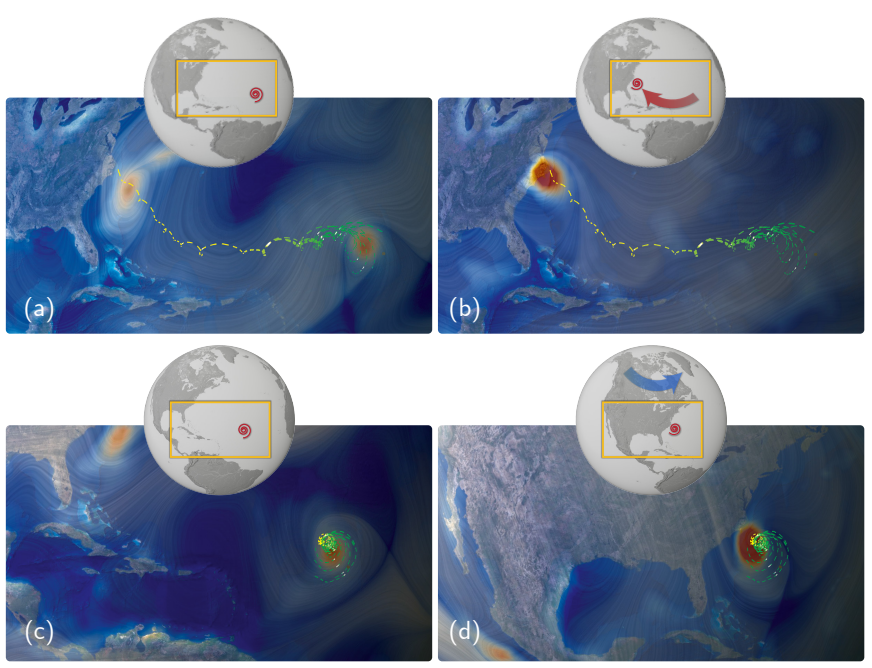

Fig. 6. Feature-centric visualization of hurricane Isabel. (a,b) Pathlines in the original flow field. (c,d) Observer-relative pathlines; the hurricane appears steady. (a,c) First time step. (b,d) Last time step. From (c) to (d), the Earth has moved underneath the steady hurricane.

$\mathbf{Q}(t)$ is a proper orthogonal tensor (a rotation), $\mathbf{c}(t)$ is a point (position vector), and $a \in \mathbb{R}$. This transformation assumes absolute time. It is thus sufficient to consider $a=0$, disregarding time shifts, giving $t^{*}=t$.

With respect to this transformation, a scalar field is objective if it is unchanged; a vector field $\mathbf{v}$ is objective if it transforms according to $\mathbf{v}^{*}=\mathbf{Q}(t) \mathbf{v}$; a second-order tensor field $\mathbf{S}$, as a linear transformation of vectors, is objective if it transforms as $\mathbf{S}^{*}=\mathbf{Q}(t) \mathbf{S} \mathbf{Q}(t)^{T}$ [65, p.42].

This entire definition depends on the domain being Euclidean: points are position vectors; the difference between two points is a vector; all tangent spaces are copies of $\mathbb{R}^{3}$ with trivial parallel transport. This definition is therefore not valid for non-Euclidean (curved) manifolds.

\subsection{Generalization of Objectivity}

To generalize objectivity, we define this concept as a general notion of tensor fields being invariant with respect to a continuous symmetry group $G$, which is a Lie group. (Symmetry refers to a notion of being the same.) For example, if the group $G$ is chosen as the isometry group of a (Riemannian) manifold, two tensor fields are "the same" if they are isometric. Two fields being symmetries of each other then means that there exists a group element $g \in G$, such that the transformation rules given below hold. Then, given any time-dependent observer transformation $t \mapsto g(t) \in G$, a given tensor field is objective if, for each fixed $t$, it simply follows the corresponding transformation $g:=g(t)$.

\subsubsection{Symmetry groups and group actions}

Our notion of symmetry corresponds to the transformation behavior under a group action $\Phi$, with a given Lie group element $g \in G$, where $G$ is the chosen symmetry group. An action $\Phi$, specifically a smooth left action, of a Lie group $G$ on a manifold $M$, is a smooth map [33, p.209]

$$
\begin{aligned}
\Phi: G \times M & \rightarrow M, \\
(g, x) & \mapsto \Phi(g, x),
\end{aligned}
$$

such that for every $g \in G$, the map

$$
\phi_{g}: M \rightarrow M, \text { with } \phi_{g}(x):=\Phi(g, x), \text { is a diffeomorphism. }
$$

Here, we focus on the general use of group actions $\Phi$ in our context, and defer details to later sections. For now, it is sufficient to understand that the diffeomorphisms $\phi_{g}$ will correspond to the flows of specific vector fields on $M$. These vector fields are generated by the action of the Lie algebra $\mathfrak{g}$ of the Lie group $G$ on $M$. See App. J for details.

For example, if $G$ is the group of all diffeomorphisms of $M$, these vector fields are all possible (smooth) vector fields on $M$. The important case for our framework is choosing the group $G$ as the isometry group of $M$. The corresponding vector fields are then the Killing vector fields on $M$, whose flows correspond to the isometries of $M$. See Sec. 7.
To obtain a generalized definition of objectivity, a crucial property of the diffeomorphism $\phi_{g}$ is that it enables us to use the corresponding differential, or pushforward. See Fig. 7. The pushforward is a map

$$
\mathrm{d} \phi_{g}: T M \rightarrow T M
$$

where each $\left(\mathrm{d} \phi_{g}\right)_{x}$ at a point $x \in M$ is a linear map

$$
\begin{aligned}
\left(\mathrm{d} \phi_{g}\right)_{x}: T_{x} M & \rightarrow T_{\phi_{g}(x)} M, \\
\mathbf{v} & \mapsto\left(\mathrm{d} \phi_{g}\right)_{x}(\mathbf{v}) .
\end{aligned}
$$

The notation $(\cdot)_{x}$ means that the quantity in parentheses is located at $x \in M$, and $T_{x} M$ denotes the tangent space at $x$. We can simply imagine that the diffeomorphism $\phi_{g}$ transforms curves on $M$, and the differential $\mathrm{d} \phi_{g}$ transforms their tangent vectors accordingly. See also App. U.

In components, the map $\left(\mathrm{d} \phi_{g}\right)_{x}$ at any $x \in M$ can be given by the corresponding $n \times n$ matrix. See Fig. 7 for the case of a sphere $(n=2)$.

Euclidean space. When $\phi_{g}$ is an isometry of $M=\mathbb{R}^{3}$, the pushforward $\mathrm{d} \phi_{g}$ is a globally constant proper orthogonal (rotation) tensor $\mathbf{Q}$, i.e., $\left(\mathrm{d} \phi_{g}\right)_{x}=\mathbf{Q}$, with the same $\mathbf{Q}$ at all $x \in M$. See O'Neill [49, p.107].

Curved spaces. In general, however, the linear map $\left(\mathrm{d} \phi_{g}\right)_{x}$ is different for different points $x \in M$. In components, each $\left(\mathrm{d} \phi_{g}\right)_{x}$ can still be given by a matrix, but it will be a different matrix for each point $x \in M$.

\subsubsection{Objective scalar fields}

Being objective should mean invariant under transformation, which for scalar fields is trivial. We therefore define that a scalar field $f: M \rightarrow \mathbb{R}$ on a manifold $M$ is objective when, under any diffeomorphism $\phi_{g}$, given by the group action $\Phi$ of a symmetry group $G$, it transforms as

$$
f^{*}\left(\phi_{g}(x)\right)=f(x) \text {. }
$$

Abbreviated, we could write $f^{*}=f$, but it is crucial to note that $f^{*}$ is evaluated at the point $\phi_{g}(x)$, whereas $f$ is evaluated at the point $x$.

\subsubsection{Objective vector fields}

We now define that an arbitrary vector field $\mathbf{v}$ on a manifold $M$ is objective (with respect to a given symmetry group $G$ ), if, under the corresponding group action $\Phi$ with any $g \in G$, it transforms as

$$
\left(\mathbf{v}^{*}\right)_{\phi_{g}(x)}=\left(\mathrm{d} \phi_{g}\right)_{x}(\mathbf{v}) .
$$

We emphasize that $\mathbf{v}^{*}$ is an element of the tangent space $T_{\phi_{g}(x)} M$, whereas $\mathbf{v}$ is an element of $T_{x} M$. Likewise, it is important to note that the differential $\left(\mathrm{d} \phi_{g}\right)_{x}$ is a linear map defined on $T_{x} M$. We can say

Remark. A vector field is objective, if it is simply pushed forward by any diffeomorphism $\phi_{g}$, defined according to the group action $\Phi$. This definition of objectivity is valid for any smooth manifold where a notion of (smooth) symmetry is defined by a (smooth) symmetry group $G$.

Abbreviated transformation rule. For brevity, we can define the action $\Phi$, with $g \in G$, on any vector field $\mathbf{v}$ on $M$, by the differential in Eq. 8, and abbreviate the objectivity criterion of Eq. 10 simply as

$$
\mathbf{v}^{*}=g \mathbf{v}
$$

However, it is crucial that the meaning of the transformation represented by $g \in G$ in this shorthand notation is given by Eq. 10. In general, $g$ cannot be mapped to the same globally defined matrix, corresponding to the pushforward $\mathrm{d} \phi_{g}$, even though this is possible in the Euclidean case. Nevertheless, this abbreviated form makes it easy to see the analogy with the definition of Truesdell and Noll. In Euclidean space, the two are equivalent. See App. D for more details. Our definition, however, gives a well-defined notion of objectivity for arbitrary manifolds $M$. 


\subsubsection{Objective tensor fields}

The definition above for objective vectors generalizes to arbitrary tensor fields $\mathbf{T}$, in the way that is derived in full in App. A. Here, we derive the common special case of a second-order tensor field $\mathbf{S}$, interpreted as a linear map of vectors, i.e., $\mathbf{S}: T M \rightarrow T M, \mathbf{v} \mapsto \mathbf{S}(\mathbf{v})$. Such a $\left(\begin{array}{l}1 \\ 1\end{array}\right)$ tensor field is objective, if under the group action $\Phi$ with $g$ it transforms as

$$
\left(\mathbf{S}^{*}\right)_{\phi_{g}(x)}\left(\mathbf{v}^{*}\right)=\left(\mathrm{d} \phi_{g}\right)_{x}\left(\mathbf{S}\left(\left(\mathrm{d} \phi_{g^{-1}}\right)_{\phi_{g}(x)}\left(\mathbf{v}^{*}\right)\right)\right) .
$$

The pushforward $\mathrm{d} \phi_{g^{-1}}$ of the diffeomorphism $\phi_{g^{-1}}$ maps $\mathbf{v}^{*}$ from $T_{\phi_{g}(x)} M$ to the corresponding $\mathbf{v}$ in $T_{x} M$ according to Eq. 10 (inverted) and then applies Eq. 10 again to $\mathbf{S}(\mathbf{v}) . \phi_{g^{-1}}$ is the inverse of $\phi_{g}$, meaning $\phi_{g^{-1}}\left(\phi_{g}(x)\right)=\phi_{g^{-1} g}(x)=\phi_{e}(x)=x$. As in Eq. 11, we can abbreviate

$$
\mathbf{S}^{*}=g \mathbf{S} g^{-1} \text {. }
$$

However, it is again crucial to note that the meaning of this rule is given by Eq. 12. Fig. 7 illustrates how the pushforwards of $\phi_{g}$ and $\phi_{g^{-1}}$ map tangent vectors between the tangent spaces $T_{\phi_{g}(x)} M$ and $T_{x} M$.

We note that to prove objectivity of the Killing operator $K$ (Eq. 25), which we use for computing observer fields, the general definition of objectivity from App. A is required, since $K \mathbf{u}$ is a $\left(\begin{array}{l}0 \\ 2\end{array}\right)$ tensor (App. F.1).

\subsection{Objectivity for Intrinsic Isometries}

To realize the notion of observer transformations as isometric transformations of the underlying space in the general mathematical framework given above, we now consider the group $G:=\operatorname{Isom}(M)$, i.e., the isometry group of the manifold $M$. The group action $\Phi$ is an isometry, with respect to a given metric $\mathbf{g}$, i.e., a smoothly varying inner product $\langle\cdot, \cdot\rangle$, on $M$, if for all $g \in G$, and for any two vector fields $\mathbf{v}, \mathbf{w}$ on $M$, we have

$$
\left\langle\left(\mathrm{d} \phi_{g}\right)_{x}(\mathbf{v}),\left(\mathrm{d} \phi_{g}\right)_{x}(\mathbf{w})\right\rangle_{\phi_{g}(x)}=\langle\mathbf{v}, \mathbf{w}\rangle_{x} .
$$

As a direct consequence of Eq. 14 holding for the map $\phi_{g}$, we get that for any vector field $\mathbf{v}$ that is objective according to Eq. 10, we have

$$
\left\|\mathbf{v}^{*}\right\|_{\phi_{g}(x)}=\|\mathbf{v}\|_{x} \text {. }
$$

Infinitesimal isometries and Killing vector fields. Computationally, instead of working with isometries directly, we work with their derivatives, which are infinitesimal isometries, corresponding to the Killing fields on $M$. See Sec. 7. To describe Killing fields efficiently, we need the intrinsic velocity gradient tensor $\nabla \mathbf{v}$ of a velocity field $\mathbf{v}$ on $M$.

\section{General Velocity Gradient Tensors}

For any smooth vector field $\mathbf{v}$ given on a manifold $M$, the velocity gradient tensor $\nabla \mathbf{v}$ is a second-order tensor field on $M$ that allows computing directional derivatives of the vector field $\mathbf{v}$ in any direction.

In Cartesian coordinates, $\nabla \mathbf{v}$ can simply be defined by partial derivatives $\partial_{j} v^{i}$. However, this definition cannot be used for intrinsic computations on curved manifolds, because $\partial_{j} v^{i}$ is in general not a tensor. For a curved (or flat) manifold $M$, the tensor $\nabla \mathbf{v}$ can, however, always be computed intrinsically as the covariant derivative of $\mathbf{v}[22, \mathrm{p} .241]$.

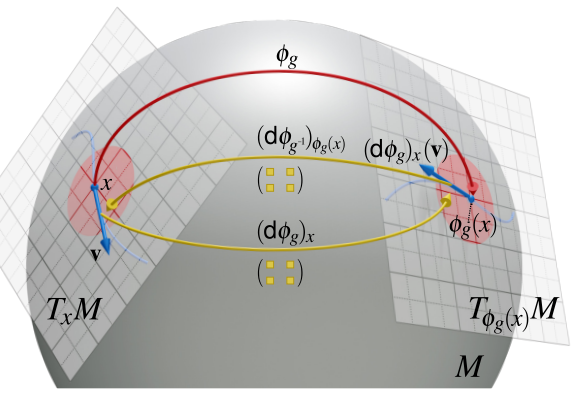

Fig. 7. The pushforward of a diffeomorphism $\phi_{g}$ is a linear map between the tangent spaces $T_{x} M$ and $T_{\phi_{g}(x)} M$. That is, the pushforward $\left(\mathrm{d} \phi_{g}\right)_{x}$ maps a tangent vector $\mathbf{v} \in T_{x} M$ to the vector $\left(\mathrm{d} \phi_{g}\right)_{x}(\mathbf{v}) \in T_{\phi_{g}(x)} M$. The pushforward $\left(\mathrm{d} \phi_{g^{-1}}\right)_{\phi_{g}(x)}$ maps a tangent vector in the opposite direction. In components, for a two-manifold $M$, each pushforward is a $2 \times 2$ matrix.

\subsection{Intrinsic Covariant Derivatives}

The covariant derivative $\nabla \mathbf{v}$ of a vector field $\mathbf{v}$ on a manifold $M$ is a second-order tensor field on $M$, which, at any $x \in M$, maps an arbitrary vector $\mathbf{x} \in T_{x} M$ to the derivative of the field $\mathbf{v}$ in the direction $\mathbf{x}$, i.e.,

$$
\begin{aligned}
(\nabla \mathbf{v})_{x}: T_{x} M & \rightarrow T_{x} M, \\
\mathbf{x} & \mapsto \nabla_{\mathbf{x}} \mathbf{v}=(\nabla \mathbf{v})_{x}(\mathbf{x}) .
\end{aligned}
$$

This notation means that the covariant derivative of a vector field $\mathbf{v}$, evaluated in the direction $\mathbf{x}$, is the vector $\nabla_{\mathbf{x}} \mathbf{v}$, which we get by computing $\nabla \mathbf{v}(\mathbf{x})$. We can refer $\nabla \mathbf{v}$ to a basis $\left\{\mathbf{e}_{i} \otimes \omega^{j}\right\}$, with $\otimes$ the tensor product, and write the components as $n \times n$ matrix $\nabla_{j} v^{i}$, with row and column indices $i, j$, respectively, and $n$ the dimension of $M$, i.e., for a curved surface $n=2$. However, unless the manifold $M$ is flat and Cartesian or affine coordinates are used, $\nabla_{j} v^{i} \neq \partial_{j} v^{i}$. The difference is a connection on $M$ [22, p.242], corresponding to a notion of parallel transport $[19, \mathrm{p} .52]$ of vectors. The connection can be given in components via Christoffel symbols $\Gamma_{j k}^{i}$, and we get for $\nabla \mathbf{v}$ in components,

$$
\nabla_{j} v^{i}:=\partial_{j} v^{i}+\Gamma_{j k}^{i} v^{k}
$$

In flat space with Cartesian or affine coordinates, all Christoffel symbols vanish [24, p.72] $\left(\Gamma^{i}{ }_{j k} \equiv 0\right)$, but only in this special case is $\nabla_{j} v^{i}=\partial_{j} v^{i}$. The $\Gamma^{i}{ }_{j k}$ determine the covariant derivatives of the basis vector fields $\left\{\mathbf{e}_{i}\right\}$, at each point $x \in M$, referred back to the same basis $\left\{\mathbf{e}_{i}\right\}$ at $x$, i.e.,

$$
\nabla_{\mathbf{e}_{j}} \mathbf{e}_{k}=\Gamma_{j k}^{i} \mathbf{e}_{i} \text {, and therefore } \Gamma^{i}{ }_{j k}=\omega^{i}\left(\nabla_{\mathbf{e}_{j}} \mathbf{e}_{k}\right) \text {. }
$$

We note that although $\Gamma_{j k}^{i}$ comprises $n^{3}$ components, it is not a (thirdorder) tensor [24, p.82]. In general, a connection needs to be chosen [19, p.50]. We employ the Levi-Civita connection, ${ }^{3}$ which can be derived intrinsically from the metric of $M$ [22, p.229]. See App. O, Eq. O.10. Even more easily, if we know an immersion of $M$ into an ambient space $\mathbb{R}^{m}$, as for a curved surface immersed in $\mathbb{R}^{3}$, we can obtain the $\Gamma^{i}{ }_{j k}$ as

$$
\Gamma_{j k}^{i}=\tilde{\omega}^{i}\left(\partial_{j} \tilde{\mathbf{e}}_{k}\right)
$$

The tilde symbols denote ambient space (co)vectors, e.g., for $M$ with $n=2$ and ambient $m=3,\left\{\partial_{j} \tilde{\mathbf{e}}_{k}\right\}$ comprises four $3 \mathrm{D}$ vectors, and $\left\{\tilde{\omega}^{i}\right\}$ comprises two 3D covectors. It is crucial that the $\left\{\tilde{\omega}^{i}\right\}$ are chosen such that they correspond to orthogonal projection from $\mathbb{R}^{m}$ onto the tangent plane of the surface. See App. Q for a complete calculation for a simple atlas of charts for the sphere. The Levi-Civita connection given by this choice of $\Gamma_{j k}^{i}$ corresponds to the metric on $M$ that is induced by the immersion of $M$ into the ambient space $\mathbb{R}^{m}[19$, p.39]. Crucially, however, the resulting Christoffel symbols are nevertheless intrinsic.

\section{Isometries and Killing Fields in CuRved Spaces}

Our framework requires an efficient mathematical description of the intrinsic isometries of the manifold $M$. A powerful way to do this is to work with the derivatives of diffeomorphisms that are isometries, i.e., to consider infinitesimal isometries. These correspond to specific vector fields on the manifold $M$, which are called Killing vector fields.

A vector field $\mathbf{u}$ is a Killing vector field on $M$, if the flow $\phi_{t}$ (App. T) that it generates preserves the metric $\mathbf{g}$ on $M$. The most general way of stating this mathematically is to require that the Lie derivative (App. G) of the metric $\mathbf{g}$, with respect to $\mathbf{u}$, must vanish everywhere on $M$. I.e,

$$
\mathscr{L}_{\mathbf{u}} \mathbf{g}=0 .
$$

This means that for all $x \in M$, we have $\left(\mathscr{L}_{\mathbf{u}} \mathbf{g}\right)_{x}=0$. See [46, p.250].

The set of all possible Killing vector fields on a given (Riemannian) manifold $M$ constitutes a Lie algebra, which is, in particular, a vector space. This enables us to consider the dimensionality of the set of

${ }^{3}$ This is the unique metric-compatible connection, corresponding to a given (Riemannian) metric $\mathbf{g}$ on the manifold $M$, that has zero torsion [22, p.242]. 

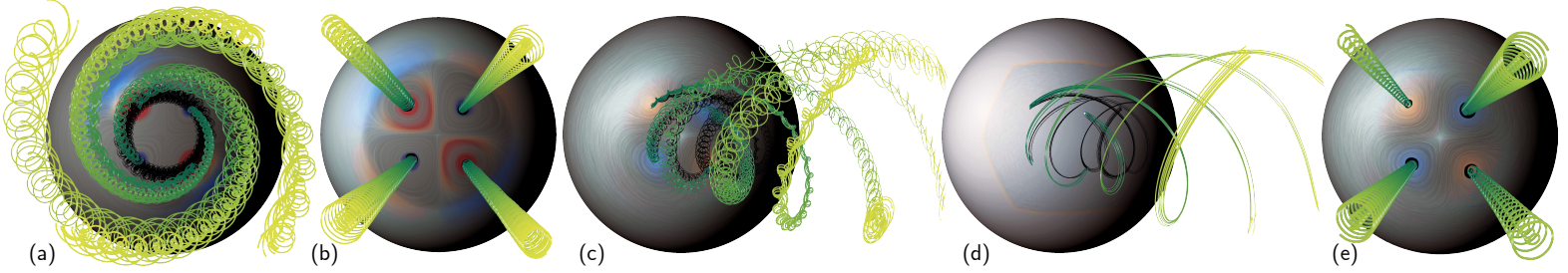

Fig. 8. Rotating four centers relative to three different observers. Path lines seeded close to the four centers are shown in space-time as in Fig. 3. (a) Observer rotating counter-clockwise around the center point between the four vortices. (b) The observer rotation in (a) is automatically removed relative to an optimized observer field $\mathbf{u}$, which corresponds to clockwise rotation: the four centers appear steady. (c) The field in (a) relative to an observer rotating around the vertical axis. (d) The observer field $\mathbf{u}$ (path lines) optimized for the field in (c). (e) The field in (c) relative to $\mathbf{u}$ from (d).

Killing fields on $M$, and to write any Killing field as a linear combination of basis Killing fields. Fig. 5 shows an example on the sphere. Apps. $\mathrm{L}$ and $\mathrm{M}$ describe the isometries of the two-sphere in detail, with full calculations. App. K gives more details on Killing vector fields on curved surfaces in general. However, for computing observer fields via optimization (Sec. 9), as the most important part of our framework, we directly evaluate Killing's equation on the unknown vector field $\mathbf{u}$.

\subsection{Killing's Equation}

A vector field $\mathbf{u}=u^{i} \mathbf{e}_{i}$ is a Killing field, if an equation known as Killing's equation holds at all $x \in M$ [22, p.529]. However, in its general form this equation is given for covectors, with components $u_{i}$, and not for vectors, with components $u^{i}$. The $u_{i}$ can be computed as $u_{i}=g_{i j} u^{j}[24, \mathrm{p} .88]$, using a metric $\mathbf{g}$, referred to components $g_{i j}$.

Killing's equation is then given as

$$
\nabla_{j} u_{i}+\nabla_{i} u_{j}=0
$$

This is the same as $\nabla \mathbf{u}$ being anti-symmetric, which is the same as $\langle\nabla \mathbf{u}(\mathbf{x}), \mathbf{x}\rangle=0$ for all $\mathbf{x}$. However, in general it is not correct to simply check whether a component matrix of $\nabla \mathbf{u}$ is anti-symmetric, because $\nabla \mathbf{u}$ is a tensor of mixed type (contravariant and covariant indices). Therefore, Eq. 21 is given using the covariant derivative of a covector field [24, p.108]. In components, referred to a basis $\left\{\omega^{i} \otimes \omega^{j}\right\}$, this is

$$
\nabla_{j} u_{i}:=\partial_{j} u_{i}-\Gamma_{i j}^{k} u_{k}
$$

We can introduce the vector components $u^{i}$ directly, and write Eq. 21 as

$$
\nabla_{j}\left(g_{i k} u^{k}\right)+\nabla_{i}\left(g_{j k} u^{k}\right)=0
$$

Applying the product rule for covariant derivatives [24, p.119], we get $\nabla_{j}\left(g_{i k} u^{k}\right)=\left(\nabla_{j} g_{i k}\right) u^{k}+g_{i k} \nabla_{j} u^{k}=g_{i k} \nabla_{j} u^{k}$, because the covariant derivative of the metric vanishes. ${ }^{4}$ Therefore, Eq. 21 is equivalent to

$$
g_{i k} \nabla_{j} u^{k}+g_{j k} \nabla_{i} u^{k}=0
$$

In matrix notation, we can therefore rewrite Killing's equation as the vanishing of the differential "Killing operator" $K$ applied to the field $\mathbf{u}$,

$$
K \mathbf{u}:=\mathbf{g} \nabla \mathbf{u}+(\mathbf{g} \nabla \mathbf{u})^{T}=0
$$

The tensor $K \mathbf{u}$ is still a $\left(\begin{array}{l}0 \\ 2\end{array}\right)$ tensor, just like $\left(\nabla_{j} u_{i}+\nabla_{i} u_{j}\right)$. However, Eq. 25 implies that now we can simply check any matrix representation of $\mathbf{g} \nabla \mathbf{u}$ for anti-symmetry, to determine whether $\mathbf{u}$ is a Killing field.

\subsection{Killing Energy}

If the expression $K \mathbf{u}$ is not exactly zero, in order to quantify how close to being Killing an arbitrary vector field $\mathbf{u}$ is, we use the tensor norm of the tensor Ku to define a scalar Killing energy (see also [9, Def. 5]). As for vectors, the tensor norm of a higher-order tensor $\mathbf{T}$ is defined as

$$
\|\mathbf{T}\|_{\mathbf{g}}^{2}:=\langle\mathbf{T}, \mathbf{T}\rangle_{\mathbf{g}}
$$

However, the tensor inner product required here, between two covariant second-order tensors $\mathbf{T}, \mathbf{S}$, is given by the double contraction [45, p.82]

$$
\begin{gathered}
\langle\mathbf{T}, \mathbf{S}\rangle_{\mathbf{g}}:=g^{i k} g^{j l} T_{k l} S_{i j}=T^{i j} S_{i j} \\
{ }^{4} \text { This }(\nabla \mathbf{g}=0) \text { is the definition of a metric connection } \\
\text { [22, p.242]. }
\end{gathered}
$$

This is similar to the Frobenius inner product $\operatorname{tr}\left(\mathbf{T}^{T} \mathbf{S}\right)$ of matrices, which determines the Frobenius norm $\operatorname{tr}\left(\mathbf{T}^{T} \mathbf{T}\right)^{1 / 2}$. The crucial difference here is that because pairs of covariant and contravariant indices are contracted, Eq. 27 is an invariant tensor expression, giving the same result in all coordinate systems. $\langle\mathbf{T}, \mathbf{T}\rangle_{\mathbf{g}}=T^{i j} T_{i j}$ thus is the square of the norm. In matrix notation, we can write the inner product above as

$$
\langle\mathbf{T}, \mathbf{S}\rangle_{\mathbf{g}}=\operatorname{tr}\left(\mathbf{g}^{-1} \mathbf{T}^{T} \mathbf{g}^{-1} \mathbf{S}\right)
$$

We define the Killing energy $E \mathbf{u}$, of the field $\mathbf{u}$, at a point $x \in M$, as

$$
E \mathbf{u}:=\|K \mathbf{u}\|_{\mathbf{g}}^{2}=\langle K \mathbf{u}, K \mathbf{u}\rangle_{\mathbf{g}} .
$$

Sec. 9.1 defines the Killing energy of $M$ as the integral of $E \mathbf{u}$ over $M$. Eq. 29 is then the density of the Killing energy of $M$ per unit area.

\section{Observed Time Derivatives in Curved Spaces}

The observed time derivative defined in Eq. 1 measures the differential change of a tensor field with respect to the motion of a given observer field $\mathbf{u}$. We note that $\mathscr{D} / \mathscr{D} t$ is, in fact, the time-dependent Lie derivative [45, p.95] with respect to the flow of the field $\mathbf{u}$. The crucial difference here is the semantic meaning of an observer field $\mathbf{u}$, as an intrinsic part of our operator. Our most important use of $\mathscr{D} / \mathscr{D} t$ is applying it to the relative velocity field $(\mathbf{v}-\mathbf{u})$, which we minimize to compute the unknown observer field $\mathbf{u}(\operatorname{Sec} .9)$. We get $\left(\mathscr{L}_{\mathbf{u}} \mathbf{u}=0\right)$,

$$
\begin{aligned}
\frac{\mathscr{D}}{\mathscr{D} t}(\mathbf{v}-\mathbf{u}) & =\frac{\partial \mathbf{v}}{\partial t}-\frac{\partial \mathbf{u}}{\partial t}+\mathscr{L}_{\mathbf{u}} \mathbf{v} \\
& =\frac{\partial \mathbf{v}}{\partial t}-\frac{\partial \mathbf{u}}{\partial t}+\nabla \mathbf{v}(\mathbf{u})-\nabla \mathbf{u}(\mathbf{v})
\end{aligned}
$$

Lie derivatives in curved spaces. A basic property of Lie derivatives is that they are independent of the metric $\mathbf{g}$ defined on the manifold $M$, and likewise independent of the connection on $M[45, \mathrm{p} .96]$. That is,

$$
\begin{aligned}
\mathscr{L}_{\mathbf{u}} \mathbf{v} & =\nabla \mathbf{v}(\mathbf{u})-\nabla \mathbf{u}(\mathbf{v}), \\
& =\left(\partial_{j} v^{i} u^{j}-\partial_{j} u^{i} v^{j}\right) \mathbf{e}_{i} .
\end{aligned}
$$

The expansion in components in the second row above comprises only partial derivatives. The full derivation is given in App. H. Thus, the observed time derivative can be derived solely from partial derivatives, even when the manifold $M$ is curved, including the $\mathscr{L}_{\mathbf{u}}$ term in Eq. 30 .

\section{Computing Observer Fields}

We compute a global observer field $\mathbf{u}$ on any two-manifold $M$ with multiple charts using one global linear least-squares solve. The use of a single global sparse linear system is enabled by "baking" all transition maps (their Jacobian matrices) between charts into the system matrix.

\subsection{Objective Function}

Our optimization is conceptually very similar to the approach of Hadwiger et al. [30] for Euclidean space. However, all differential operators have to be substituted by their generalized version for arbitrary manifolds, i.e., covariant derivatives, Lie derivatives, and tensor norms. 
We formulate a global optimization problem to find an observer field $\mathbf{u}$, from the space of all possible fields in some function space $\mathscr{V}$ of vector fields (e.g., $C^{n}$ fields) on the manifold $M$, as the minimizer of

$$
\min _{\mathbf{u} \in \mathscr{V}} \frac{1}{2} \int_{t_{\min }}^{t_{\max }} \int_{M}(E+\lambda D+\mu R)(\mathbf{u}, x, t) \mathrm{d} A \mathrm{~d} t .
$$

Here, $\mathrm{d} A$ is an infinitesimal area element of $M$ at a point $x \in M$, and the constants $\lambda, \mu \in \mathbb{R}$ are relative weights. The individual terms are

$$
\begin{aligned}
& E(\mathbf{u}, x, t):=\|K \mathbf{u}(x, t)\|_{\mathbf{g}}^{2}, \\
& D(\mathbf{u}, x, t):=\left\|\frac{\mathscr{D}}{\mathscr{D} t}(\mathbf{v}-\mathbf{u})(x, t)\right\|_{\mathbf{g}}^{2}, \\
& R(\mathbf{u}, x, t):=\|(\mathbf{v}-\mathbf{u})(x, t)\|_{\mathbf{g}}^{2} .
\end{aligned}
$$

The integral of $E$ over the domain gives the Killing energy $\int_{M} E \mathbf{u} \mathrm{d} A$ of the observer field $\mathbf{u}$ on $M .\|\cdot\|_{\mathrm{g}}$ denotes the tensor and vector norms, respectively, depending on the argument, with respect to the metric $\mathbf{g}$.

Discretization. To solve Eq. 32, we discretize the manifold $M$ with a triangle mesh. Vector components are stored at the vertices, and we label the corresponding points on the manifold $M$ with $x_{1}, x_{2}, \ldots, x_{N}$.

The Killing energy term $E$ (Eq. 33) is computed by discretizing the Killing operator $K$ (Eq. 25), to compute the covariant derivative terms of $\nabla \mathbf{u}$ in Eq. 25. The weights for all differential operators to evaluate $K \mathbf{u}$ are stored in a matrix $\mathbf{K}$. We compute partial derivatives via triangle vertex 1-ring neighborhood stencils, computing weighted averages of function values at the mesh vertices. The weights are independent of the actual function values, and can therefore by pre-computed for a fixed mesh geometry and be used for any function on $M$. See App. R.

The observed time derivative term $D$ (Eq. 34) is computed from partial derivatives alone (Eqs. 30 and 31). We compute time derivatives via central differences (in $t$ ) on each vertex. The weights for all differential operators to compute $D$ are stored in a matrix $\mathbf{D}$. The regularization term $R$ (Eq. 35) is represented by a matrix $\mathbf{N}$, which is simply an identity matrix times -1 , to compute $-\mathbf{u}$. All terms involving the (known) input field $\mathbf{v}$ are pre-computed and stored in the corresponding locations of the matrices $\mathbf{K}, \mathbf{D}$, and the right-hand side $\mathbf{b}$ (see below).

\subsection{Global Least-Squares Solution}

To solve Eq. 32, we are looking for the minimizer of the least-squares problem $\min _{\mathbf{u}} \frac{1}{2}\|\mathbf{A u}-\mathbf{b}\| \|_{\mathbf{g}}^{2}$, with matrix $\mathbf{A}$ and vector $\mathbf{b}$ as given below. The least-squares solution, weighted with the metric $\mathbf{g}$, is the solution of the normal equations of the weighted least-squares problem given by the square, positive-definite system $\mathbf{A}^{T} \mathbf{W} \mathbf{A} \mathbf{u}=\mathbf{A}^{T} \mathbf{W} \mathbf{b}$, with

$$
\mathbf{A}:=\left(\begin{array}{c}
\mathbf{K} \\
\mathbf{D} \\
\mathbf{N}
\end{array}\right), \mathbf{W}:=\mathbf{M} \cdot\left(\begin{array}{ccc}
\overline{\mathbf{G}} & 0 & 0 \\
0 & \lambda \cdot \mathbf{G} & 0 \\
0 & 0 & \mu \cdot \mathbf{G}
\end{array}\right), \mathbf{b}:=-\left(\begin{array}{c}
0 \\
\frac{\partial \mathbf{v}}{\partial t} \\
\mathbf{v}
\end{array}\right) .
$$

The matrix $\mathbf{M}$ is diagonal, with weights giving the area on $M$ assigned to each vertex element $x_{i}$, discretizing integration over area elements $\mathrm{d} A$. The other blocks of the matrix $\mathbf{W}$ encode $\mathbf{g}$ on $M$, given by the blocks

$$
\overline{\mathbf{G}}:=\left(\begin{array}{cccc}
\overline{\mathbf{g}}_{x_{1}}^{-1} & 0 & \ldots & 0 \\
0 & \overline{\mathbf{g}}_{x_{2}}^{-1} & \ldots & 0 \\
\vdots & \vdots & \ddots & \vdots \\
0 & 0 & \ldots & \overline{\mathbf{g}}_{x_{N}}^{-1}
\end{array}\right), \mathbf{G}:=\left(\begin{array}{cccc}
\mathbf{g}_{x_{1}} & 0 & \ldots & 0 \\
0 & \mathbf{g}_{x_{2}} & \ldots & 0 \\
\vdots & \vdots & \ddots & \vdots \\
0 & 0 & \ldots & \mathbf{g}_{x_{N}}
\end{array}\right) .
$$

Here, $\mathbf{g}_{x_{i}}$ refers to the $n \times n$ component matrix $g_{i j}$ of the metric $\mathbf{g}$ at the vertex $x_{i} \in M$. For efficient computation of the tensor norm $\|\mathbf{T}\|_{\mathbf{g}}$, we use the following approach to convert the tensor inner product in Eq. 27 to a simple contraction of two first-order tensors. We introduce new $\bar{n}$-dimensional first-order tensors $\overline{\mathbf{T}}, \overline{\mathbf{S}}$, and a symmetric $\bar{n}$-dimensional second-order tensor $\overline{\mathbf{g}}^{-1}$, where $\bar{n}:=n^{2}$. That is, $\bar{n}=4$ for $n=2$. We denote the $\bar{n} \times \bar{n}$ components of $\overline{\mathbf{g}}^{-1}$ by $\bar{g}^{s t}$, computed from $g^{i j}$ as

$$
\bar{g}^{s t}:=g^{i k} g^{j l}, \quad s, t \in[1, \bar{n}], \quad(i, j)=\sigma^{-1}(s),(k, l)=\sigma^{-1}(t) .
$$

The indices $i, j, k, l$ are given by the inverse of the indexing function

$$
\sigma(i, j):=n \cdot(i-1)+j, \text { with } i, j \in[1, n] .
$$

We can use this to rearrange the tensor inner product, to obtain

$$
\begin{array}{rlrl}
\bar{T}_{t} & :=T_{k l}, & \text { with } t=\sigma(k, l), \\
\bar{S}_{s} & :=S_{i j}, & \text { with } s=\sigma(i, j), \\
\bar{T}^{s} & =\bar{g}^{s t} \bar{T}_{t}, & \text { and therefore, } \\
\bar{T}^{s} \bar{S}_{s} & =\bar{g}^{s t} \bar{T}_{t} \bar{S}_{s}=g^{i k} g^{j l} T_{k l} S_{i j}=T^{i j} S_{i j} .
\end{array}
$$

Thus, the tensor inner product $\langle\mathbf{T}, \mathbf{S}\rangle_{\mathbf{g}}$ (Eq. 27) can simply be computed as the contraction $\bar{T}^{s} \bar{S}_{s}$. Using this approach, the tensor norm of the Killing energy in the least-squares problem given above is computed by inserting the components $\bar{g}^{s t}$ (Eq. 38) of $\overline{\mathbf{g}}^{-1}$ into the matrix $\overline{\mathbf{G}}$ above.

\subsection{Multi-Chart Optimization}

At each vertex $x_{i} \in M$, there is only one vector for the field $\mathbf{u}$. We store vector components with respect to one selected chart for each $x_{i}$. However, finite differences to estimate derivatives (App. R) have to be computed in the same chart, even when adjacent vertices are in different charts. We enable this by "baking" all required chart transition maps, i.e., the $n \times n$ Jacobian matrices transforming from the coordinate basis of one chart to that of another chart, into the matrices $\mathbf{K}$ and $\mathbf{D}$. The elements of these matrices are inserted such that all finite differences are computed using vector components referencing the same chart. More details for implementing this approach are explained in App. S.

\subsection{Objectivity of Intrinsic Observer Field Computation}

Remark. The computation of the observer field $\mathbf{u}$ as the minimizer of Eq. 32 is objective with respect to any observer transformation $t \mapsto g(t) \in G$, with $G=\operatorname{Isom}(M)$ the isometry group of the manifold $M$.

We give proofs in App. F. Except point (4) below, the reasons for this are quite intuitive and can be summarized as follows: (1) The norms used in Eq. 32 are invariant under isometries, because by definition any isometry does not change them (Eq. 14). (2) The Killing energy $\int_{M} E \mathbf{u} \mathrm{d} A$ is time-independent, and measures how much the field $\mathbf{u}$ differs from an infinitesimal isometry. Since observer transformations are exact isometries of $M$, they do not change the Killing energy. (3) A timedependent observer transformation $t \mapsto g(t)$, which with $G=\operatorname{Isom}(M)$ is a time-dependent isometry $t \mapsto \phi_{g(t)}$ of $M$, changes any velocity field on $M$ by point-wise addition of the Killing field corresponding to the derivative $X(t)$ of the isometry. However, the Killing field that is added due to the observer transformation cancels out in the relative velocity field $(\mathbf{v}-\mathbf{u})$. (4) The objectivity of the observed time derivative (Eq. 1) is far from obvious. However, Marsden and Hughes [45, p.101] prove that the time-dependent Lie derivative of an objective tensor field with respect to the flow of any vector field is objective. See App. F.3.

That is, the norm of all terms in Eq. 32 does not change under the isometry $t \mapsto \phi_{g(t)}$ of any observer transformation $t \mapsto g(t)$, and because the minimizer of Eq. 32 is unique, as in [30], its computation is thus objective with respect to the group action $\Phi$ of the isometry group of $M$.

\section{Results}

We have implemented a general framework for flow visualization on curved surfaces, given as triangle meshes, in $\mathrm{C}++$, and OpenGL for rendering. For the sphere, we use six very simple charts, as described in detail in App. Q. We emphasize that the only 3D computations that we use are for initially obtaining the metric of the sphere as a 2D tensor field in each of the charts, and for visualization purposes in OpenGL. All our vector fields are given via 2D components, and all computations required for solving Eq. 32 are fully intrinsic in 2D. We solve the least-squares system given in Sec. 9.2 by using the conjugate gradient solvers provided by the $\mathrm{C}++$ Eigen library, obtaining the minimizer $\mathbf{u}$ in multiple iterations. We have also tried direct methods using matrix factorization of the symmetric positive definite matrix $\mathbf{A}^{T} \mathbf{W} \mathbf{A}$. Due to the size of our grids, conjugate gradients obtain the minimum faster. 
Table 1. Data sets with grid/mesh resolution.

\begin{tabular}{|c|c|c|c|c|}
\hline Data set & \# Vert. & \# Tri. & Time & Time Steps \\
\hline SYNTHETIC VORTEX & 10,242 & 20,480 & $0 \ldots 1 \mathrm{~s}$ & 100 \\
\hline SYNTHETIC FOUR CENTERS & 10,242 & 20,480 & $0 \ldots 2 \pi \mathrm{s}$ & 100 \\
\hline JUPITER VORTEX STREET & 40,962 & 81,920 & $0 \ldots \frac{\pi}{2} \mathrm{~s}$ & 300 \\
\hline EARTH FLOW & 163,842 & 327,680 & 8 days & 32 \\
\hline -EARTH FLOW (SUBDOMAIN) & 32,400 & 64,796 & 8 days & 32 \\
\hline -EARTH FLOW (ADAPT. RES.) & 62,412 & 124,820 & 8 days & 32 \\
\hline
\end{tabular}

Data sets. Table 1 gives an overview of the data sets that we have used in this paper and the video. We ran experiments with synthetic data sets and with simulated data on the sphere. The optimizations are global. For the earth flow data set we have, however, experimented with two approaches for region of interest optimization to reduce computation times. Table 2 gives computation times for the conjugate gradient solves to optimize observer fields $\mathbf{u}$. All computations were run on two Intel Xeon E5-2600 processors with 16 cores running at $2.3 \mathrm{GHz}$.

Visualization. We have adapted the observer-relative visualization methods described by Hadwiger et al. [30] from Euclidean space to curved spaces. Since we need coordinate charts to compute the optimization, we can also use them as texture parametrization for LIC computations in texture space on the GPU. Corresponding to a given observer field $\mathbf{u}$, we compute LIC images of observed stream lines [30]. Likewise, we also compute the geometry of integral curves for observed stream lines as well as for observed path lines. To visualize the time parameter of path lines, we increase the radial distance from the sphere according to the parameter $t$, scaled by a user-specified factor. This is helpful to see the time evolution of path lines. Additionally, we draw path line shadows on the sphere, to ensure that their position in space can be correctly interpreted. Moreover, we visualize time-dependent scalar fields relative to the observer field via color coding, and in the same way show texture patterns, e.g., Earth or Jupiter textures. We can dynamically switch the observer for any flow field, and directly visualize the corresponding observed fields. Figs. 1, 6, and 9 show observer-relative scalar fields (planet textures, vorticity magnitude), as well as observed LIC. Figs. 3, 6, and 8 show observed path lines.

\subsection{Synthetic Data Sets}

Synthetic Vortex. We transform a steady input field of a simple vortex into different time-dependent fields by rotating the input around the vertical axis of a sphere at different angular velocities (Fig. 3). The latter correspond to different scalings of the Killing field $\mathbf{x}_{2}$ in Fig. 5.

From each transformed, time-dependent input field, we compute the corresponding observer field $\mathbf{u}$ via optimization. In all cases, the minimizer $\mathbf{u}$ of Eq. 32 is a steady Killing field. The observer fields then enable recovering the original steady vortex in Fig. 3 (d). Relative to its observer field, each unsteady field becomes the original steady field.

Synthetic Four Centers. Fig. 8(a) shows four vortices that rotate around each other clockwise, around a common point in the center of the sphere. We show that the observer field $\mathbf{u}$ that is computed automatically via optimization recovers a steady field for the four vortices, as depicted in Fig. 8(b). In Fig. 8(c), we then observe the input field in (a) relative to a leftward rotation around the vertical axis of the

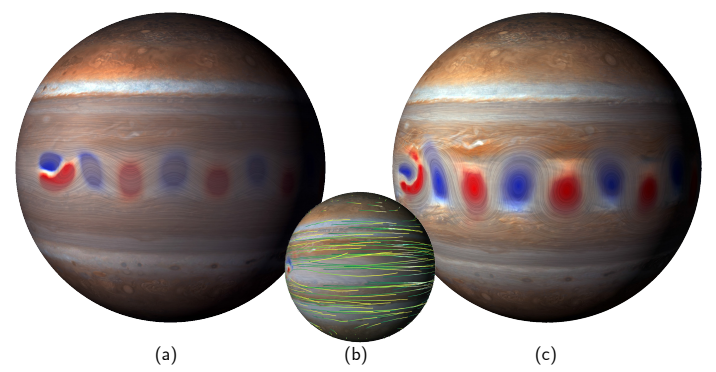

Fig. 9. Planet-scale flow patterns observed using LIC. Vorticity is color-coded on a red-blue CCW/CW scale. LIC is used to visualize instantaneous velocities. (a) A vortex street at the equator. LIC cannot show the vortices. (b) The globally optimized observer field that transforms the flow field (a) into a near-steady flow field (c), which shows the vortices in place while the planet is moving underneath instead. Note how LIC of the instantaneous velocity field now clearly shows the vortices.
Table 2. Computation times for the observer field $\mathbf{u}$.

\begin{tabular}{|c|c|c|c|c|}
\hline Data set & $\lambda$ & $\mu$ & \# CG Iter. & Comp.-time \\
\hline \hline SYNTHETIC VORTEX & 0.1 & 0 & 1,000 & $6 \mathrm{~s}$ \\
\hline SYNTHETIC FOUR CENTERS & 1 & 0 & 10,000 & $78 \mathrm{~s}$ \\
\hline JUPITER VORTEX STREET & $10^{3}$ & 0 & 5,000 & $12 \mathrm{~min}$ \\
\hline EARTH FLOW (SUBDOMAIN) & $10^{13}$ & 0 & 10,000 & $3 \mathrm{~min}$ \\
\hline EARTH FLOW (ADAPT. RES.) & $10^{13}$ & 0 & 10,000 & $6 \mathrm{~min}$ \\
\hline
\end{tabular}

sphere. Fig. 8(e) again depicts the field that is recovered automatically, this time relative to an observer field $\mathbf{u}$, shown in Fig. 8(d), that rotates clockwise as well as rightward around the vertical axis of the sphere.

\subsection{Global Planet-Scale Optimization}

Jupiter Vortex Street. Fig. 9 depicts a 2D von Kármán vortex street centered around the equator of Jupiter. We have generated this data set for testing purposes by mapping a flat vortex street to a sphere by directly mapping a Cartesian x-y grid to a latitude-longitude grid.

Earth Flow. For real planet-scale experiments we use open source flow data from the EU Copernicus project. We use global wind data to show that large phenomena like hurricane Isabel (2003) are automatically tracked by the solution of our optimization problem, although no explicit feature tracking is performed. Figs. 1 and 6 show the input flow data and the observed flow fields. We are able to compute an observed flow field that is nearly steady. To get an undistorted visualization that is centered on the hurricane, we can pick one time-dependent Killing field as the observer that travels with the hurricane. The resulting visualization applies the inverse observer motion to the Earth texture.

\subsection{Region of Interest Optimization}

Our approach optimizes for the best observer field $\mathbf{u}$ with a global optimization. However, often an optimization of the whole domain at the same resolution level is not necessary, since the user might only be interested in a smaller subdomain. We have tested two methods for this case. We either select a subdomain and run the global optimization only in this subdomain, or we adaptively remesh to obtain different, adaptive mesh resolutions for different regions on the sphere, and then run the optimization over the whole domain. The computation times can be greatly reduced from a full-resolution grid either way (Table 2).

\section{Discussion AND Conclusions}

We have presented the first objective, optimization-based framework for computing time-dependent reference frames in curved spaces with the goal of turning unsteady input flow fields into (nearly) steady fields relative to a global observer field. A major motivation for this approach is to enable feature-relative visualization and analysis of the time evolution of features, such as scalar and vector field attributes of hurricane simulations, "in place." A powerful example of this is shown in Fig. 1 (top left), where the path of hurricane Isabel is simply a path line of the automatically computed observer field $\mathbf{u}$. We have seeded this path line manually, but this could also be done algorithmically. Moreover, path lines of $\mathbf{u}$ anywhere inside the core region of the hurricane follow the general time-dependent diffeomorphism that constitutes the warping domain of the hurricane. We believe that our mathematical framework has great potential in applications such as studying features of interest in climatology and oceanography in a reference frame-independent way. Our method is objective, meaning that it is invariant with respect to any isometric observer transformation of the input flow field. This enables it to automatically follow the motion of features of interest, and makes it independent of super-imposed motions, including those of moving measurement devices capturing real-world geophysical flow phenomena. Finally, we believe that the fully intrinsic approach that we have taken, and the methodology from Riemannian geometry that we have used in our framework, could provide significant advantages and insight to other areas and applications of flow visualization as well.

\section{ACKNOWLEDGMENTS}

We thank Anna Frühstück for the illustrations and for help with the figures and the video. Hurricane Isabel data courtesy of EU Copernicus project, path from National Hurricane Center/Wikipedia. This work was supported by King Abdullah University of Science and Technology (KAUST), and the KAUST Office of Sponsored Research (OSR) award OSR-2015-CCF-2533-01. This research used resources of the Core Labs of KAUST. 


\section{REFERENCES}

[1] M. Alexa, D. Cohen-Or, and D. Levin. As-rigid-as-possible shape interpolation. In Proceedings of SIGGRAPH 2000, pp. 157-164, 2000.

[2] R. Aris. Vectors, Tensors and the Basic Equations of Fluid Mechanics. Dover Publications, Inc., 1990.

[3] V. I. Arnold. Mathematical Methods of Classical Mechanics. SpringerVerlag, 2nd ed., 1989.

[4] O. Azencot, M. Ben-Chen, F. Chazal, and M. Ovsjanikov. An operator approach to tangent vector field processing. Computer Graphics Forum, 32(5):73-82, 2013.

[5] O. Azencot, M. Ovsjanikov, F. Chazal, and M. Ben-Chen. Discrete derivatives of vector fields on surfaces-an operator approach. ACM Transactions on Graphics, 34(3):Article No. 29, 2015.

[6] S. Bachthaler, M. Strengert, D. Weiskopf, and T. Ertl. Parallel texturebased vector field visualization on curved surfaces using GPU cluster computers. In Proceedings of Eurographics Symposium on Parallel Graphics and Visualization (EGPGV), pp. 75-82, 2006.

[7] I. Baeza Rojo and T. Günther. Vector field topology of time-dependent flows in a steady reference frame. IEEE Transactions on Visualization and Computer Graphics, 26(1):280-290, 2020.

[8] H. Battke, D. Stalling, and H.-C. Hege. Fast line integral convolution for arbitrary surfaces in 3d. In Visualization and Mathematics: Experiments, Simulations, and Environments, pp. 181-195. Springer, 1997.

[9] M. Ben-Chen, A. Butscher, J. Solomon, and L. Guibas. On discrete Killing vector fields and patterns on surfaces. In Proceedings of Eurographics Symposium on Geometry Processing, pp. 1701-1711, 2010.

[10] H. Bhatia, G. Norgard, V. Pascucci, and P.-T. Bremer. The HelmholtzHodge decomposition-a survey. IEEE Transactions on Visualization and Computer Graphics, 19(8):1386-1404, 2013.

[11] H. Bhatia, V. Pascucci, and P.-T. Bremer. The natural Helmholtz-Hodge decomposition for open-boundary flow analysis. IEEE Transactions on Visualization and Computer Graphics, 20(11):1566-1578, 2014.

[12] H. Bhatia, V. Pascucci, R. M. Kirby, and P.-T. Bremer. Extracting features from time-dependent vector fields using internal reference frames. Computer Graphics Forum, 33(3):21-30, 2014

[13] R. Bujack, M. Hlawitschka, and K. I. Joy. Topology-inspired Galilean invariant vector field analysis. In Proceedings of IEEE Pacific Visualization 2016, pp. 72-79, 2016.

[14] B. Cabral and L. C. Leedom. Imaging vector fields using line integral convolution. In Proceedings of SIGGRAPH '93, pp. 263-270, 1993.

[15] G. Chen, V. Kwatra, L.-Y. Wei, C. D. Hansen, and E. Zhang. Design of $2 \mathrm{~d}$ time-varying vector fields. IEEE Transactions on Visualization and Computer Graphics, 18(10):1717-1730, 2012.

[16] G. Chen, K. Mischaikow, R. S. Laramee, P. Pilarczyk, and E. Zhang. Vector field editing and periodic orbit extraction using morse decomposition. IEEE Transactions on Visualization and Computer Graphics, 13(4):769785, 2007.

[17] F. de Goes, M. Desbrun, and Y. Tong. Vector Field Processing on Triangle Meshes. In SIGGRAPH Asia 2015 Courses, pp. 17:1-17:48, 2015.

[18] F. de Goes, B. Liu, M. Budninskiy, Y. Tong, and M. Desbrun. Discrete 2-tensor fields on triangulations. Computer Graphics Forum, 33(5):13-24, 2014.

[19] M. P. do Carmo. Riemannian Geometry. Birkhäuser, 1992.

[20] M. Edmunds, R. S. Laramee, G. Chen, N. Max, E. Zhang, and C. Ware. Surface-based flow visualization. Computers \& Graphics, 36(8):974-990, 2012.

[21] L. K. Forssell and S. D. Cohen. Using line integral convolution for flow visualization: curvilinear grids, variable-speed animation, and unsteady flows. IEEE Transactions on Visualization and Computer Graphics, 1(2):133-141, 1995.

[22] T. Frankel. The Geometry of Physics: An Introduction. Cambridge University Press, 3rd ed., 2011.

[23] A. Frishman and C. Herbert. Turbulence statistics in a two-dimensional vortex condensate. Phys. Rev. Lett., 120(20):204505, 2018.

[24] P. Grinfeld. Introduction to Tensor Analysis and the Calculus of Moving Surfaces. Springer-Verlag, 2013.

[25] T. Günther, M. Gross, and H. Theisel. Generic objective vortices for flow visualization. ACM Transactions on Graphics, 36(4):Article No. 141, 2017.

[26] T. Günther, M. Schulze, and H. Theisel. Rotation invariant vortices for flow visualization. IEEE Transactions on Visualization and Computer Graphics, 22(1):817-826, 2016.
[27] T. Günther and H. Theisel. Hyper-objective vortices. IEEE Transactions on Visualization and Computer Graphics, 25(1):1-1, 2018.

[28] T. Günther and H. Theisel. The State of the Art in Vortex Extraction. Computer Graphics Forum, 37(6):149-173, 2018.

[29] A. Hadjighasem and G. Haller. Geodesic transport barriers in Jupiter's atmosphere: A video-based analysis. SIAM Review, 58(1):69-89, 2016.

[30] M. Hadwiger, M. Mlejnek, T. Theuß1, and P. Rautek. Time-dependent flow seen through approximate observer Killing fields. IEEE Transactions on Visualization and Computer Graphics, 25(1):1257-1266, 2019.

[31] G. Haller. An objective definition of a vortex. Journal of Fluid Mechanics, 525:1-26, 2005.

[32] G. Haller, A. Hadjighasem, M. Farazmand, and F. Huhn. Defining coherent vortices objectively from the vorticity. Journal of Fluid Mechanics, 795:136-173, 2016

[33] D. D. Holm, T. Schmah, and C. Stoica. Geometric Mechanics and Symmetry: From Finite to Infinite Dimensions. Oxford University Press, 2009.

[34] G. A. Holzapfel. Nonlinear Solid Mechanics: A Continuum Approach for Engineering. Wiley, 2000.

[35] B. L. Hua and P. Klein. An exact criterion for the stirring properties of nearly two-dimensional turbulence. Physica D: Nonlinear Phenomena, 113(1):98-110, 1998.

[36] J. Huang, W. Pei, C. Wen, G. Chen, W. Chen, and H. Bao. Output-coherent image-space LIC for surface flow visualization. In Proceedings of IEEE Pacific Visualization, pp. 137-144, 2012.

[37] B. Jobard, G. Erlebacher, and M. Y. Hussaini. Lagrangian-Eulerian advection of noise and dye textures for unsteady flow visualization. IEEE Transactions on Visualization and Computer Graphics, 8(3):211-222, 2002.

[38] T. Langer, A. Belyaev, and H.-P. Seidel. Spherical barycentric coordinates. In Proceedings of Eurographics Symposium on Geometry Processing, pp. 81-88, 2006.

[39] R. S. Laramee, B. Jobard, and H. Hauser. Image space based visualization of unsteady flow on surfaces. In Proceedings of IEEE Visualization 2003, pp. 123-130, 2003.

[40] J. Laurie, G. Boffetta, G. Falkovich, I. Kolokolov, and V. Lebedev. Universal profile of the vortex condensate in two-dimensional turbulence. Phys. Rev. Lett., 113(25):254503, 2014.

[41] J. M. Lee. Introduction to Smooth Manifolds. Springer-Verlag, 2nd ed., 2012.

[42] J. M. Lee. Introduction to Riemannian Manifolds. Springer-Verlag, 2nd ed., 2018

[43] G.-S. Li, X. Tricoche, and C. Hansen. GPUFLIC: Interactive and accurate dense visualization of unsteady flows. In Proceedings of Eurovis 2006, pp. 29-34, 2006.

[44] G.-S. Li, X. Tricoche, D. Weiskopf, and C. Hansen. Flow charts: Visualization of vector fields on arbitrary surfaces. IEEE Transactions on Visualization and Computer Graphics, 14(5):1067-1080, 2008.

[45] J. E. Marsden and T. J. Hughes. Mathematical Foundations of Elasticity. Dover Publications, Inc., 1994.

[46] A. McInerney. First Steps in Differential Geometry. Springer-Verlag, 2013.

[47] S. B. Myers. Isometries of 2-dimensional riemannian manifolds into themselves. Proc. National Academy of Sciences of the USA, 22(5):297300, 1936.

[48] R. W. Ogden. Non-Linear Elastic Deformations. Dover Publications, Inc., 1997.

[49] B. O'Neill. Elementary Differential Geometry. Academic Press, revised 2nd ed., 2006

[50] J. Palacios and E. Zhang. Rotational symmetry field design on surfaces. ACM Transactions on Graphics, 26(3):Article No. 55, 2007.

[51] J. Pedlosky. Geophysical Fluid Dynamics. Springer-Verlag, 2nd ed., 1987.

[52] Z. Peng and R. S. Laramee. Higher dimensional vector field visualization: A survey. In Proceedings of EG UK Theory and Practice of Computer Graphics, pp. 149-163, 2009.

[53] P. Petersen. Riemannian Geometry. Springer-Verlag, 3rd ed., 2016.

[54] P. G. Saffman. Vortex Dynamics. Cambridge University Press, 1995.

[55] H.-W. Shen and D. L. Kao. UFLIC: A line integral convolution algorithm for visualizing unsteady flows. In Proceedings of IEEE Visualization '97, pp. 317-322, 1997

[56] J. Sokol. Flattened fluids help scientists understand oceans and atmospheres, July 2018. [Online: www.wired.com/story/ flattened-fluids-help-scientists-understand-oceans- $\backslash$ and-atmospheres; posted 27-June-2018] 
[57] J. Solomon. Discrete killing fields for pattern synthesis and symmetry detection. Undergraduate honors thesis, Stanford University, Palo Alto, CA, 2010.

[58] M. Spivak. A Comprehensive Introduction to Differential Geometry (5 volumes). Publish or Perish Press, 3rd ed., 1999.

[59] D. Stalling. LIC on surfaces. Technical report, Konrad Zuse Institute Berlin (ZIB), Berlin, Germany, 1997.

[60] D. Stalling and H.-C. Hege. Fast and resolution independent line integral convolution. In Proceedings of SIGGRAPH '95, pp. 249-256, 1995.

[61] D. Sujudi and R. Haimes. Identification of swirling flow in 3-d vector fields. In Proceedings of the 12th Computational Fluid Dynamics Conference, pp. 792-799, 1995.

[62] C. Teitzel, R. Grosso, and T. Ertl. Line integral convolution on triangulated surfaces. In Proceedings of WSCG, pp. 572-581, 1997.

[63] K. S. Thorne and R. D. Blandford. Modern Classical Physics: Optics, Fluids, Plasmas, Elasticity, Relativity, and Statistical Physics. Princeton University Press, 2017.

[64] Y. Tong, S. Lombeyda, A. N. Hirani, and M. Desbrun. Discrete multiscale vector field decomposition. In Proceedings of SIGGRAPH, pp. 445-452, 2003.

[65] C. Truesdell and W. Noll. The Nonlinear Field Theories of Mechanics. Springer-Verlag, 1965.

[66] L. W. Tu. Differential Geometry: Connections, Curvature, and Characteristic Classes. Springer-Verlag, 2017.

[67] J. J. van Wijk. Image based flow visualization. ACM Transactions on Graphics, 21(3):745-754, 2002.

[68] J. J. van Wijk. Image based flow visualization for curved surfaces. In Proceedings of IEEE Visualization 2003, pp. 123-130, 2003.

[69] A. Vaxman, M. Campen, O. Diamanti, D. Panozzo, D. Bommes, K. Hildebrandt, and M. Ben-Chen. Directional Field Synthesis, Design, and Processing. Computer Graphics Forum, 35(2):545-572, 2016.

[70] T. Weinkauf, J. Sahner, H. Theisel, and H.-C. Hege. Cores of swirling particle motion in unsteady flows. IEEE Transactions on Visualization and Computer Graphics, 13(6):1759-1766, 2007.

[71] E. Zhang, K. Mischaikow, and G. Turk. Vector field design on surfaces. ACM Transactions on Graphics, 25(4):1294-1326, 2006. 


\section{A Objective Tensor Fields of Arbitrary Order}

The definition of objective vector fields given in Eq. 10 directly generalizes to general $\left(\begin{array}{l}r \\ s\end{array}\right)$ tensor fields $\mathbf{T}\left(\omega^{1}, \ldots, \omega^{r}, \mathbf{x}_{1}, \ldots, \mathbf{x}_{s}\right),{ }^{5}$ with $r$ covector (1-form) arguments $\omega^{j}$, and $s$ vector arguments $\mathbf{x}_{i}$, because $\mathbf{T}$ is a multi-linear function of these arguments, and we can apply the definition for vectors argument-wise. We refer to App. $\mathrm{N}$ for an overview of the necessary tensor notation. We define, in abbreviated notation, that $\mathbf{T}$ is objective if, under the group action $\Phi$ with $g$, it transforms as

$$
\begin{aligned}
& \mathbf{T}^{*}\left(\left(\omega^{1}\right)^{*}, \ldots,\left(\omega^{r}\right)^{*},\left(\mathbf{x}_{1}\right)^{*}, \ldots,\left(\mathbf{x}_{S}\right)^{*}\right)= \\
& \mathbf{T}\left(g \cdot\left(\omega^{1}\right)^{*}, \ldots, g \cdot\left(\omega^{r}\right)^{*}, g^{-1} \cdot\left(\mathbf{x}_{1}\right)^{*}, \ldots, g^{-1} \cdot\left(\mathbf{x}_{S}\right)^{*}\right) .
\end{aligned}
$$

Giving the group actions precisely requires the pushforward and pullback of the diffeomorphism $\phi_{g}$, corresponding to $g \in G$, and its inverse $\phi_{g^{-1}}$. Specifically, a tensor field $\mathbf{T}$ is objective if it transforms as

$$
\begin{aligned}
& \mathbf{T}^{*}\left(\left(\omega^{1}\right)^{*}, \ldots,\left(\omega^{r}\right)^{*},\left(\mathbf{x}_{1}\right)^{*}, \ldots,\left(\mathbf{x}_{s}\right)^{*}\right)= \\
& \mathbf{T}\left(\phi_{g}^{*}\left(\omega^{1}\right)^{*}, \ldots, \phi_{g}^{*}\left(\omega^{r}\right)^{*}, \mathrm{~d} \phi_{g^{-1}}\left(\mathbf{x}_{1}\right)^{*}, \ldots, \mathrm{d} \phi_{g^{-1}}\left(\mathbf{x}_{S}\right)^{*}\right) .
\end{aligned}
$$

We refer to App. U for details on pushforwards and pullbacks. In fact, Eq. A. 2 simply states that a tensor field $\mathbf{T}$ is objective, if it transforms as $\mathbf{T}^{*}=\phi_{g^{-1}}^{*} \mathbf{T}$, meaning as the pullback of $\mathbf{T}{ }^{6}$ That is, Eq. A. 2 is identical to the definition of the pullback of a tensor $\mathbf{T}$. We also note that the pullback $\phi_{g}^{*} \omega$ of a 1 -form $\omega$ is defined via the pushforward $\mathrm{d} \phi_{g}$, i.e.,

$$
\left(\phi_{g}^{*} \omega\right)(\mathbf{v}):=\omega\left(\mathrm{d} \phi_{g}(\mathbf{v})\right)
$$

For example, a $\left(\begin{array}{l}1 \\ 1\end{array}\right)$ tensor field $\mathbf{S}$, i.e., a multi-linear map that maps one covector and one vector to a scalar, is objective if it transforms as

$$
\begin{aligned}
\mathbf{S}^{*}\left(\left(\omega^{1}\right)^{*},\left(\mathbf{x}_{1}\right)^{*}\right) & =\mathbf{S}\left(g \cdot\left(\omega^{1}\right)^{*}, g^{-1} \cdot\left(\mathbf{x}_{1}\right)^{*}\right), \\
& =\mathbf{S}\left(\phi_{g}^{*}\left(\omega^{1}\right)^{*}, \mathrm{~d} \phi_{g^{-1}}\left(\mathbf{x}_{1}\right)^{*}\right) .
\end{aligned}
$$

This is equivalent to Eq. 12, where the tensor $\mathbf{S}$ is equivalently interpreted as a linear transformation of vectors (or a vector-valued 1-form).

\section{B Additional Results}

Although in the paper we focus on spheres, as the natural underlying manifold for 2D geophysical flows, our mathematical framework applies to general curved (or flat) manifolds.

Cylinder example. As a simple example different from a sphere, Fig. 10 shows our framework applied to a cylinder, defined as a 2D manifold with boundary (the caps of the cylinder are not included in the geometry). We use two overlapping charts in this example. Fig. 10 (a) shows the input flow field as seen by an arbitrary observer. The flow field contains a vortex rotating around the cylinder. Although Line Integral Convolution shows a vortex pattern, it is not placed at the objective center of the vortex. Moreover, although the pathlines are seeded around the actual vortex, it is hard to see that they swirl around a common center. Fig. 10 (b) shows the observer field that is computed by our optimization method. For this synthetic flow field, the solution is an exact Killing field. Fig. 10 (c) shows the observed flow field. The pathlines are seeded exactly at the same positions as in Fig. 10 (a) They clearly show the swirling motion around the vortex center. The observed LIC now correctly shows the objective vortex center.

Discussion of other manifolds. In principle, our framework applies to arbitrary curved manifolds. However, in the completely general case, there are two separate major considerations: (1) Our optimization framework to compute an observer field as an approximate Killing vector field can, in fact, be used for arbitrary manifolds. (2) The concept

${ }^{5}$ For brevity, we list the arguments in this order, but the analogous applies for an argument list of contravariant and covariant arguments in any order.

${ }^{6}$ In fact, this is only possible because $\phi_{g}$ is a diffeomorphism, which guarantees that it is invertible. For non-invertible maps, the pullback of tensors of mixed variance is not defined. For diffeomorphisms, it is well-defined [41, p.321]. of objectivity with respect to isometries, however, is meaningful only for manifolds that in fact do support non-trivial continuous isometries.

(1) For any 2D manifold, our optimization framework will compute a meaningful approximate Killing field (see also the paper by Ben-Chen et al. [9]), even if the manifold does not support non-trivial exact Killing fields. The resulting observed field will be of practical use, because our minimization of the observed time derivative will make the observed field "as steady as possible," even if the input field is unsteady.

(2) A second important consideration for flow fields on general surfaces is related to the question of what an observer (or frame of reference) actually means. The most common concept of objectivity, defined with respect to time-dependent distance-preserving spatial transformations, requires reference frames between which there is a continuous family of such isometries. Then, a tensor is objective if it yields the same result for any arbitrary pair of reference frames between which such a continuous family of isometries exists. However, if the underlying manifold does not support non-trivial (non-identity map) continuous isometries, the concept of objectivity becomes extremely restricted. In fact, many general curved surfaces do not have non-trivial (non-zero) Killing fields. To be precise, our framework-including all equations referring to a general symmetry group $G$-are still correct for such manifolds, but if $G$ is set to the isometry group of that manifold it will consist of only the identity element. This can also be seen from the dimensionality of the Lie algebra of all Killing vector fields that are possible on the manifold under consideration, as explained in App. K.

We note that Marsden and Hughes [45] also describe a concept of objectivity with respect to arbitrary diffeomorphisms in elasticity. However, this then yields a much more general notion of objectivity.

\section{Guide to the Remaining Appendixes}

We give a brief overview of the appendixes, to provide a guide to their usage. We provide three different kinds of appendixes: Appendixes that (1) are related to the core of the paper, covering additional details such as derivations and proofs; (2) describe explicit computations to help with implementing our approach; and (3) give summaries of important concepts from differential geometry that we build upon in the paper.

Regarding (1), the following appendixes correspond to the core parts of the paper: We first rewrite the standard Euclidean observer transformations from continuum mechanics, as defined and described, for example, by Truesdell and Noll [65], Holzapfel [34], and Ogden [48], in a more general form in App. D, and then describe the accordingly generalized Observer transformations on general manifolds in App. E. It is not straightforward to see that an approach such as the one presented here is in fact objective. We therefore provide detailed Objectivity proofs for our method in App. F. Regarding (2) and (3), see App. C.1 and C.2, respectively. Finally, App. V provides a table summarizing the mathematical Notation used throughout the paper and the appendixes.

\section{C.1 Guide to Reimplementation}

In order to implement our approach for a curved manifold, such as the sphere, one needs to define coordinate charts. In each chart, our intrinsic approach requires the corresponding metric tensor field (comprising a $2 \times 2$ matrix for each mesh vertex; due to symmetry, only three unique values need to be stored), as well as the corresponding Christoffel symbols (comprising a $2 \times 2 \times 2$ set of values for each mesh vertex; due to symmetry, only six unique values need to be stored). Using a very simple setup of six coordinate charts orthogonally projected onto the (hemi)sphere, we give the explicit computation of the Metric and Christoffel symbols of the 2-sphere in App. Q. We note that all of these quantities are computed analytically, and therefore do not contain any numerical error. We also note that any other choice of charts for the sphere (e.g., with less distortions than orthogonal projection) can easily be used instead, and can be derived in the same way as done in App. Q. We have used orthogonally projected charts solely for simplicity.

In addition to the above analytically computed intrinsic properties of the manifold under consideration, we also require numerically approximated partial derivatives of vector components that are given numerically at mesh vertices. (Of the vector fields that we denote by $\mathbf{v}$ and $\mathbf{u}$, respectively.) We use an icosahedral mesh that triangulates 

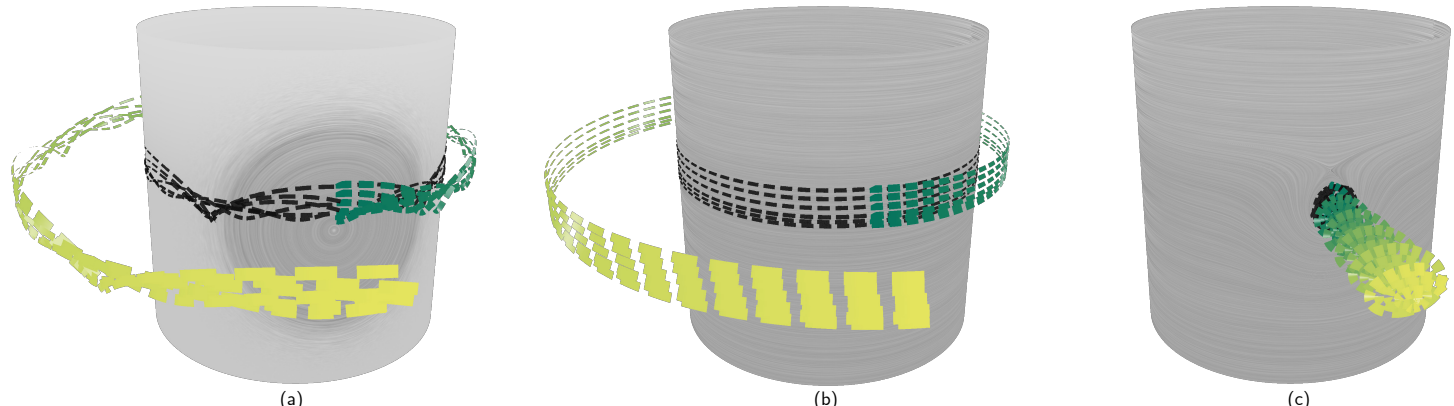

(b)

(c)

Fig. 10. Cylinder. Our framework also applies to other curved manifolds, such as this cylinder with boundary. (a) A vortex moves around the cylinder. The pathlines are seeded at the vortex core, but it is hard to see that they swirl around a common center. LIC also does not show the objective vortex center. (b) Observer field computed by optimization. (c) Observed flow field. The pathlines are seeded exactly at the same positions as in (a). They clearly show the swirling motion around the vortex center. Now LIC shows the objectively observed vortex center. Please zoom in for higher detail.

the sphere. Vector field components are given at the mesh vertices; via two components for a vector. App. R describes the corresponding Numerical computation of partial derivatives, using a simple, precomputed least-squares approach. This results in filter stencil weights for the 1-ring neighborhood of each vertex, i.e., weights for all vertices connected to the center vertex via an edge, and a weight for the center.

App. $\mathrm{H}$ describes how we compute Lie derivatives in curved spaces, which is necessary in order to compute observed time derivatives. As derived in that appendix, they can be computed solely from partial derivatives, which we estimate numerically as described in App. R.

\section{C.2 Guide to Basic Material}

We provide summaries, written using the notation that we use in the paper, of basic differential geometry concepts. These concepts are standard in differential geometry and mathematical physics, but not all of them are well-known or often used in visualization. We describe Tensors as multi-linear maps, and their bases in App. N, and describe Metric tensor fields in App. P. App. G describes Lie derivatives, and App. O describes Intrinsic covariant derivatives. App. T describes the mathematical concept of The flow of a vector field, and App. U describes the basic concepts of Pushforwards and pullbacks of a given map (often a diffeomorphism) $\phi$ between manifolds.

Symmetry groups and Lie group actions are described in App. I, the corresponding Lie algebra actions and induced vector fields in App. J, and Lie algebras and the exponential map in App. M. More specifically, we describe The Lie algebra of all Killing vector fields in App. K, and the Isometry Group and Killing Fields of the 2-Sphere in App. L.

\section{Euclidean Observer Transformations}

We repeat the spatial part of the reference frame transformation of Truesdell and Noll [65, p.41], from our Eq. 4, which is

$$
\mathbf{x}^{*}=\mathbf{c}(t)+\mathbf{Q}(t) \mathbf{x}
$$

However, this transformation is specific to Euclidean space and hard to generalize directly. The reason for this is that a general notion of time-dependent diffeomorphisms corresponding to the transformation between the frames denoted by $\mathbf{x}^{*}$ and $\mathbf{x}$, respectively, is missing. In order to prepare for-and be able to compare to-transformations on arbitrary manifolds, we introduce a one-parameter (i.e., time-dependent) family of diffeomorphisms $\phi_{t}$, mapping between the two frames, from a manifold $M=\mathbb{R}^{n}$ for the first frame (points labeled $\mathbf{x}$ ), to a manifold $N=\mathbb{R}^{n}$ for the second frame (points labeled $\mathbf{x}^{*}$ ). That is, we define

$$
\begin{aligned}
\phi_{t}: M & \rightarrow N, \\
\mathbf{x} & \mapsto \phi_{t}(\mathbf{x})=: \mathbf{x}^{*} .
\end{aligned}
$$

We equivalently also write $\phi(\mathbf{x}, t):=\phi_{t}(\mathbf{x})$. This gives simply

$$
\mathbf{x}^{*}=\phi(\mathbf{x}, t)
$$

Below, we will obtain the special case of Eq. D.1 by simply defining $\phi_{t}$ as the one-parameter family of Euclidean isometries (with $M=N=\mathbb{R}^{n}$ )

$$
\phi_{t}(\mathbf{x}):=\mathbf{c}(t)+\mathbf{Q}(t) \mathbf{x} .
$$

The map $\phi_{t}$ applies at any point $\mathbf{x} \in M$, so in order to transform a curve $t \mapsto \mathbf{x}(t) \in M$ into the corresponding curve $t \mapsto \mathbf{x}^{*}(t) \in N$, we have

$$
\mathbf{x}^{*}(t)=\phi(\mathbf{x}(t), t)
$$

Taking the derivative with respect to time $t$ on both sides, we derive that a velocity field $\mathbf{v}:=\mathrm{d} \mathbf{x}(t) / \mathrm{d} t$ transforms to $\mathbf{v}^{*}:=\mathrm{d} \mathbf{x}^{*}(t) / \mathrm{d} t$ by

$$
\begin{aligned}
\mathbf{x}^{*}(t) & =\phi(\mathbf{x}(t), t), \\
\frac{\mathrm{d}}{\mathrm{d} t} t \mapsto \mathbf{x}^{*}(t) & =\frac{\mathrm{d}}{\mathrm{d} t} t \mapsto \phi(\mathbf{x}(t), t) \\
\left(\frac{\mathrm{d}}{\mathrm{d} t} \mathbf{x}^{*}(t)\right)_{\mathbf{x}^{*}(t)} & =\left.\frac{\partial}{\partial \mathbf{x}}\right|_{\mathbf{x}=\mathbf{x}(t)} \phi(\mathbf{x}, t) \cdot\left(\frac{\mathrm{d}}{\mathrm{d} t} \mathbf{x}(t)\right)+\left.\frac{\mathrm{d}}{\mathrm{d} \tau}\right|_{\tau=t} \phi(\mathbf{x}(t), \tau), \\
\left(\frac{\mathrm{d} \mathbf{x}^{*}(t)}{\mathrm{d} t}\right)_{\mathbf{x}^{*}(t)} & =\left(\mathrm{d} \phi_{t}\right)_{\mathbf{x}(t)}\left(\frac{\mathrm{d} \mathbf{x}(t)}{\mathrm{d} t}\right)+\left.\frac{\mathrm{d}}{\mathrm{d} \tau}\right|_{\tau=t} \phi(\mathbf{x}(t), \tau) .
\end{aligned}
$$

The map d $\phi_{t}$ is the pushforward of $\phi_{t}$, i.e., $\left(\mathrm{d} \phi_{t}\right)_{\mathbf{x}}: T_{\mathbf{X}} M \rightarrow T_{\mathbf{x}^{*}} N$, with $\mathbf{x}^{*}=\phi_{t}(\mathbf{x})$. The transformation of $\mathbf{v}$ to $\mathbf{v}^{*}$, for any fixed $t$, is therefore

$$
\mathbf{v}^{*}\left(\mathbf{x}^{*}(t), t\right)=\left(\mathrm{d} \phi_{t}\right)_{\mathbf{x}(t)}(\mathbf{v}(\mathbf{x}(t), t))+\frac{\mathrm{d}}{\mathrm{d} \tau} \phi(\mathbf{x}(t), \tau) .
$$

But because Eq. D.7 is correct for any pair of positions $\left(\mathbf{x}^{*}(t), \mathbf{x}(t)\right)$, $\mathbf{x}^{*}(t)=\phi_{t}(\mathbf{x}(t))$, we can drop the position-dependence on $t$ again and write, for any corresponding pair of positions $\left(\mathbf{x}^{*}, \mathbf{x}\right)$ with $\mathbf{x}^{*}=\phi_{t}(\mathbf{x})$,

$$
\mathbf{v}^{*}\left(\mathbf{x}^{*}, t\right)=\left(\mathrm{d} \phi_{t}\right)_{\mathbf{x}}(\mathbf{v}(\mathbf{x}, t))+\frac{\mathrm{d}}{\mathrm{d} t} \phi(\mathbf{x}, t) .
$$

We can further introduce a vector field $\mathbf{w}$ on $N$, i.e., in the frame $\mathbf{x}^{*}$, as

$$
\mathbf{w}\left(\mathbf{x}^{*}, t\right):=\frac{\mathrm{d}}{\mathrm{d} t} \phi(\mathbf{x}, t), \quad \text { with } \quad \mathbf{x}=\phi_{t}^{-1}\left(\mathbf{x}^{*}\right)
$$

This allows us to write the general transformation rule as

$$
\mathbf{v}^{*}\left(\mathbf{x}^{*}, t\right)=\left(\mathrm{d} \phi_{t}\right)_{\mathbf{x}}(\mathbf{v}(\mathbf{x}, t))+\mathbf{w}\left(\mathbf{x}^{*}, t\right) .
$$

\section{D.1 Euclidean Observer Motions as Killing Fields}

For Euclidean spaces, we can write the general pushforward $\mathrm{d} \phi_{t}$ as

$$
\begin{aligned}
\left(\mathrm{d} \phi_{t}\right)_{\mathbf{x}}: T_{\mathbf{X}} M & \rightarrow T_{\mathbf{x}^{*}} N \\
\mathbf{v} & \mapsto\left(\mathrm{d} \phi_{t}\right)_{\mathbf{x}}(\mathbf{v})=\left(\frac{\partial}{\partial \mathbf{x}} \phi(\mathbf{x}, t)\right) \cdot \mathbf{v} .
\end{aligned}
$$


The specific map $\phi_{t}$ of Eq. D.4, for each time $t$, corresponds to a general isometry from Euclidean $\mathbb{R}^{n}$ to itself. The derivatives of this $\phi_{t}$ are then

$$
\begin{aligned}
\left(\mathrm{d} \phi_{t}\right)_{\mathbf{x}}=\frac{\partial}{\partial \mathbf{x}} \phi(\mathbf{x}, t) & =\mathbf{Q}(t), \\
\left(\frac{\partial}{\partial t} \phi(\mathbf{x}, t)\right)_{\mathbf{x}^{*}} & =\dot{\mathbf{c}}(t)+\dot{\mathbf{Q}}(t) \mathbf{x}, \\
& =\dot{\mathbf{c}}(t)+\dot{\mathbf{Q}}(t) \phi_{t}^{-1}\left(\mathbf{x}^{*}\right), \\
& =\dot{\mathbf{c}}(t)+\dot{\mathbf{Q}}(t) \mathbf{Q}^{T}(t)\left(\mathbf{x}^{*}-\mathbf{c}(t)\right), \\
& =\dot{\mathbf{c}}(t)+\mathbf{\Omega}(t)\left(\mathbf{x}^{*}-\mathbf{c}(t)\right), \\
& =\dot{\mathbf{c}}(t)-\boldsymbol{\Omega}(t) \mathbf{c}(t)+\boldsymbol{\Omega}(t) \mathbf{x}^{*},
\end{aligned}
$$

where $\boldsymbol{\Omega}:=\dot{\mathbf{Q}} \mathbf{Q}^{T}$ constitutes an anti-symmetric spin tensor. For brevity, we now define $\mathbf{t}(t):=\dot{\mathbf{c}}(t)-\boldsymbol{\Omega}(t) \mathbf{c}(t)$. We can then give Eq. D.8 as

$$
\mathbf{v}^{*}\left(\mathbf{x}^{*}, t\right)=\mathbf{Q}(t) \mathbf{v}(\mathbf{x}, t)+\mathbf{t}(t)+\boldsymbol{\Omega}(t) \mathbf{x}^{*} .
$$

The expression $\mathbf{t}+\boldsymbol{\Omega} \mathbf{x}^{*}$ is the derivative of a translation and rotation, i.e., an infinitesimal isometry of Euclidean space. We now see that here the vector field $\mathbf{w}$ (Eq. D.9) is $\mathbf{w}\left(\mathbf{x}^{*}, t\right)=\mathbf{t}(t)+\boldsymbol{\Omega}(t) \mathbf{x}^{*}$, and finally get

$$
\mathbf{v}^{*}\left(\mathbf{x}^{*}, t\right)=\mathbf{Q}(t) \mathbf{v}(\mathbf{x}, t)+\mathbf{w}\left(\mathbf{x}^{*}, t\right) .
$$

Or, more briefly and analogously to Eq. D.1, but probably less clearly,

$$
\mathbf{v}^{*}=\mathbf{Q}(t) \mathbf{v}+\mathbf{w}(t) .
$$

Here, $\mathbf{w}\left(\mathbf{x}^{*}, t\right)$ is a Killing vector field, because the velocity gradient tensor $\nabla \mathbf{w}$ is $\nabla \mathbf{w} \equiv \boldsymbol{\Omega}$, and thus it is anti-symmetric at all $\mathbf{x}^{*} \in N$.

In contrast to Eq. D.1, the notion of Killing fields is well-defined for arbitrary (Riemannian) manifolds, not just for $\mathbb{R}^{n}$. This inspires building on Killing vector fields, determining infinitesimal isometries, for a generalization of objectivity to isometric observer transformations.

\section{D.2 Correspondence to Our General Approach}

Our general approach is built on the group action $\Phi$ of a Lie group $G$ on a manifold $M$ (see App. I for more details), given by

$$
\begin{aligned}
\Phi: G \times M & \rightarrow M, \\
(g, x) & \mapsto \Phi(g, x) .
\end{aligned}
$$

For brevity, we denote the specific diffeomorphism corresponding to the action $\Phi$ with an element $g \in G$ by the map $\phi_{g}(x):=\Phi(g, x)$.

We can now consider the symmetry groups of Euclidean space, comprising all isometries of $\mathbb{R}^{n}$, which are often denoted as $\operatorname{ISO}(n)$ or $\mathrm{E}(n)$. However, these groups also include reflections. For our purposes of observer transformations, we want to restrict the allowed isometries to translations and rotations only. We therefore choose the subgroup $G=\mathrm{SE}(n)$, the special Euclidean group not including reflections. Each $\phi_{g}$ generated by a group element $g \in \operatorname{SE}(n)$ is then given by

$$
\begin{aligned}
\phi_{g}: \mathbb{R}^{n} & \rightarrow \mathbb{R}^{n}, \\
\mathbf{x} & \mapsto \phi_{g}(\mathbf{x})=\mathbf{c}+\mathbf{Q} \mathbf{x},
\end{aligned}
$$

where $\mathbf{c}$ is a translation vector, and $\mathbf{Q}$ is a proper orthogonal tensor.

In our framework, an observer transformation is a smooth path $t \mapsto g(t) \in G$, i.e., a one-parameter group of transformations. For $G=\operatorname{SE}(n)$, this gives each individual transformation, for a fixed $t$, as

$$
\begin{aligned}
\phi_{g(t)}: \mathbb{R}^{n} & \rightarrow \mathbb{R}^{n}, \\
\mathbf{x} & \mapsto \phi_{g(t)}(\mathbf{x})=\mathbf{c}(t)+\mathbf{Q}(t) \mathbf{x} .
\end{aligned}
$$

Given any Lie group $G$, we can consider its Lie algebra $\mathfrak{g}$, comprising the derivatives of all paths $t \mapsto h(t) \in G$ through the identity element $e$ of $G$, at the identity element $e$, i.e., the tangent space $T_{e} G$. As described in detail in App. $\mathrm{J}$, the vector field $\mathbf{w}$ generated on $M$ by a Lie algebra element $W \in \mathfrak{g}$, from the curve $t \mapsto W(t) \in \mathfrak{g}$, at a certain $t$, is given by

$$
\mathbf{w}\left(\mathbf{x}^{*}, t\right):=\left.\frac{\mathrm{d}}{\mathrm{d} \tau}\right|_{\tau=0} \phi_{h(\tau)}\left(\mathbf{x}^{*}\right), \quad \text { with } \quad h(\tau):=e^{\tau W(t)} .
$$

To understand the relationship of $t \mapsto W(t)$ with $t \mapsto g(t)$, we note that the derivative $W(t) \in T_{e} G$ maps to $g^{\prime}(t) \in T_{g(t)} G$ via left-translation $\left(L_{g}=g\right.$.) in G [22, p.399], i.e., $W(t)=\mathrm{d} L_{g^{-1}(t)}\left(g^{\prime}(t)\right)$. With this $W(t)$,

$$
\begin{aligned}
\mathbf{w}\left(\mathbf{x}^{*}, t\right) & =\left.\frac{\mathrm{d}}{\mathrm{d} \tau}\right|_{\tau=0} \phi_{h(\tau)}\left(\mathbf{x}^{*}\right)=\left.\frac{\mathrm{d}}{\mathrm{d} \tau}\right|_{\tau=0} \phi_{h(\tau)}\left(\phi_{g(t)}(\mathbf{x})\right), \\
& =\left.\frac{\mathrm{d}}{\mathrm{d} \tau}\right|_{\tau=0} \phi_{h(\tau) g(t)}(\mathbf{x})=\left.\frac{\mathrm{d}}{\mathrm{d} \tau}\right|_{\tau=t} \phi_{g(\tau)}(\mathbf{x}) .
\end{aligned}
$$

When $G$ is the isometry group of $M, \mathfrak{g}$ gives all infinitesimal isometries of $M$, and every vector field $\mathbf{w}$ is a Killing vector field on $M$, for each $t$, as already derived above specifically for the Euclidean case $M=\mathbb{R}^{n}$, i.e., compare Eq. D.20 with the second part of Eq. D.12 and below.

App. J describes Lie algebra actions and their induced vector fields in detail, and App. L describes our approach, described here for Euclidean space, instead applied to the isometries of the sphere $\mathbb{S}^{2}$.

\section{E Observer Transformations on General Manifolds}

Similar to Eq. D.18 above, we define for general manifolds $M$ and $N$,

$$
\begin{aligned}
\phi_{g(t)}: M & \rightarrow N, \\
x & \mapsto \phi_{g(t)}(x)=: x^{*} .
\end{aligned}
$$

For brevity, in the following we assume that the path $t \mapsto g(t) \in G$ is known, defining the time-dependent diffeomorphism $\phi_{t}:=\phi_{g(t)}$, i.e.,

$$
\begin{aligned}
\phi_{t}: M & \rightarrow N, \\
x & \mapsto \phi_{t}(x)=: x^{*} .
\end{aligned}
$$

To consider a curve $t \mapsto x(t) \in M$, transforming into the corresponding curve $t \mapsto x^{*}(t) \in N$, we define $\phi(x, t):=\phi_{t}(x)$ and write

$$
x^{*}(t)=\phi(x(t), t) .
$$

Now, taking the derivative with respect to the time $t$ on both sides, gives that a velocity field $\mathbf{v}:=\mathrm{d} x(t) / \mathrm{d} t$ transforms to $\mathbf{v}^{*}:=\mathrm{d} x^{*}(t) / \mathrm{d} t$ by

$$
\begin{aligned}
& x^{*}(t)=\phi(x(t), t), \\
& \frac{\mathrm{d}}{\mathrm{d} t} \mapsto x^{*}(t)=\frac{\mathrm{d}}{\mathrm{d} t} t \mapsto \phi(x(t), t), \\
&\left(\frac{\mathrm{d} x^{*}(t)}{\mathrm{d} t}\right)_{x^{*}(t)}=\left(\mathrm{d} \phi_{t}\right)_{x(t)}\left(\frac{\mathrm{d} x(t)}{\mathrm{d} t}\right)+\left.\frac{\mathrm{d}}{\mathrm{d} \tau}\right|_{\tau=t} \phi(x(t), \tau), \\
&\left(\frac{\mathrm{d} x^{*}(t)}{\mathrm{d} t}\right)_{x^{*}(t)}=\left(\mathrm{d} \phi_{t}\right)_{x(t)}\left(\frac{\mathrm{d} x(t)}{\mathrm{d} t}\right)+\mathbf{w}\left(x^{*}(t), t\right) .
\end{aligned}
$$

Here, we have introduced the vector field $\mathbf{w}$, on the manifold $N$, as

$$
\mathbf{w}\left(x^{*}, t\right):=\frac{\mathrm{d}}{\mathrm{d} t} \phi(x, t), \quad \text { with } \quad x=\phi_{t}^{-1}\left(x^{*}\right) .
$$

This gives us the general transformation rule

$$
\left(\mathbf{v}^{*}(t)\right)_{\phi_{t}(x)}=\left(\mathrm{d} \phi_{t}\right)_{x}(\mathbf{v}(t))+(\mathbf{w}(t))_{\phi_{t}(x)} .
$$

\section{F Objectivity Proofs}

According to Eq. E.6, a velocity field $\mathbf{v}$ transforms under the group action $\Phi$ with $g(t) \in G$, and corresponding diffeomorphism $\phi_{g(t)}$, as

$$
\left(\mathbf{v}^{*}(t)\right)_{\phi_{g(t)}(x)}=\left(\mathrm{d} \phi_{g(t)}\right)_{x}(\mathbf{v}(t))+(\mathbf{w}(t))_{\phi_{g(t)}(x)} .
$$

If, as above, we again define $x^{*}:=\phi_{g(t)}(x)$, we can also write this as

$$
\left(\mathbf{v}^{*}(t)\right)_{x^{*}}=\left(\mathrm{d} \phi_{g(t)}\right)_{\phi_{g(t)}^{-1}\left(x^{*}\right)}(\mathbf{v}(t))+(\mathbf{w}(t))_{x^{*}} .
$$


The vector field $\mathbf{w}$ is determined by the $\phi_{g(t)}$, and is (see also Eq. J.2),

$$
(\mathbf{w}(t))_{\phi_{g(t)}(x)}=\left.\frac{\mathrm{d}}{\mathrm{d} \tau}\right|_{\tau=t} \tau \mapsto \phi_{g(\tau)}(x) .
$$

The vector field $\mathbf{w}$ is a Killing field if and only if $\phi_{g(t)}$ is an isometry.

Euclidean space. As above, when $\phi_{g(t)}$ is an isometry of Euclidean space, the pushforward $\mathrm{d} \phi_{g(t)}$ is the same proper orthogonal (rotation) tensor $\mathbf{Q}$, i.e., $\left(\mathrm{d} \phi_{g(t)}\right)_{x}=\mathbf{Q}(t)$, at all $x \in M$. This is well-known, e.g., O'Neill [49, p.107]. In non-Euclidean spaces, however, this is not true.

\section{F.1 Objectivity of Killing Operator}

We first determine the transformation behavior of the tensor field $K \mathbf{u}$ under the group action $\Phi$ with $g \in G$, with $\phi_{g}$ an isometry. We evaluate for (arbitrary) arguments related by $\mathbf{x}^{*}=\mathrm{d} \phi_{g}(\mathbf{x})$, and $\mathbf{y}^{*}=\mathrm{d} \phi_{g}(\mathbf{y})$. For a velocity field $\mathbf{u}$ transforming as $\mathbf{u}^{*}=\mathrm{d} \phi_{g}(\mathbf{u})+\mathbf{w}$ (Eq. F.1), we get

$$
\begin{aligned}
\left(K \mathbf{u}^{*}\right)_{\phi_{g}(x)}\left(\mathbf{x}^{*}, \mathbf{y}^{*}\right) & =K\left(\left(\mathrm{~d} \phi_{g}\right)_{x}(\mathbf{u})+\mathbf{w}\right)\left(\mathbf{x}^{*}, \mathbf{y}^{*}\right), \\
& =K\left(\left(\mathrm{~d} \phi_{g}\right)_{x}(\mathbf{u})\right)\left(\mathbf{x}^{*}, \mathbf{y}^{*}\right)+K \mathbf{w}\left(\mathbf{x}^{*}, \mathbf{y}^{*}\right), \\
& =K\left(\left(\mathrm{~d} \phi_{g}\right)_{x}(\mathbf{u})\right)\left(\mathbf{x}^{*}, \mathbf{y}^{*}\right), \\
& =K\left(\left(\mathrm{~d} \phi_{g}\right)_{x}(\mathbf{u})\right)\left(\left(\mathrm{d} \phi_{g}\right)_{x}(\mathbf{x}),\left(\mathrm{d} \phi_{g}\right)_{x}(\mathbf{y})\right), \\
& =(K \mathbf{u})_{x}(\mathbf{x}, \mathbf{y}) .
\end{aligned}
$$

In the first step, we have used the linearity of the operator $K$, and in the second step $K \mathbf{w}=0$, because the Killing operator vanishes for a Killing field w. Covariant derivatives (with the Levi-Civita connection) are invariant under isometries [42, p.125], thus, from Eq. 21, the Killing operator is also invariant, giving the last step above. According to Eq. A.2, a tensor field $\mathbf{T}$ of type $\left(\begin{array}{l}0 \\ 2\end{array}\right)$ is objective, if it transforms as

$$
\mathbf{T}^{*}\left(\mathbf{x}^{*}, \mathbf{y}^{*}\right)=\mathbf{T}\left(\mathrm{d} \phi_{g^{-1}}\left(\mathbf{x}^{*}\right), \mathrm{d} \phi_{g^{-1}}\left(\mathbf{y}^{*}\right)\right) .
$$

From the transformation behavior given by Eq. F.4, we confirm that

$$
\begin{aligned}
K \mathbf{u}^{*}\left(\mathbf{x}^{*}, \mathbf{y}^{*}\right) & =K \mathbf{u}(\mathbf{x}, \mathbf{y}), \\
& =K \mathbf{u}\left(\mathrm{d} \phi_{g^{-1}}\left(\mathrm{~d} \phi_{g}(\mathbf{x})\right), \mathrm{d} \phi_{g^{-1}}\left(\mathrm{~d} \phi_{g}(\mathbf{y})\right)\right), \\
& =K \mathbf{u}\left(\mathrm{d} \phi_{g^{-1}}\left(\mathbf{x}^{*}\right), \mathrm{d} \phi_{g^{-1}}\left(\mathbf{y}^{*}\right)\right)
\end{aligned}
$$

Thus, the tensor $K \mathbf{u}$ (u arbitrary) transforms under the group action $\Phi$ with $g \in G$ according to Eq. F.5, and is therefore objective.

\section{F.2 Objectivity of Relative Velocity Fields}

We first determine the transformation behavior of the (arbitrary) relative velocity vector field $\mathbf{v}-\mathbf{u}$ under the group action $\Phi$ with $g \in G$. Since both velocity vector fields $\mathbf{v}, \mathbf{u}$ transform as $\mathbf{v}^{*}=\mathrm{d} \phi_{g}(\mathbf{v})+\mathbf{w}$, and $\mathbf{u}^{*}=\mathrm{d} \phi_{g}(\mathbf{u})+\mathbf{w}$ (Eq. F.1), respectively, we get the transformation

$$
\begin{aligned}
\left(\mathbf{v}^{*}-\mathbf{u}^{*}\right)_{\phi_{g}(x)} & =\left(\mathbf{v}^{*}\right)_{\phi_{g}(x)}-\left(\mathbf{u}^{*}\right)_{\phi_{g}(x)}, \\
& =\left(\mathrm{d} \phi_{g}\right)_{x}(\mathbf{v})+\mathbf{w}-\left(\mathrm{d} \phi_{g}\right)_{x}(\mathbf{u})-\mathbf{w}, \\
& =\left(\mathrm{d} \phi_{g}\right)_{x}(\mathbf{v})-\left(\mathrm{d} \phi_{g}\right)_{x}(\mathbf{u}), \\
& =\left(\mathrm{d} \phi_{g}\right)_{x}(\mathbf{v}-\mathbf{u}) .
\end{aligned}
$$

Thus, the relative velocity $\mathbf{v}-\mathbf{u}(\mathbf{v}, \mathbf{u}$ arbitrary) transforms under the group action $\Phi$ with $g \in G$ according to Eq. 10. Thus, it is objective.

\section{F.3 Objectivity of Lie Derivatives of Objective Tensors}

The time-dependent Lie derivative $L_{\mathbf{u}} \mathbf{v}$ of an objective vector field $\mathbf{v}$ is objective, even when the vector field $\mathbf{u}$ with respect to whose flow the Lie derivative is taken is not objective. We prove below that under the group action $\Phi$ with $g \in G, L_{\mathbf{u}} \mathbf{v}$ transforms according to Eq. 10. We start from Eq. F.1 and Eq. 10. We then follow the general proof of Marsden and Hughes [45, p.101], applied to our specific diffeomorphism $\phi_{g(t)}$.

For the proof it is crucial to keep track of which variables (spatial positions, times) are held fixed, and which are variable arguments for derivatives to be taken. We consider a fixed point $\bar{x}$ at fixed time $\bar{t}$, such that $\bar{x}=\phi_{g}(x)$ with the diffeomorphism $\phi_{g}:=\phi_{g(\bar{t})}$. That is, the corresponding fixed point $x=\phi_{g^{-1}}(\bar{x})$. Because we have to keep the point $\bar{x}$ fixed for different times $t$, we introduce the "moving source" $t \mapsto x(t):=\phi_{g^{-1}(t)}(\bar{x})$. For the time-dependence of $\mathbf{v}$, we write $\mathbf{v}_{t}:=$ $\mathbf{v}(t)$. The proof of objectivity can then be given as (with Eq. F.9 below),

$$
\begin{aligned}
\left(L_{\left.\mathbf{u}^{*} \mathbf{v}^{*}\right)_{\bar{x}}}\right. & =L_{\left(\mathrm{d} \phi_{g}\right)_{x}(\mathbf{u})+\mathbf{w}}\left(\left(\mathrm{d} \phi_{g}\right)_{x}(\mathbf{v})\right), \\
& =\mathscr{L}_{\left(\mathrm{d} \phi_{g}\right)_{x}(\mathbf{u})_{+\mathbf{w}}}\left(\left(\mathrm{d} \phi_{g}\right)_{x}(\mathbf{v})\right)+\frac{\partial}{\partial t}\left(\left(\mathrm{~d} \phi_{g(t)}\right)_{x(t)}\left(\mathbf{v}_{t}\right)\right), \\
& =\left(\mathrm{d} \phi_{g}\right)_{x}\left(\mathscr{L}_{\mathbf{u}} \mathbf{v}\right)+\mathscr{L}_{\mathbf{w}}\left(\left(\mathrm{d} \phi_{g}\right)_{x}(\mathbf{v})\right)+\frac{\partial}{\partial t}\left(\left(\mathrm{~d} \phi_{g(t)}\right)_{x(t)}\left(\mathbf{v}_{t}\right)\right), \\
& =\left(\mathrm{d} \phi_{g}\right)_{x}\left(\mathscr{L}_{\mathbf{u}} \mathbf{v}\right)+L_{\mathbf{w}}\left(\left(\mathrm{d} \phi_{g}\right)_{x}(\mathbf{v})\right), \\
& =\left(\mathrm{d} \phi_{g}\right)_{x}\left(\mathscr{L}_{\mathbf{u}} \mathbf{v}\right)+\left(\mathrm{d} \phi_{g}\right)_{x}\left(\frac{\partial \mathbf{v}}{\partial t}\right), \\
& =\left(\mathrm{d} \phi_{g}\right)_{x}\left(\mathscr{L}_{\mathbf{u}} \mathbf{v}+\frac{\partial \mathbf{v}}{\partial t}\right), \\
& =\left(\mathrm{d} \phi_{g}\right)_{x}\left(L_{\mathbf{u}} \mathbf{v}\right) .
\end{aligned}
$$

We have used that $\mathscr{L}_{\left(\mathrm{d} \phi_{g}\right)_{x}(\mathbf{u})}\left(\left(\mathrm{d} \phi_{g}\right)_{x}(\mathbf{v})\right)=\left(\mathrm{d} \phi_{g}\right)_{x}\left(\mathscr{L}_{\mathbf{u}} \mathbf{v}\right)[45, \mathrm{p} .98]$, and resolved the Lie derivative $L_{\mathbf{w}}$. For the latter, in the derivation below we insert the definition of the time-dependent Lie derivative (Eq. G.3) for $L_{\mathbf{w}}$ with flow $\psi_{t, \bar{t}}$, with pullback $\psi_{t, \bar{t}}^{*}: T_{\psi_{t, \bar{t}}(\bar{x})} M \rightarrow T_{\bar{x}} M$, define $\tilde{x}(t):=\psi_{t, \bar{t}}(\bar{x})$, and define $\hat{x}(t):=\phi_{g^{-1}(t)}(\tilde{x}(t))$. We also use that $\psi_{t, \bar{t}}=\phi_{g(t)} \circ \phi_{g^{-1}(\bar{t})}=\phi_{g(t) g^{-1}(\bar{t})}$, and rewrite, by definition, the pullback $\phi_{g(t) g^{-1}(\bar{t})}^{*}$ as the pushforward $\mathrm{d} \phi_{g(\bar{t}) g^{-1}(t)}$. This gives,

$$
\begin{aligned}
& \left(L_{\mathbf{W}}\left(\left(\mathrm{d} \phi_{g}\right)_{x}(\mathbf{v})\right)\right)_{\bar{x}}=\left.\frac{\mathrm{d}}{\mathrm{d} t}\right|_{t=\bar{t}} \psi_{t, \bar{t}}^{*}\left(\left(\mathrm{~d} \phi_{g(t)}\right)_{\hat{x}(t)}\left(\mathbf{v}_{t}\right)\right), \\
& =\left.\frac{\mathrm{d}}{\mathrm{d} t}\right|_{t=\bar{t}} \phi_{g(t) g^{-1}(\bar{t})}^{*}\left(\left(\mathrm{~d} \phi_{g(t)}\right)_{\hat{x}(t)}\left(\mathbf{v}_{t}\right)\right), \\
& =\left.\frac{\mathrm{d}}{\mathrm{d} t}\right|_{t=\bar{t}}\left(\mathrm{~d} \phi_{g(\bar{t}) g^{-1}(t)}\right)_{\tilde{x}(t)}\left(\left(\mathrm{d} \phi_{g(t)}\right)_{\hat{x}(t)}\left(\mathbf{v}_{t}\right)\right), \\
& =\left.\frac{\mathrm{d}}{\mathrm{d} t}\right|_{t=\bar{t}}\left(\mathrm{~d} \phi_{g(\bar{t})}\right)_{x}\left(\mathbf{v}_{t}\right), \\
& =\left(\mathrm{d} \phi_{g(\bar{t})}\right)_{x}\left(\left.\frac{\mathrm{d}}{\mathrm{d} t}\right|_{t=\bar{t}} \mathbf{v}_{t}\right), \\
& =\left(\mathrm{d} \phi_{g}\right)_{x}\left(\frac{\partial \mathbf{v}}{\partial t}\right) \text {. }
\end{aligned}
$$

Eqs. F.8 and F.9 prove that the Lie derivative of the objective vector field $\mathbf{v}$ is objective, even though the field $\mathbf{u}$ is not. In fact, essentially the same derivation proves that the same is true for objective tensor fields $\mathbf{t}$ of any order and variance, not only for vector fields [45, p.101].

Observed time derivative. Because in Eq. F.7 we have proved that the velocity field $\mathbf{v}-\mathbf{u}$ is objective, the proof given above immediately implies that the observed time derivative (Eq. 1) of $\mathbf{v}-\mathbf{u}$, given by

$$
\frac{\mathscr{D}}{\mathscr{D} t}(\mathbf{v}-\mathbf{u})=\frac{\partial}{\partial t}(\mathbf{v}-\mathbf{u})+\mathscr{L}_{\mathbf{u}}(\mathbf{v}-\mathbf{u})=L_{\mathbf{u}}(\mathbf{v}-\mathbf{u})
$$

is objective.

\section{G Lie Derivatives}

The Lie derivative measures the rate of change of a tensor field on a manifold $M$ with respect to the flow (App. T) generated by a vector field on $M$. For a time-independent tensor field $\mathbf{t}$, the Lie derivative $\mathscr{L}_{\mathbf{u}} \mathbf{t}$ with respect to a vector field $\mathbf{u}$ with flow $\phi_{t}$, is defined, at $x \in M$, as

$$
\left(\mathscr{L}_{\mathbf{u}} \mathbf{t}\right)_{x}:=\left.\frac{\mathrm{d}}{\mathrm{d} t}\right|_{t=0} \mathrm{~d} \phi_{-t}\left(\mathbf{t}_{\phi_{t}(x)}\right) .
$$

Here, $\mathrm{d} \phi_{t}$ is the differential of the flow $\phi_{t}$, and $\phi_{-t}=\phi_{t}^{-1}$. When $\mathbf{t}$ is a vector field $\mathbf{v}$, the Lie derivative $\mathscr{L}_{\mathbf{u}} \mathbf{v}$ is the same as the Lie bracket [22, 
Ch. 4] between the two vector fields, i.e., $\mathscr{L}_{\mathbf{u}} \mathbf{v}=[\mathbf{u}, \mathbf{v}]$. For any given torsion-free connection on a manifold $M$, such as the Levi-Civita connection, the Lie bracket, and thus the Lie derivative, is then (cf. Eq. O.7)

$$
\mathscr{L}_{\mathbf{u}} \mathbf{v}=\nabla \mathbf{v}(\mathbf{u})-\nabla \mathbf{u}(\mathbf{v}) .
$$

If the field $\mathbf{t}$ is time-dependent, the definition of the Lie derivative must be extended to the time-dependent Lie derivative [45, p.95], which is

$$
\left(L_{\mathbf{u}} \mathbf{t}\right)_{x}:=\left.\frac{\mathrm{d}}{\mathrm{d} t}\right|_{t=s} \psi_{t, s}^{*}\left(\mathbf{t}_{\psi_{t, s}(x)}\right)=\left(\frac{\partial \mathbf{t}}{\partial t}+\mathscr{L}_{\mathbf{u}} \mathbf{t}\right)_{x},
$$

at the point $x \in M$, at time $s$. The pullback $\psi_{t, s}^{*}$ is given by $\psi_{t, s}^{*}=\mathrm{d} \psi_{s, t}$. We refer to Marsden and Hughes [45, Ch. 1.6], and Frankel [22, Ch. 4].

\section{H Lie Derivatives in Curved Spaces}

Lie derivatives are independent of the metric $\mathbf{g}$ defined on the manifold $M$ [45, p.96]. For a vector field $\mathbf{v}$, this can be seen by expanding

$$
\begin{aligned}
\mathscr{L}_{\mathbf{u}} \mathbf{v} & =\nabla \mathbf{v}(\mathbf{u})-\nabla \mathbf{u}(\mathbf{v}), \\
& =\left(\nabla_{j} v^{i} u^{j}-\nabla_{j} u^{i} v^{j}\right) \mathbf{e}_{i}, \\
& =\left(\left(\partial_{j} v^{i}+\Gamma_{j k}^{i} v^{k}\right) u^{j}-\left(\partial_{j} u^{i}+\Gamma_{j k}^{i} u^{k}\right) v^{j}\right) \mathbf{e}_{i}, \\
& =\left(\partial_{j} v^{i} u^{j}+\Gamma_{j k}^{i} v^{k} u^{j}-\partial_{j} u^{i} v^{j}-\Gamma_{j k}^{i} u^{k} v^{j}\right) \mathbf{e}_{i}, \\
& =\left(\partial_{j} v^{i} u^{j}-\partial_{j} u^{i} v^{j}\right) \mathbf{e}_{i} .
\end{aligned}
$$

That is, all terms with Christoffel symbols $\Gamma_{j k}^{i}$ cancel out. This property always holds, given that the connection is torsion-free, which means that the symmetry $\Gamma_{j k}^{i}=\Gamma_{k j}^{i}$ holds (for $\left\{\mathbf{e}_{i}\right\}$ a coordinate basis). This applies in our framework, because we are using the Levi-Civita connection, which, by definition, is both metric-compatible and torsion-free.

To make parsing the tensor expressions above easier, we note that an expression like $\partial_{j} v^{i}$ can be seen as a matrix of partial derivatives, with row index $i$ and column index $j$, and $\partial_{j} v^{i} u^{j}$ is equivalent to matrixvector multiplication with a column vector $u^{j}$ with row index $j$. We also give the explicit expansion and summations for the 2D case:

$$
\begin{aligned}
\mathscr{L}_{\mathbf{u}} \mathbf{v}= & \nabla \mathbf{v}(\mathbf{u})-\nabla \mathbf{u}(\mathbf{v}), \\
= & \left(\sum_{j=1,2}\left(\left(\partial_{j} v^{1}\right) u^{j}-\left(\partial_{j} u^{1}\right) v^{j}\right)\right) \mathbf{e}_{1}+ \\
& \left(\sum_{j=1,2}\left(\left(\partial_{j} v^{2}\right) u^{j}-\left(\partial_{j} u^{2}\right) v^{j}\right)\right) \mathbf{e}_{2} .
\end{aligned}
$$

\section{Symmetry Groups ANd Lie Group Actions}

The group action $\Phi$ [33, p.209], more specifically a smooth left action, of a Lie group $G$ on a manifold $M$, is a smooth map

$$
\begin{aligned}
\Phi: G \times M & \rightarrow M, \\
(g, x) & \mapsto \Phi(g, x),
\end{aligned}
$$

such that

1. $\Phi(e, x)=x$, for all $x \in M$, and

2. $\Phi(g, \Phi(h, x))=\Phi(g h, x)$, for all $g, h \in G$ and $x \in M$,

By setting $\phi_{g}(x):=\Phi(g, x)$ the properties of the group action can be written in the more concise form

$$
\phi_{g} \phi_{h}=\phi_{g h} \quad \text { and } \quad \phi_{e}=\mathrm{id}_{M} .
$$

It follows then from $\phi_{g} \phi_{g^{-1}}=\phi_{g g^{-1}}=\phi_{e}=\operatorname{id}_{M}$ that $\phi_{g^{-1}}=\left(\phi_{g}\right)^{-1}$, and since both $\phi_{g}$ and $\phi_{g^{-1}}$ are smooth by definition that, for every $g \in G$, the map

$$
\begin{aligned}
\phi_{g}: M & \rightarrow M, \\
x & \mapsto \phi_{g}(x)
\end{aligned}
$$

is a diffeomorphism.

\section{J Lie Algebra Actions and Induced Vector Fields}

We are interested in determining a correspondence of elements of the Lie algebra $\mathfrak{g}$ of a given Lie group $G$, such as a matrix Lie group, and the space of vector fields (again as a Lie algebra) on a given manifold $M$. For example, we want to construct a correspondence between a Lie algebra of anti-symmetric matrices and the Killing vector fields on $M$.

We can do this by defining a Lie algebra homomorphism (or an isomorphism) as the action of the Lie algebra $\mathfrak{g}$ on the manifold $M$, generating vector fields on $M$. We can write this as a map

$$
\begin{aligned}
\Xi: \mathfrak{g} & \rightarrow \mathfrak{X}(M), \\
X & \mapsto \mathbf{x} .
\end{aligned}
$$

$\mathfrak{X}(M)$ denotes the Lie algebra of (smooth) vector fields on $M$. Using the Lie group action $\Phi$ with $g \in G$, and the corresponding diffeomorphism $\phi_{g}$ on $M$, the vector field $\mathbf{x}$ generated on $M$ by $X \in \mathfrak{g}$, is given by

$$
\mathbf{x}(x):=\left.\frac{\mathrm{d}}{\mathrm{d} t}\right|_{t=0} \phi_{g(t)}(x), \quad \text { with } \quad g(t):=e^{t X} .
$$

The definition of $g(t)$ on the right defines a one-parameter group of diffeomorphims $\phi_{g(t)}$ on $M$. This then means that the vector field $\mathbf{x}(x)$, at all points $x \in M$, is given by the point-wise derivative of the diffeomorphism $\phi_{g(t)}$, at each $x$, evaluated at the parameter value $t=0$.

For the common, and our, case where the Lie algebra $\mathfrak{g}$ is a matrix Lie algebra, corresponding to a matrix Lie group $G$, the exponential map $X \mapsto e^{t X}$ is given by the standard matrix exponential. See App. M.

Roughly speaking, the exponential map integrates an infinitesimal transformation $X$ (such as an infinitesimal rotation, with $X$ an antisymmetric matrix) from $g=e$, i.e., the identity element of $\mathrm{G}$, such as the identity matrix, at time $t=0$, to the corresponding finite transformation $g(t)$, at time $t$ (such as a rotation, with $g(t)$ a proper orthogonal matrix).

Appendixes $\mathrm{L}$ and $\mathrm{M}$ give a detailed description of the relationship between the isometry group $G$, the action of its Lie algebra $\mathfrak{g}$, and the corresponding vector fields on $M$, for the case of the two-sphere $\mathbb{S}^{2}$.

\section{K The Lie Algebra of all Killing Vector Fields}

The set of all possible Killing vector fields on a given (Riemannian) manifold $M$ has a lot of structure that can be exploited. In fact, the set of Killing fields constitutes a Lie algebra, which is a vector space with an additional Lie bracket operation (see App. L). Therefore, as a vector space, we can talk about the dimensionality of the space of all possible Killing fields on a manifold $M$, which we now denote by $k$.

Knowing $k$ for a given manifold $M$ gives a lot of insight on all possible isometries of $M$. For example, it is known that curved surfaces embedded in $\mathbb{R}^{3}$ can have at most three linearly independent Killing fields, i.e., they can only have $k \leq 3[9,47]$. However, a continuous isometry is a rather strong condition, and therefore many general manifolds have only the trivial intrinsic isometry $(k=0)$. In these cases, approximate Killing fields can be computed [9]. For observers and objectivity, we are interested in spaces with non-trivial isometries.

Knowing $k$ is the same as knowing the dimensionality of the isometry group of $M$. The isometry group (without reflections) of Euclidean space $\mathbb{R}^{n}$ is $\mathrm{SO}(n) \ltimes \mathrm{T}(n)$. $\mathrm{SO}(n)$ is the rotation group, $\mathrm{T}(n)$ the group of translations, and $\ltimes$ the semi-direct product. The Euclidean plane $\mathbb{R}^{2}$ thus has $k=3$; Euclidean 3-space $\mathbb{R}^{3}$ has $k=6$. The (direct) isometry group of the two-sphere $\mathbb{S}^{2}$ is $\mathrm{SO}(3)$, and therefore it has $k=3$. A cylinder has $k=2$, and a generic surface of revolution has $k=1$ [9].

\section{Isometry Group ANd Killing FieldS OF the 2-Sphere}

The (direct) isometry group of the standard two-sphere $\mathbb{S}^{2}$, embedded in the ambient space $\mathbb{R}^{3}$ as $\mathbb{S}^{2}:=\left\{(x, y, z) \in \mathbb{R}^{3}: x^{2}+y^{2}+z^{2}=1\right\}$, with the standard Euclidean topology and metric, is the Lie group $G=$ $\mathrm{SO}(3)$, which is also a smooth, non-linear manifold. $\mathrm{SO}(3)$ is a matrix Lie group, where each element $g \in G$ is a proper orthogonal matrix, with $\operatorname{det} g=1$, corresponding to a rigid rotation of $\mathbb{R}^{3}$. See also App. M. The corresponding Lie algebra $\mathfrak{g}=T_{e} G=\mathfrak{s o}(3)$ is the tangent space (i.e., a vector space) of $G$ at the identity group element $e$. As every Lie 
algebra, $\mathfrak{s o}(3)$ is a vector space with an additional Lie bracket operation, which in this case is the matrix commutator $[X, Y]:=X Y-Y X$. The Lie algebra $\mathfrak{s o}(3)$ is the algebra of all real, anti-symmetric $3 \times 3$ matrices

$$
X=\left(\begin{array}{rrr}
0 & -\omega_{3} & \omega_{2} \\
\omega_{3} & 0 & -\omega_{1} \\
-\omega_{2} & \omega_{1} & 0
\end{array}\right) \in \mathfrak{s o}(3), \text { with } \omega_{i} \in \mathbb{R}
$$

An isomorphism between a Lie algebra $\mathfrak{g}$ and vector fields induced on a manifold $M$ can be determined via a group action of $G$ on $M$, and the corresponding action of $\mathfrak{g}$, described in App. J. App. M gives an isomorphism for $\mathfrak{g}=\mathfrak{s o}(3)$ and the manifold $M=\mathbb{S}^{2}$. Using this isomorphism, each $X \in \mathfrak{s o}(3)$ corresponds to a uniquely determined Killing field $\mathbf{x}$ on $\mathbb{S}^{2}$. For the Lie algebra of vector fields $\mathbf{x}$ induced on the manifold $M$, the Lie bracket is the differential geometric Lie bracket of vector fields $[\mathbf{x}, \mathbf{y}]$, corresponding to but not the same as the matrix commutator $[X, Y]$. In fact, $[\mathbf{x}, \mathbf{y}]$ is the Lie derivative $\mathscr{L}_{\mathbf{x}} \mathbf{y}$.

The Lie algebra $\mathfrak{s o}(3)$ is three-dimensional as a vector space. This can be seen by giving a basis for all anti-symmetric $3 \times 3$ matrices, e.g., the three basis vectors $X_{1}, X_{2}, X_{3}$ in Eq. M.7. Because the Lie algebra of Killing fields on $\mathbb{S}^{2}$ and the matrix Lie algebra $\mathfrak{s o}(3)$ are isomorphic, the former is therefore also three-dimensional. Any Killing field $\mathbf{x}$ on $\mathbb{S}^{2}$ can thus be given as a unique linear combination of three basis Killing fields $\mathbf{x}_{1}, \mathbf{x}_{2}, \mathbf{x}_{3}$. Fig. 5 depicts three linearly independent basis Killing fields on $\mathbb{S}^{2}$ (we could also choose another basis), where each field $\mathbf{x}_{i}$ corresponds to a basis vector $X_{i}$ of $\mathfrak{s o}(3)$ as a vector space.

Extrinsic computation in $\mathbb{R}^{3}$. The simplest way to construct the (intrinsic) Killing field $\mathbf{x}$ on $\mathbb{S}^{2}$, corresponding to a given Lie algebra element $X \in \mathfrak{s o}(3)$, is the following. For any point $x \in M=\mathbb{S}^{2}$, given via a position vector $\mathbf{r}(x) \in \mathbb{R}^{3}$, pointing from the origin of $\mathbb{R}^{3}$ to the point $x$ on $\mathbb{S}^{2}$, the extrinsic Killing vector $\tilde{\mathbf{x}}(x) \in \mathbb{R}^{3}$ at $x$ is simply

$$
\tilde{\mathbf{x}}(x)=X \cdot \mathbf{r}(x) .
$$

(We note that this could also be written as the cross product of the angular velocity vector corresponding to $X$ with the position vector r.) From the extrinsic vector $\tilde{\mathbf{x}}(x) \in \mathbb{R}^{3}$, we can obtain the intrinsic vector $\mathbf{x} \in T_{X} M$ (two components) via the dual basis (1-forms) given in App. Q,

$$
\mathbf{x}(x)=\tilde{\omega}^{1}(\tilde{\mathbf{x}}(x)) \mathbf{e}_{1}+\tilde{\omega}^{2}(\tilde{\mathbf{x}}(x)) \mathbf{e}_{2} .
$$

\section{Lie Algebras and the Exponential Map}

The exponential map is a mapping from a Lie algebra $\mathfrak{g}$ to the corresponding Lie group $G$, i.e.,

$$
\begin{aligned}
\exp : \mathfrak{g} & \rightarrow G, \\
X & \mapsto \exp (X) .
\end{aligned}
$$

In case of a matrix Lie group it is defined for a matrix $X$ in the Lie algebra, like the exponential map for real numbers, by the power series

$$
\exp (X)=e^{X}:=\sum_{k=0}^{\infty} \frac{X^{k}}{k !}=I+X+\frac{X^{2}}{2 !}+\frac{X^{3}}{3 !}+\ldots
$$

where $I$ is the identity matrix.

The one-parameter group $g(t)$ generated by $X$ is then given by $g(t)=$ $\exp (t X)$. We immediately get the corresponding one-parameter group of actions $\Phi$ with $g(t)$, and the corresponding diffeomorphisms $\phi_{g(t)}$.

For example, for $G=\mathrm{SO}(2)$, and its Lie algebra $\mathfrak{g}=\mathfrak{s o}(2)$, if we choose the basis vector (matrix) $X \in \mathfrak{s o}(2)$

$$
X=\left(\begin{array}{rr}
0 & -1 \\
1 & 0
\end{array}\right)
$$

we can compute

$$
\begin{aligned}
& (t X)^{0}=\left(\begin{array}{ll}
1 & 0 \\
0 & 1
\end{array}\right), \\
& (t X)^{1}=\left(\begin{array}{rr}
0 & -t \\
t & 0
\end{array}\right), \\
& (t X)^{2}=\left(\begin{array}{rr}
-t^{2} & 0 \\
0 & -t^{2}
\end{array}\right), \\
& (t X)^{3}=\left(\begin{array}{rr}
0 & t^{3} \\
-t^{3} & 0
\end{array}\right), \\
& (t X)^{4}=\left(\begin{array}{rr}
t^{4} & 0 \\
0 & t^{4}
\end{array}\right)=t^{4}(t X)^{0},
\end{aligned}
$$

and conclude that $(t X)^{k+4}=t^{4}(t X)^{k}$ by induction. Thus,

$$
\begin{aligned}
\exp (t X) & =e^{t X} \\
& =\left(\begin{array}{rr}
1-\frac{t^{2}}{2 !}+\frac{t^{4}}{4 !}-\ldots & -t+\frac{t^{3}}{3 !}-\frac{t^{5}}{5 !}+\ldots \\
t-\frac{t^{3}}{3 !}+\frac{t^{5}}{5 !}-\ldots & 1-\frac{t^{2}}{2 !}+\frac{t^{4}}{4 !}-\ldots
\end{array}\right) \\
& =\left(\begin{array}{rr}
\cos t & -\sin t \\
\sin t & \cos t
\end{array}\right) \in \mathrm{SO}(2) .
\end{aligned}
$$

For $G=\mathrm{SO}(3)$, and its Lie algebra $\mathfrak{g}=\mathfrak{s o}(3)$, with essentially the same calculations, we get for the basis vectors (matrices) $X_{i} \in \mathfrak{s o}(3)$

$$
X_{1}=\left(\begin{array}{rrr}
0 & 0 & 0 \\
0 & 0 & -1 \\
0 & 1 & 0
\end{array}\right), X_{2}=\left(\begin{array}{rrr}
0 & 0 & 1 \\
0 & 0 & 0 \\
-1 & 0 & 0
\end{array}\right), X_{3}=\left(\begin{array}{rrr}
0 & -1 & 0 \\
1 & 0 & 0 \\
0 & 0 & 0
\end{array}\right)
$$

the corresponding exponentials

$$
\begin{aligned}
& \exp \left(t X_{1}\right)=\left(\begin{array}{rrr}
1 & 0 & 0 \\
0 & \cos t & -\sin t \\
0 & \sin t & \cos t
\end{array}\right) \in \operatorname{SO}(3) \\
& \exp \left(t X_{2}\right)=\left(\begin{array}{rrr}
\cos t & 0 & \sin t \\
0 & 1 & 0 \\
-\sin t & 0 & \cos t
\end{array}\right) \in \operatorname{SO}(3) \\
& \exp \left(t X_{3}\right)=\left(\begin{array}{rrr}
\cos t & -\sin t & 0 \\
\sin t & \cos t & 0 \\
0 & 0 & 1
\end{array}\right) \in \operatorname{SO}(3)
\end{aligned}
$$

\section{N Tensors as Multi-Linear Maps, and Their Bases}

We view a tensor field as a multi-linear coordinate-independent map that, at any point $x \in M$ maps a set of vector and covector (1-form) arguments to a scalar. For a detailed description, including the general concept of tensor bundles over a manifold $M$, we refer to the books by Spivak [58] and Frankel [22]. Two basic concepts that are important in our context are the order of a tensor, and its corresponding type.

We say a tensor $\mathbf{T}$ is of type $\left(\begin{array}{l}r \\ s\end{array}\right)$, and correspondingly of order $(r+s)$, if it acts on $r$ covector arguments and $s$ vector arguments.

In order to be able to work with components referred to coordinates, we have to expand tensors using basis vectors and basis covectors (basis 1-forms). Higher-order tensors must appropriately combine the basis vectors and basis 1-forms, respectively, using the tensor product $\otimes$.

We exclusively use coordinate bases (and not non-coordinate frames), where all basis vectors are derivatives of the coordinate functions $x^{i}$ of a given coordinate chart [22, p.25]. This is often denoted by $\mathbf{e}_{i}:=\boldsymbol{\partial}_{i}=\frac{\partial}{\partial x^{i}}$. This implies that the Lie brackets $\left[\mathbf{e}_{i}, \mathbf{e}_{j}\right]$ of the basis vector fields $\left\{\mathbf{e}_{i}\right\}$ are zero, i.e., the basis vector fields commute, which simplifies equations. See Frankel [22] for details. We denote the corresponding dual bases by $\left\{\omega^{i}\right\}$, with $\omega^{i}\left(\mathbf{e}_{j}\right)=\delta_{j}^{i}$. Because $\left\{\mathbf{e}_{i}\right\}$ is a coordinate basis, the $\omega^{i}$ are coordinate differentials, i.e., $\omega^{i}=d x^{i}$.

We give examples for the types of tensors that we use in this paper: 
- A vector is referred to a basis $\left\{\mathbf{e}_{i}\right\}$, and expanded as $v^{i} \mathbf{e}_{i}$.

- A 1 -form is referred to a dual basis $\left\{\omega^{i}\right\}$, and expanded as $v_{i} \omega^{i}$. We can also write $v_{i} d x^{i}$ for coordinate bases $\omega^{i}:=d x^{i}$.

- A $\left(\begin{array}{l}1 \\ 1\end{array}\right)$ tensor, as a bi-linear map of one covector and one vector argument to a scalar, is referred to a basis $\left\{\mathbf{e}_{i} \otimes \omega^{j}\right\}$, and expanded as $T_{j}^{i} \mathbf{e}_{i} \otimes \omega^{j}$. We can also interpret this as a linear map of vectors.

- A covariant second-order tensor (a $\left(\begin{array}{l}0 \\ 2\end{array}\right)$ tensor), such as the metric $\mathbf{g}$, is referred to a basis $\left\{\omega^{i} \otimes \omega^{j}\right\}$, and expanded $g_{i j} \omega^{i} \otimes \omega^{j}$.

- A contravariant second-order tensor, (a $\left(\begin{array}{l}2 \\ 0\end{array}\right)$ tensor), e.g., the inverse metric $\mathbf{g}^{-1}$, is referred to a basis $\left\{\mathbf{e}_{i} \otimes \mathbf{e}_{j}\right\}$ as $g^{i j} \mathbf{e}_{i} \otimes \mathbf{e}_{j}$.

We can understand an expression such as $T^{i}{ }_{j} \mathbf{e}_{i} \otimes \omega^{j}$ as a linear map $\mathbf{T}$, acting on a vector $\mathbf{v}$, and giving a result vector $\mathbf{T}(\mathbf{v})$, by writing

$$
\begin{aligned}
\mathbf{T}(\mathbf{v}) & =\left(T^{i}{ }_{j} \mathbf{e}_{i} \otimes \omega^{j}\right)(\mathbf{v}), \\
& =T^{i}{ }_{j} \mathbf{e}_{i} \omega^{j}(\mathbf{v})=T^{i}{ }_{j} \omega^{j}(\mathbf{v}) \mathbf{e}_{i}, \\
& =T^{i}{ }_{j} v^{j} \mathbf{e}_{i} .
\end{aligned}
$$

We note that when the 1 -form $\omega^{j}$ is applied to the vector argument $\mathbf{v}$, the tensor product $\otimes$ simply turns into a regular product. This behavior is part of the definition of the tensor product. It corresponds to the fact that the contraction $T^{i}{ }_{j} v^{j}$ turns the $\left(\begin{array}{l}1 \\ 1\end{array}\right)$ tensor $\mathbf{T}$ into a $\left(\begin{array}{l}1 \\ 0\end{array}\right)$ tensor $\mathbf{T}(\mathbf{v})$, i.e., a vector. Correspondingly, the remaining basis is solely the basis $\left\{\mathbf{e}_{i}\right\}$ for vectors. We note that the first-order tensor $\mathbf{T}(\mathbf{v})$ can be interpreted directly as a vector, or still be interpreted as a scalar-valued function acting on the argument of a covector (as one definition of a vector in tensor analysis). For example, we get the scalar that is the $i$ 'th component of the vector $\mathbf{T}(\mathbf{v})$ referred to the basis $\left\{\mathbf{e}_{i}\right\}$, by computing

$$
\mathbf{T}(\mathbf{v})\left(\omega^{i}\right):=\omega^{i}(\mathbf{T}(\mathbf{v}))=\omega^{i}\left(T^{k}{ }_{j} v^{j} \mathbf{e}_{k}\right)=T^{k}{ }_{j} v^{j} \omega^{i}\left(\mathbf{e}_{k}\right)=T^{i}{ }_{j} v^{j} .
$$

When "executed" for all "rows" $i$, the final expression $T^{i}{ }_{j} v^{j}$ is a matrixvector multiplication of components. However, in the entire derivation above, the notation has helped us avoid mixing components of different variance and the corresponding bases. Overall, tensor notation is a powerful way of using basis vectors and 1-forms, and tensors referred to components, in a general context, simplifying the use of arguments of different variance (covariant, contravariant) and higher-order tensors.

A concrete example is the definition of the covariant derivative $\nabla \mathbf{v}$ of a vector field $\mathbf{v}$ in Eqs. O.8 and O.9 below, which is a $\left(\begin{array}{l}1 \\ 1\end{array}\right)$ tensor. However, we note that in that context, "covariant" refers to "general covariance," not to covariant arguments. See Frankel [22, p.430].

\section{O Intrinsic Covariant Derivatives}

Coordinate-free definition. The covariant derivative (also called an affine connection) generalizes the directional derivative of tensor fields in Euclidean space to arbitrary manifolds [66, Chapter 6]. We define the (intrinsic) velocity gradient tensor $\nabla \mathbf{v}$ as the covariant derivative of a vector field $\mathbf{v}$ on a given manifold $M$. It has the following properties:

1. The map $(\mathbf{v}, \mathbf{w}) \mapsto \nabla_{\mathbf{w}} \mathbf{v}$ is $\mathbb{R}$-bilinear, that is

$$
\begin{aligned}
\nabla_{a \mathbf{w}_{1}+b \mathbf{w}_{2}} \mathbf{v} & =a \nabla_{\mathbf{w}_{1}} \mathbf{v}+b \nabla_{\mathbf{w}_{2}} \mathbf{v}, \quad \text { and } \\
\nabla_{\mathbf{w}}\left(a \mathbf{v}_{1}+b \mathbf{v}_{2}\right) & =a \nabla_{\mathbf{w}} \mathbf{v}_{\mathbf{1}}+b \nabla_{\mathbf{w}} \mathbf{v}_{\mathbf{2}}
\end{aligned}
$$

for all $a, b \in \mathbb{R}$.

2. The map $\mathbf{w} \mapsto \nabla_{\mathbf{w}} \mathbf{v}$ (or $\nabla \mathbf{v}(\mathbf{w})$ ) is linear with respect to smooth functions, that is

$$
\begin{aligned}
\nabla_{f \mathbf{w}_{1}+g \mathbf{w}_{2}}(\mathbf{v}) & =f \nabla_{\mathbf{w}_{1}} \mathbf{v}+g \nabla_{\mathbf{w}_{2}} \mathbf{v}, \text { or } \\
\nabla \mathbf{v}\left(f \mathbf{w}_{1}+g \mathbf{w}_{2}\right) & =f \nabla \mathbf{v}\left(\mathbf{w}_{1}\right)+g \nabla \mathbf{v}\left(\mathbf{w}_{2}\right)
\end{aligned}
$$

for all smooth functions $f, g$ on $M$.
3. The map $\mathbf{v} \mapsto \nabla_{\mathbf{w}} \mathbf{v}$ is a derivation, i.e., it satisfies the Leibniz rule

$$
\nabla_{\mathbf{w}}(f \mathbf{v})=(\mathbf{w} f) \mathbf{v}+f \nabla_{\mathbf{w}} \mathbf{v}
$$

for all smooth functions $f$ on $M$.

If we define

$$
\begin{aligned}
\nabla \mathbf{v}: \Omega^{1}(M) \times \mathfrak{X}(M) & \rightarrow C^{\infty}(M), \\
(\omega, \mathbf{w}) & \mapsto \omega(\nabla \mathbf{v}(\mathbf{w})),
\end{aligned}
$$

where $\Omega^{1}(M)$ is the space of covector fields on $M, \mathfrak{X}(M)$ the space of vector fields on $M$, and $C^{\infty}(M)$ the space of smooth functions on $M$, that is pointwise

$$
\begin{aligned}
(\nabla \mathbf{v})_{x}: T_{x}^{*} M \times T_{x} M & \rightarrow \mathbb{R} \\
\left(\omega_{x}, \mathbf{w}_{x}\right) & \mapsto \omega_{x}\left((\nabla \mathbf{v})_{x}\left(\mathbf{w}_{x}\right)\right),
\end{aligned}
$$

it follows from Eq. O.2 and the tensor characterization lemma [42, Lemma B.6] that $\nabla \mathbf{v}$ is a $\left(\begin{array}{l}1 \\ 1\end{array}\right)$ tensor field (see App. N). In addition, on a (Riemannian) manifold with a metric $\mathbf{g}$, there is a unique covariant derivative [66, Theorem 6.6] that is

1. compatible with the metric, that is ${ }^{7}$

$$
\nabla \mathbf{g}=0, \quad \text { and }
$$

2. torsion-free, that is

$$
\nabla_{\mathbf{v}} \mathbf{w}-\nabla_{\mathbf{w}} \mathbf{v}-[\mathbf{v}, \mathbf{w}]=0 .
$$

The notation $[\mathbf{v}, \mathbf{w}]$ gives the Lie bracket of the vector fields $\mathbf{v}$ and $\mathbf{w}$. This unique connection is called the Levi-Civita connection.

Computation in a chart. Referred to a coordinate basis $\left\{\mathbf{e}_{i} \otimes \omega^{j}\right\}$, the (intrinsic) velocity gradient $\nabla \mathbf{v}$ as a covariant derivative is given by

$$
\nabla \mathbf{v}=\left(\nabla_{j} v^{i}\right) \mathbf{e}_{i} \otimes \omega^{j}:=\left(\partial_{j} v^{i}+\Gamma_{j k}^{i} v^{k}\right) \mathbf{e}_{i} \otimes \omega^{j}
$$

The tensor $\nabla \mathbf{v}$ evaluated in direction $\mathbf{x}$ is the vector (see App. $\mathrm{N}$ ),

$$
\nabla \mathbf{v}(\mathbf{x})=\nabla_{\mathbf{x}} \mathbf{v}=\left(\partial_{j} v^{i}+\Gamma_{j k}^{i} v^{k}\right) \omega^{j}(\mathbf{x}) \mathbf{e}_{i}
$$

The Christoffel symbols $\Gamma_{j k}^{i}$, corresponding to the (unique) Levi-Civita connection for a metric $\mathbf{g}$ on $M$, can be derived intrinsically from the components $g_{i j}$ of $\mathbf{g}$, referred to the same basis (and its dual), via

$$
\Gamma_{j k}^{i}=\frac{1}{2} g^{i m}\left(\partial_{k} g_{m j}+\partial_{j} g_{m k}-\partial_{m} g_{j k}\right)
$$

See $\left[19\right.$, p.56]. $g_{i j}$ is the metric $\mathbf{g}$ referred to the basis $\left\{\omega^{i} \otimes \omega^{j}\right\}$, and $g^{i j}$ is its inverse $\mathbf{g}^{-1}$, i.e., $g^{i k} g_{k j}=\delta_{j}^{i}$, referred to the basis $\left\{\mathbf{e}_{i} \otimes \mathbf{e}_{j}\right\}$.

Relation to Cartesian tensors. The tensor $\nabla \mathbf{v}$ only consists solely of partial derivatives when (1) affine or Cartesian coordinates are used; and thus (2) the manifold is intrinsically flat, such as $M=\mathbb{R}^{n}$. Only then do the Christoffel symbols on $M$ vanish. The above intrinsic formulation can be used on abstract manifolds $M$, without any known immersion into a Euclidean ambient space. However, even when an immersion of $M$ into a higher-dimensional ambient space $\mathbb{R}^{m}$ is known, such as for a two-manifold embedded as a curved surface in $\mathbb{R}^{3}$, it is extremely useful for intrinsic (lower-dimensional) computations.

\footnotetext{
${ }^{7}$ This is often written in the equivalent, but less intuitive, form $\nabla_{\mathbf{u}}\langle\mathbf{v}, \mathbf{w}\rangle=$ $\left\langle\nabla_{\mathbf{u}} \mathbf{v}, \mathbf{w}\right\rangle+\left\langle\mathbf{v}, \nabla_{\mathbf{u}} \mathbf{w}\right\rangle$ [42, Proposition 5.5].
} 


\section{P Metric Tensor Fields}

Coordinate-free definition. A (Riemannian) metric $\mathbf{g}$ on a manifold $M$ defines an inner product on each tangent space $T_{x} M$. This is usually written as $\langle\mathbf{x}, \mathbf{y}\rangle:=\mathbf{g}(\mathbf{x}, \mathbf{y})$ for $\mathbf{x}, \mathbf{y} \in T_{x} M$. Specifically, $\mathbf{g}$ is

1. symmetric, that is

$$
\langle\mathbf{x}, \mathbf{y}\rangle=\langle\mathbf{y}, \mathbf{x}\rangle
$$

for all $\mathbf{x}, \mathbf{y} \in T_{x} M$,

2. bilinear, that is

$$
\langle a \mathbf{x}+b \mathbf{y}, \mathbf{z}\rangle=a\langle\mathbf{x}, \mathbf{z}\rangle+b\langle\mathbf{y} \cdot \mathbf{z}\rangle=\langle\mathbf{z}, a \mathbf{x}+b \mathbf{y}\rangle
$$

for all $\mathbf{x}, \mathbf{y}, \mathbf{z} \in T_{x} M$, and all $a, b \in \mathbb{R}$, and

3. positive definite, that is

$$
\langle\mathbf{x}, \mathbf{x}\rangle \geq 0
$$

for all $\mathbf{x} \in T_{x} M$ with $\langle\mathbf{x}, \mathbf{x}\rangle=0$ if and only if $\mathbf{x}=0$.

Furthermore, $\mathbf{g}$ is required to be smooth in the sense that in all charts the coordinate functions are smooth. Consequently, $\mathbf{g}$ is a covariant second-order tensor field (see App. N).

Computation in a chart. If we define $g_{i j}:=\left\langle\mathbf{e}_{i}, \mathbf{e}_{j}\right\rangle$, we get

$$
\begin{aligned}
\mathbf{g}(\mathbf{x}, \mathbf{y}) & =\langle\mathbf{x}, \mathbf{y}\rangle=\left\langle x^{i} \mathbf{e}_{i}, y^{j} \mathbf{e}_{j}\right\rangle=x^{i} y^{j}\left\langle\mathbf{e}_{i}, \mathbf{e}_{j}\right\rangle=x^{i} y^{j} g_{i j} \\
& =\omega^{i}(\mathbf{x}) \omega^{j}(\mathbf{y}) g_{i j}=g_{i j}\left(\omega^{i} \otimes \omega^{j}\right)(\mathbf{x}, \mathbf{y}),
\end{aligned}
$$

since $\left\{\omega^{i}\right\}$ is dual to $\left\{\mathbf{e}_{i}\right\}$, i.e., $\omega^{i}\left(\mathbf{e}_{j}\right)=\delta_{j}^{i}$, and the tensor product of two covectors is simply their product. That is, the $g_{i j}$ are the components of the metric tensor $\mathbf{g}$ with respect to the basis $\omega^{i} \otimes \omega^{j}$.

We can write $x^{i} y^{j} g_{i j}$ in matrix notation as

$$
\mathbf{x}^{T} \mathbf{g y}
$$

Inverse metric. Given a vector $\mathbf{x}=x^{i} \mathbf{e}_{i}$, the map $\mathbf{y} \mapsto \mathbf{g}(\mathbf{x}, \mathbf{y})$ defines a covector (or 1-form). From Eq. P.4, we get

$$
\begin{aligned}
\mathbf{g}(\mathbf{x}, \mathbf{y}) & =g_{i j} x^{i} \omega^{j}(\mathbf{y}), \quad \text { or } \\
(\mathbf{y} \mapsto \mathbf{g}(\mathbf{x}, \mathbf{y})) & =g_{i j} x^{i} \omega^{j} .
\end{aligned}
$$

This means that the components of the covector are simply $g_{i j} x^{i}$. If we set $x_{j}:=g_{i j} x^{i}$, we can write the covector as $x_{j} \omega^{j}$. Thus, using the metric to convert a vector into a covector, we have effectively lowered the index of the components.

The matrix $\mathbf{g}$ with components $g_{i j}$ is invertible with inverse $\mathbf{g}^{-1}$, whose components are denoted by $g^{i j}$. This means that

$$
g_{j k} g^{k i}=g^{i k} g_{k j}=\delta_{j}^{i},
$$

or in matrix notation

$$
\mathbf{g g}^{-1}=\mathbf{g}^{-1} \mathbf{g}=I,
$$

with $I$ the identity matrix. Given the components $x_{j}$ of a covector $\omega$, we now obtain the components of the corresponding vector by raising the index: $x^{i}=g^{i j} x_{j}$. See Lee [42, p. 26] for more details.

\section{Q Metric and Christoffel Symbols of the 2-Sphere}

Charts (in differential geometry). In differential geometry, a (coordinate) chart is a pair $(U, \varphi)$, where $U \subset M$ is an open subset of an $n$-dimensional manifold $M$, and the chart map $\varphi$ maps $U$ to $\mathbb{R}^{n}$, i.e., $\varphi: U \rightarrow \mathbb{R}^{n}$. To specify the chart map $\varphi$, we use $n$ coordinate functions $x^{i}: U \rightarrow \mathbb{R}$. If an atlas comprising multiple charts is used-or has to be used, as is the case for a sphere- to cover the manifold $M$, the individual charts can be labeled $\left(U_{\alpha}, \varphi_{\alpha}\right)$, where the index $\alpha$ comes from an index set. The union of all open sets $U_{\alpha}$ is required to cover all of $M$. We note that, naturally, the coordinate functions $\left\{x^{i}\right\}$ for each chart $\left(U_{\alpha}, \varphi_{\alpha}\right)$ are usually different for each chart. See, e.g., Lee [41].

Charts (simple version used below). Below, we will derive six charts covering a sphere via simple orthogonal projections for each chart. To simplify the discussion, we will denote the region of $\mathbb{R}^{2}$ that corresponds to the coordinates used in each chart by $\bar{U}$, defining $\bar{U}:=\varphi(U) \subset \mathbb{R}^{2}$. That is, $\bar{U}$ is the region in "coordinate space" for the chart $(U, \varphi)$. Furthermore, we will denote the two corresponding coordinate functions $x^{1}, x^{2}$ by $u$ and $v$ instead, i.e., we will use $u:=x^{1}$ and $v:=x^{2}$, with $u: U \rightarrow \mathbb{R}$, and $v: U \rightarrow \mathbb{R}$, respectively. In our implementation, a $(u, v)$ coordinate corresponds directly to the $2 \mathrm{D}$ texture coordinates that we use, for example, for LIC computations.

Coordinate bases, derivatives, metric, Christoffel symbols. We now explicitly derive the coordinate bases and their derivatives of charts $(U, \varphi)$ on a sphere mapping to the coordinate region $\bar{U} \subset \mathbb{R}^{2}$, corresponding to orthogonal projection of the region $\bar{U}$ onto a hemisphere of some arbitrary radius $r$. See Fig. 11. From these, we can derive the metric tensor and the Christoffel symbols referred to the chart, given analytically for any position referenced by $(u, v)$ coordinates. Furthermore, although we use six charts to cover the sphere, below we mainly derive a single chart, because all other charts are completely analogous. Even more simple, the metric as well as the Christoffel symbols that we derive for one chart are identical for all other charts, due to symmetry.

We emphasize that, although below we perform some derivations in the ambient space $\mathbb{R}^{3}$, the resulting metric (Eq. Q.6) and the Christoffel symbols (Eq. Q.9) are completely intrinsic, i.e., independent of the immersion in $\mathbb{R}^{3}$, and correspondingly are $2 \mathrm{D}$ quantities. Using the Christoffel symbols, we can compute the covariant derivative of any vector field $\mathbf{v}$ (Eq. Q.12, Eq. O.8) in a completely intrinsic manner.

Due to projection, each region $\bar{U} \subset \mathbb{R}^{2}$ is limited to an (open) disk of radius $r$ in the $(u, v)$ plane, i.e., $\bar{U}=\left\{(u, v): u^{2}+v^{2}<r^{2}\right\}$. We describe the map from $\bar{U} \subset \mathbb{R}^{2}$ (intrinsic view; corresponding to $U$ on the manifold $M$ ) to ambient $\mathbb{R}^{3}$ (extrinsic view) via the inclusion map

$$
\begin{aligned}
\imath^{x \perp y}: \bar{U} & \subset \mathbb{R}^{2} \hookrightarrow \mathbb{R}^{3}, \\
(u, v) & \mapsto(u, v, \bar{w}) .
\end{aligned}
$$

The third component is $\bar{w}:=\sqrt{r^{2}-u^{2}-v^{2}}$. This chart is defined via projection onto the hemisphere on the $x, y$ plane, denoted by $x \perp y$. The entire sphere is covered by six analogous charts. In total, we define

$$
\begin{aligned}
& \imath^{x \perp y}:(u, v) \mapsto(u, v, \bar{w}), \quad \imath^{-x \perp y}:(u, v) \mapsto(-u, v,-\bar{w}), \\
& \imath^{z \perp y}:(u, v) \mapsto(-\bar{w}, v, u), \quad \imath^{-z \perp y}:(u, v) \mapsto(\bar{w}, v,-u), \\
& \imath^{x \perp-z}:(u, v) \mapsto(u, \bar{w},-v), \quad \imath^{x \perp z}:(u, v) \mapsto(u,-\bar{w}, v) .
\end{aligned}
$$

To avoid too severe distortions, apart from overlaps to facilitate transitions between neighboring charts, each chart is only used where $u^{2} \leq \bar{w}^{2}$ and $v^{2} \leq \bar{w}^{2}$. Outside this region, another chart will be used.

We now consider the basis vectors $\frac{\partial}{\partial x^{i}}=\boldsymbol{\partial}_{i}=\mathbf{e}_{i}, i \in\{1,2\}$, denoting coordinate functions $x^{1}, x^{2}:=u, v$. In the chart, $\mathbf{e}_{1}, \mathbf{e}_{2}$ are by definition

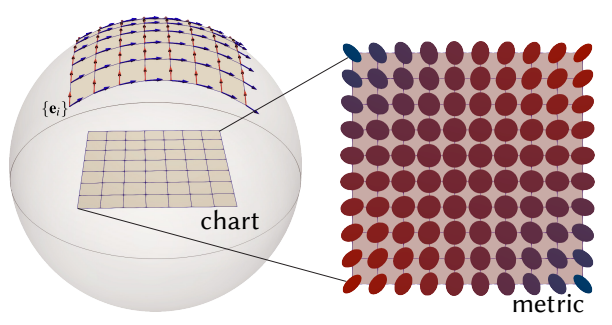

Fig. 11. Intrinsic properties of the sphere. We describe everything intrinsically in $2 \mathrm{D}$ coordinate charts. At each coordinate $(u, v)$ in a region $\bar{U} \subset \mathbb{R}^{2}$, corresponding to the open set $U$ on the sphere, with $\bar{U}=\varphi(U)$, we know the corresponding metric tensor (glyph visualization on the right) in components $g_{i j}$, and the corresponding Christoffel symbols $\Gamma_{j k}^{i}$. 
given by components $(1,0),(0,1)$, respectively. In ambient space $\mathbb{R}^{3}$, for the chart $x \perp y$, they map to the partial derivatives of Eq. Q.1, i.e.,

$$
\left.\tilde{\mathbf{e}}_{1}\right|_{(u, v)}=\left(\begin{array}{c}
1 \\
0 \\
-u / \bar{w}
\end{array}\right),\left.\quad \tilde{\mathbf{e}}_{2}\right|_{(u, v)}=\left(\begin{array}{c}
0 \\
1 \\
-v / \bar{w}
\end{array}\right) .
$$

These components are referred to Cartesian coordinates in $\mathbb{R}^{3}$. We will now also use the shorthand notations $a^{2}:=r^{2}-u^{2}, b^{2}:=r^{2}-v^{2}$. The dual basis $\omega^{i}$, with $\omega^{i}\left(\mathbf{e}_{j}\right)=\delta_{j}^{i}$, mapped to ambient space $\mathbb{R}^{3}$, is

$$
\left.\tilde{\omega}^{1}\right|_{(u, v)}=\frac{1}{r^{2}}\left(\begin{array}{c}
a^{2} \\
-u v \\
-u \bar{w}
\end{array}\right),\left.\quad \tilde{\omega}^{2}\right|_{(u, v)}=\frac{1}{r^{2}}\left(\begin{array}{c}
-u v \\
b^{2} \\
-v \bar{w}
\end{array}\right) .
$$

In order to be able to directly use Eq. Q.8 below, these two dual basis vectors $\widetilde{\omega}^{1}$ and $\tilde{\omega}^{2}$ were computed such that they correspond to orthogonal projection from the ambient space $\mathbb{R}^{3}$ into the tangent plane of the immersion of $M$ into $\mathbb{R}^{3}$. An easy way to do this is to compute an orthogonal third extrinsic basis vector $\tilde{\mathbf{e}}_{3}:=\tilde{\mathbf{e}}_{1} \times \tilde{\mathbf{e}}_{2}$, and compute the extrinsic dual basis by inverting the $3 \times 3$ matrix with columns $\left\{\tilde{\mathbf{e}}_{i}\right\}$ to get $\left\{\tilde{\omega}^{i}\right\}$. The basis vector $\tilde{\mathbf{e}}_{3}$, and its corresponding dual $\tilde{\omega}^{3}$, are

$$
\left.\tilde{\mathbf{e}}_{3}\right|_{(u, v)}=\left(\begin{array}{c}
u / \bar{w} \\
v / \bar{w} \\
1
\end{array}\right),\left.\quad \tilde{\omega}^{3}\right|_{(u, v)}=\frac{1}{r^{2}}\left(\begin{array}{c}
u \bar{w} \\
v \bar{w} \\
\bar{w}^{2}
\end{array}\right) .
$$

The components of the intrinsic metric tensor $\mathbf{g}$ can then be computed as $g_{i j}=\tilde{\mathbf{e}}_{i} \cdot \tilde{\mathbf{e}}_{j}$, with - the usual Euclidean dot product. For our chart, the metric $\mathbf{g}$ (components $g_{i j}$ ) and its inverse $\mathbf{g}^{-1}$ (components $g^{i j}$ ), are

$$
\left.g_{i j}\right|_{(u, v)}=\frac{1}{\bar{w}^{2}}\left[\begin{array}{cc}
b^{2} & u v \\
u v & a^{2}
\end{array}\right],\left.\quad g^{i j}\right|_{(u, v)}=\frac{1}{r^{2}}\left[\begin{array}{cc}
a^{2} & -u v \\
-u v & b^{2}
\end{array}\right] .
$$

The partial derivatives of the basis vectors in ambient $\mathbb{R}^{3}$, in the directions $x^{1}, x^{2}:=u, v$, evaluated at the coordinate $(u, v) \in \bar{U} \subset \mathbb{R}^{2}$, are

$$
\begin{aligned}
& \left.\partial_{1} \tilde{\mathbf{e}}_{1}\right|_{(u, v)}=-\frac{1}{\bar{w}^{3}}\left(\begin{array}{c}
0 \\
0 \\
b^{2}
\end{array}\right),\left.\quad \partial_{1} \tilde{\mathbf{e}}_{2}\right|_{(u, v)}=-\frac{1}{\bar{w}^{3}}\left(\begin{array}{c}
0 \\
0 \\
u v
\end{array}\right), \\
& \left.\partial_{2} \tilde{\mathbf{e}}_{1}\right|_{(u, v)}=-\frac{1}{\bar{w}^{3}}\left(\begin{array}{c}
0 \\
0 \\
u v
\end{array}\right),\left.\quad \partial_{2} \tilde{\mathbf{e}}_{2}\right|_{(u, v)}=-\frac{1}{\bar{w}^{3}}\left(\begin{array}{c}
0 \\
0 \\
a^{2}
\end{array}\right) .
\end{aligned}
$$

From the immersion in $\mathbb{R}^{3}$, we can now derive the Christoffel symbols $\Gamma^{i}{ }_{j k}$. From the basis vector field partial derivatives $\partial_{j} \tilde{\mathbf{e}}_{i}$ just computed, reading off components in the tangent plane with the dual basis gives

$$
\Gamma_{j k}^{i}=\tilde{\omega}^{i}\left(\partial_{j} \tilde{\mathbf{e}}_{k}\right), \quad \text { for } i, j, k \in\{1,2\} .
$$

Due to the way in which we have computed the dual basis $\left\{\tilde{\omega}^{1}, \tilde{\omega}^{2}\right\}$, this is equivalent to a completely intrinsic computation from the metric using Eq. O.10, but easier to compute. We emphasize that using this extrinsic "shortcut" computation does not in any way change the fact that afterwards we can perform all computations requiring Christoffel symbols, i.e., covariant derivatives, in a fully intrinsic manner.

The Christoffel symbols that we need, given with respect to $(u, v) \in$ $\bar{U} \subset \mathbb{R}^{2}$, are (only six are unique, because $\Gamma_{12}^{1}=\Gamma_{21}^{1}, \Gamma_{12}^{2}=\Gamma_{21}^{2}$ ),

$$
\begin{aligned}
& \left.\Gamma_{11}^{1}\right|_{(u, v)}=c u b^{2},\left.\quad \Gamma_{21}^{1}\right|_{(u, v)}=c u^{2} v, \\
& \left.\Gamma_{12}^{1}\right|_{(u, v)}=c u^{2} v,\left.\quad \Gamma_{22}^{1}\right|_{(u, v)}=c u a^{2}, \\
& \left.\Gamma_{11}^{2}\right|_{(u, v)}=c v b^{2},\left.\quad \Gamma_{21}^{2}\right|_{(u, v)}=c u v^{2}, \\
& \left.\Gamma_{12}^{2}\right|_{(u, v)}=c u v^{2},\left.\quad \Gamma_{22}^{2}\right|_{(u, v)}=c v a^{2} .
\end{aligned}
$$

Here, we have used the shorthand $c:=1 /\left(r^{2} \bar{w}^{2}\right)$. One can verify that with these Christoffel symbols we now have, extrinsically in $\mathbb{R}^{3}$,

$$
\nabla_{\tilde{\mathbf{e}}_{j}} \tilde{\mathbf{e}}_{k}=\Gamma_{j k}^{i} \tilde{\mathbf{e}}_{i}, \quad \text { for } i, j, k \in\{1,2\},
$$

where $\nabla_{\tilde{\mathbf{e}}_{j}} \tilde{\mathbf{e}}_{k}$ always lies in the tangent plane at the point corresponding to $(u, v)$. However, most importantly, we now never need to refer to the ambient space $\mathbb{R}^{3}$ again, and can compute everything intrinsically in the chart, with the same values for the Christoffel symbols $\Gamma_{j k}^{i}$, giving

$$
\nabla_{\mathbf{e}_{j}} \mathbf{e}_{k}=\Gamma_{j k}^{i} \mathbf{e}_{i}, \quad \text { for } i, j, k \in\{1,2\} .
$$

Because the covariant derivative is linear in each of its arguments, Eq. Q.11 determines Eq. O.8 for the covariant derivative $\nabla \mathbf{v}$ of any vector field v. In a $2 \mathrm{D}$ chart, we can thus expand Eq. 0.8 as the matrix

$$
\left[\begin{array}{ll}
\nabla_{1} v^{1} & \nabla_{2} v^{1} \\
\nabla_{1} v^{2} & \nabla_{2} v^{2}
\end{array}\right]=\left[\begin{array}{ll}
\partial_{1} v^{1}+\Gamma_{11}^{1} v^{1}+\Gamma_{12}^{1} v^{2} & \partial_{2} v^{1}+\Gamma_{21}^{1} v^{1}+\Gamma_{22}^{1} v^{2} \\
\partial_{1} v^{2}+\Gamma_{11}^{2} v^{1}+\Gamma_{12}^{2} v^{2} & \partial_{2} v^{2}+\Gamma_{21}^{2} v^{1}+\Gamma_{22}^{2} v^{2}
\end{array}\right] .
$$

Evaluating $\nabla_{\mathbf{x}} \mathbf{v}=\nabla \mathbf{v}(\mathbf{x})$ (Eq. O.9) in the chart thus becomes a matrixvector multiply of the matrix $\nabla_{j} v^{i}$, times the vector components $x^{i}$.

All charts. Due to the symmetry of all charts, the metric components (Eq. Q.6) and the Christoffel symbols (Eq. Q.9) are the same in all charts, although above we have derived them only for the chart $x \perp y$.

\section{R Computing Partial Derivatives}

We again work with coordinates in $\bar{U} \subset \mathbb{R}^{2}$. Each $\bar{U}$ is triangulated, with mesh vertices $\left\{x_{k}\right\}$ at $2 \mathrm{D}$ coordinates $\left(u\left(x_{k}\right), v\left(x_{k}\right)\right)=\left(u_{k}, v_{k}\right) \in \mathbb{R}^{2}$. To compute the partial derivatives $\partial_{1} v^{i}$ and $\partial_{2} v^{i}$ of an $\mathbb{R}$-valued function $v^{i}(x)$ given at the vertices, we consider the 1 -form $d v^{i}$, with basis $\left\{\omega^{i}\right\}$,

$$
d v^{i}=\left(\partial_{1} v^{i}\right) \omega^{1}+\left(\partial_{2} v^{i}\right) \omega^{2}
$$

To compute $d v^{i}$ for a single triangle comprising the vertices $x_{0}, x_{1}, x_{2}$, with coordinates $\left(u_{0}, v_{0}\right),\left(u_{1}, v_{1}\right),\left(u_{2}, v_{2}\right) \in \mathbb{R}^{2}$, and known function values $v^{i}\left(x_{0}\right), v^{i}\left(x_{1}\right), v^{i}\left(x_{2}\right) \in \mathbb{R}$, we can solve the $2 \times 2$ linear system

$$
\left[\begin{array}{ll}
\left(u_{1}-u_{0}\right) & \left(v_{1}-v_{0}\right) \\
\left(u_{2}-u_{0}\right) & \left(v_{2}-v_{0}\right)
\end{array}\right]\left[\begin{array}{l}
\partial_{1} v^{i} \\
\partial_{2} v^{i}
\end{array}\right]=\left[\begin{array}{l}
v^{i}\left(x_{1}\right)-v^{i}\left(x_{0}\right) \\
v^{i}\left(x_{2}\right)-v^{i}\left(x_{0}\right)
\end{array}\right]
$$

in order to obtain $\partial_{1} v^{i}$ and $\partial_{2} v^{i}$. For a 1-ring around a given vertex $x_{0}$ (see Fig. 12), labeling its vertices as $x_{0}, x_{1}, x_{2}, x_{3}, \ldots, x_{n-1}$, we can solve, in the least-squares sense, the over-determined $(n-1) \times 2$ system

$$
\left[\begin{array}{cc}
\left(u_{1}-u_{0}\right) & \left(v_{1}-v_{0}\right) \\
\left(u_{2}-u_{0}\right) & \left(v_{2}-v_{0}\right) \\
\vdots & \vdots \\
\left(u_{n-1}-u_{0}\right) & \left(v_{n-1}-v_{0}\right)
\end{array}\right]\left[\begin{array}{l}
\partial_{1} v^{i} \\
\partial_{2} v^{i}
\end{array}\right]=\left[\begin{array}{c}
v^{i}\left(x_{1}\right)-v^{i}\left(x_{0}\right) \\
v^{i}\left(x_{2}\right)-v^{i}\left(x_{0}\right) \\
\vdots \\
v^{i}\left(x_{n-1}\right)-v^{i}\left(x_{0}\right)
\end{array}\right] .
$$

If we write the system above in the abbreviated form $\mathbf{A d}=\mathbf{v}$, we can solve the $2 \times 2$ square system $\mathbf{A}^{T} \mathbf{A} \mathbf{d}=\mathbf{A}^{T} \mathbf{v}$, i.e., $\mathbf{d}=\left(\mathbf{A}^{T} \mathbf{A}\right)^{-1} \mathbf{A}^{T} \mathbf{v}$, corresponding to the normal equations of the least-squares problem.

We can simplify the structure of this computation by computing weights $\left\{\left(w_{j}^{1}, w_{j}^{2}\right)\right\}_{j=0}^{n-1}$ for each vertex $x_{j}$ in the 1-ring of vertex $x_{0}$. These weights form an $n$-tap filter stencil for computing a weighted average of the 1-ring neighborhood of vertex $x_{0}$. From them, we can compute the components $\partial_{1} v^{i}, \partial_{2} v^{i}$ of the 1 -form $d v^{i}$ at vertex $x_{0}$ as

$$
\begin{aligned}
& \left.\partial_{1} v^{i}\right|_{\left(u_{0}, v_{0}\right)}=w_{0}^{1} v^{i}\left(x_{0}\right)+w_{1}^{1} v^{i}\left(x_{1}\right)+\ldots+w_{n-1}^{1} v^{i}\left(x_{n-1}\right), \\
& \left.\partial_{2} v^{i}\right|_{\left(u_{0}, v_{0}\right)}=w_{0}^{2} v^{i}\left(x_{0}\right)+w_{1}^{2} v^{i}\left(x_{1}\right)+\ldots+w_{n-1}^{2} v^{i}\left(x_{n-1}\right) .
\end{aligned}
$$

In order to compute all weights $\left\{\left(w_{j}^{1}, w_{j}^{2}\right)\right\}_{j=0}^{n-1}$ in the stencil, we introduce the $2 \times(n-1)$ matrix $\mathbf{W}:=\left(\mathbf{A}^{T} \mathbf{A}\right)^{-1} \mathbf{A}^{T}$, labeling components as 


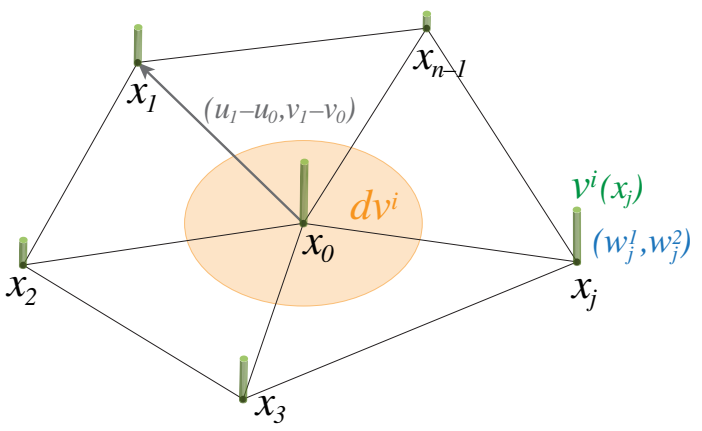

Fig. 12. 1-ring neighborhood of a triangle vertex $x_{0}$ for approximating 1 -forms $d v^{i}=\left(\partial_{1} v^{i}\right) \omega^{1}+\left(\partial_{2} v^{i}\right) \omega^{2}$ of $\mathbb{R}$-valued functions $v^{i}$ on $M$.

$\mathbf{W}_{i j}$, with $i$ the row and $j$ the column index, respectively. Considering the structure of the $(n-1) \times 1$ right-hand side above, we directly obtain

$$
\begin{array}{ll}
w_{0}^{i}=-\sum_{j=1}^{n-1} \mathbf{W}_{i j}, & i \in\{1,2\}, \\
w_{j}^{i}=\mathbf{W}_{i j}, & i \in\{1,2\} ; 1 \leq j \leq(n-1) .
\end{array}
$$

We pre-compute the $2 n$ weights of each 1-ring neighborhood, with $n$ vertices, storing them with the corresponding center vertex (above: $x_{0}$ ).

We note that all filter stencils depend solely on the geometry (the vertex positions) of the triangle mesh, but not on any specific function $v^{i}(x)$. We can therefore associate the filter weights with each triangle vertex, and then use them to compute the partial derivatives of arbitrary functions, e.g., the $v^{1}$ and $v^{2}$ of the previous section, see Eq. Q.12. We also emphasize that these partial derivatives are the only numerically approximated quantities. The metric components $g_{i j}$ (Eq. Q.6) and the Christoffel symbols $\Gamma^{i}{ }_{j k}$ (Eq. Q.9) are accurately computed analytically.

\section{S Multi-Chart Optimization}

From a general (geometric) perspective, every point $x \in M$ has exactly one corresponding vector $\mathbf{u}(x)$ from a vector field $\mathbf{u}$ given on the manifold $M$. However, for storage and computation, we store each vector $\mathbf{u}(x)$ using two components referring the vector to a basis $\left\{\mathbf{e}_{1}, \mathbf{e}_{2}\right\}$ for the tangent space $T_{x} M$. If the manifold is covered by a single chart, this is straightforward. If multiple charts are used, however, each point $x$ that maps to more than one chart also has more than one basis for the tangent space $T_{x} M$, i.e., one basis $\mathbf{e}_{i}:=\frac{\partial}{\partial x^{i}}$ corresponding to each chart with coordinate functions $x^{i}$. Since we compute the observer field $\mathbf{u}$ via optimization, computing two unknown components for each vector $\mathbf{u}(x)$, we want to optimize for each vector using only a single chart, and thus a single basis, even when multiple charts cover the position $x$. We do this by arbitrarily choosing one chart for each point $x$, for which the vector components will be computed by solving the least-squares problem given in Sec. 9.2. However, the numerical computation of partial derivatives is built on computing finite differences, as described in App. R. In regions where multiple charts overlap, there will be vertices whose 1-ring vertex neighborhood has not been assigned to the same chart. However, it is crucial that all finite differences for any given vertex are computed from vector components that all come from the same chart, because otherwise the differences are meaningless.

We handle this problem in a very simple way. For any given vertex, we can compute a $2 \times 2$ Jacobian matrix that transforms the vector components referred to a given chart to any other chart that overlaps the same vertex. This is simply a change-of-basis matrix from one chart to another. Since our entire optimization is based on solving a large linear system, the components of these Jacobian matrices can be inserted into the constituent matrices $\mathbf{K}$ and $\mathbf{D}$ (Sec. 9.1) such that they accept unknown vector components for the unknown vector $\mathbf{u}$ (Secs. 9.1 and 9.2), and transform them into the correct chart for finite difference computations in any given 1-ring vertex neighborhood. Finite differences are computed by inserting the filter stencil weights described in App. R into the corresponding matrix locations, which can be directly combined with the Jacobian change-of-basis matrix components. In this way, we "bake" all required chart transition maps into the linear system before solving it, resulting in the correct estimation of all partial derivatives at each vertex of the triangle mesh representing the manifold $M$.

\section{T THE Flow OF A Vector FIELD}

We briefly summarize the standard concepts of the flow of a vector field as they are typically defined in differential geometry. This appendix is the same as the corresponding one in Hadwiger et al. [30, App. B].

The flow of a time-independent vector field $\mathbf{u}$ on a manifold $M$ is a map $\phi: J \times M \rightarrow M$ for a suitable interval $J \subseteq \mathbb{R}$, such that $t \mapsto \phi(t, x)$ is the unique maximal integral curve of $\mathbf{u}$ through $x \in M$ [41, Th. 9.12]. That is, $\phi$ maps a point $x$ to its image along the integral curve of $\mathbf{u}$ after time $t$, which we also denote by $\phi_{t}(x)$. Important properties of $\phi$ are:

- The map $\phi_{t}: M \rightarrow M$ is a (local) diffeomorphism for all $t \in J$.

- For all $t_{1}, t_{2} \in J, x \in M, \phi_{t_{2}}\left(\phi_{t_{1}}(x)\right)=\phi_{t_{1}+t_{2}}(x), \phi_{0}(x)=x$. The inverse of $\phi_{t}$ is $\phi_{-t}$, i.e., $\phi_{t}^{-1}\left(\phi_{t}(x)\right)=\phi_{-t}\left(\phi_{t}(x)\right)=x$. $\phi$ is an action of the additive group $\mathbb{R}$ on $M, \phi_{t}$ is a one-parameter group.

- The linear map d $\phi_{t}: T_{x} M \rightarrow T_{\phi_{t}(x)} M$, called the differential of $\phi_{t}$, or the (pointwise) push-forward, is an isomorphism between the two tangent spaces at each $x \in M$ and $\phi_{t}(x) \in M$, for each $t \in J$. $\mathrm{d} \phi_{t}$ maps tangent vectors to all possible curves through a point $x \in M$ to the corresponding tangent vectors of the images of these curves under the diffeomorphism $\phi_{t}$, through the point $\phi_{t}(x) \in M$.

When the vector field $\mathbf{u}$ is time-dependent, the corresponding timedependent flow $\psi: J \times J \times M \rightarrow M$ maps a point $x \in M$ to its image along the integral curve from time $s$ to time $t$ [41, Th. 9.48], which we denote by $\psi_{t, s}(x)$. The map $\psi$ has similar properties to the map $\phi$ :

- The map $\psi_{t, s}: M \rightarrow M$ is a (local) diffeomorphism for all $s, t \in J$.

- For all $s, t_{1}, t_{2} \in J, x \in M, \psi_{t_{2}, t_{1}}\left(\psi_{t_{1}, s}(x)\right)=\psi_{t_{2}, s}(x), \psi_{s, s}(x)=x$. The inverse of $\psi_{t, s}$ is $\psi_{s, t}$, i.e., $\psi_{t, s}^{-1}\left(\psi_{t, s}(x)\right)=\psi_{s, t}\left(\psi_{t, s}(x)\right)=x$. - The linear map d $\psi_{t, s}: T_{x} M \rightarrow T_{\psi_{t, s}(x)} M$, called the differential (the push-forward) of $\psi_{t, s}$, is an isomorphism between the tangent spaces at each $x \in M$ and $\psi_{t, s}(x) \in M$, for each $s, t \in J$. d $\psi_{t, s}$ maps tangent vectors to all possible curves through a point $x \in M$ to the corresponding tangent vectors of the images of these curves under the diffeomorphism $\psi_{t, s}$, through the point $\psi_{t, s}(x) \in M$.

We note that the notation $\psi_{t, s}(x)$ can of course also be consistently used for the case of time-independent flow. In that case, $\psi_{t, s}(x)=\phi_{t-s}(x)$.

\section{U Pushforwards and Pullbacks}

A smooth map $\phi: M \rightarrow M$ induces for each $x \in M$ a linear map

$$
\begin{aligned}
(\mathrm{d} \phi)_{x}: T_{x} M & \rightarrow T_{\phi(x)} M, \\
\mathbf{x} & \mapsto(\mathrm{d} \phi)_{x}(\mathbf{x}),
\end{aligned}
$$

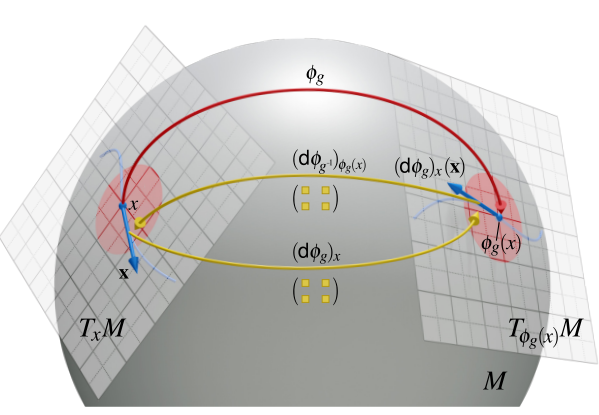

Fig. 13. Pushforward and pullback of a diffeomorphism $\phi_{g}$ are linear maps between the tangent spaces $T_{x} M$ and $T_{\phi_{g}(x)} M$, and cotangent spaces $T_{\phi_{g}(x)}^{*} M$ and $T_{x}^{*} M$, respectively. The pushforward $\left(\mathrm{d} \phi_{g}\right)_{x}$ maps a tangent vector $\mathbf{x} \in T_{x} M$ to the vector $\left(\mathrm{d} \phi_{g}\right)_{x}(\mathbf{x}) \in T_{\phi_{g}(x)} M$. The pullback $\phi_{g}^{*}$ maps a covector (1-form) $\omega \in T_{\phi_{g}(x)}^{*} M$ to the covector (1-form) $\phi_{g}^{*} \omega \in T_{x}^{*} M$. 
called the differential or pushforward, from the tangent space at $x$ to the tangent space at $\phi(x)$.

This is illustrated geometrically in Fig. 13: Choosing a smooth curve through the point $x \in M$ defines a tangent vector $\mathbf{x} \in T_{x} M$. The map $\phi$ maps this smooth curve to another smooth curve through the point $\phi(x) \in M$, defining the tangent vector $(\mathrm{d} \phi)_{x}(\mathbf{x}) \in T_{\phi(x)} M$.

The differential of a smooth map allows to pushforward single tangent vectors to other points on the manifold. Our definition of objectivity requires to pushforward whole vector fields (Sec. 5.2). This is not possible with an arbitrary smooth map $\phi$, as this fails to define tangent vectors at points not hit by $\phi$ ( $\phi$ is not onto), or might define tangent vectors ambiguously at points hit several times ( $\phi$ is not one-to-one).

Diffeomorphisms. However, if a smooth map $\phi$ happens to be both one-to-one and onto, then it uniquely defines another vector field with the tangent vectors being the pointwise pushforwards [41, Proposition 8.10]. Such a smooth map $\phi$ that has an inverse, if this inverse is also smooth, is called a diffeomorphism. That is, we can use a map $\phi$ to pushforward a whole vector field precisely when $\phi$ is a diffeomorphism.

Pullbacks. The corresponding concept to the pushforward of a vector field is the pullback of a covector (1-form) field [41, Ch. 11]. The pullback $\phi^{*}$ of a covector field is defined as

$$
\begin{aligned}
\left(\phi^{*}\right)_{x}: T_{\phi(x)}^{*} M & \rightarrow T_{x}^{*} M, \\
\omega & \mapsto\left(\phi^{*}\right)_{x}(\omega) .
\end{aligned}
$$

Since $\left(\phi^{*}\right)_{x}(\omega)$ is a covector (1-form), in order to define it we have to specify how it acts on a vector $\mathbf{x} \in T_{x} M$. We use the pushforward and the fact that $\omega$ is a covector of the tangent space at $\phi(x)$ :

$$
\left(\phi^{*}\right)_{x}(\omega)(\mathbf{x}):=\omega\left((\mathrm{d} \phi)_{x}(\mathbf{x})\right) .
$$

In contrast to vector fields, covector fields always pull back to covector

\begin{tabular}{|c|c|}
\hline$M, N$ & (smooth) manifolds \\
\hline$(M, \mathbf{g})$ & Riemannian manifold $M$ with metric $\mathbf{g}$ \\
\hline$(U, \varphi)$ & chart on manifold $M ; U \subset M$, chart map $\varphi$ \\
\hline$\left(U_{\alpha}, \varphi_{\alpha}\right)$ & atlas of charts; chart index $\alpha$ from index set \\
\hline$\varphi$ & chart map $\varphi: U \rightarrow \mathbb{R}^{n}$ for $n$-dim. manifold $M$ \\
\hline$x^{i}$ & coordinate functions for chart; $x^{i}: U \rightarrow \mathbb{R}$ \\
\hline$u, v$ & alternative naming for coordinate functs. on 2-manifold \\
\hline$C^{\infty}(M)$ & space of smooth functions on manifold $M$ \\
\hline$T_{x} M$ & tangent space at $x \in M$ \\
\hline$T_{x}^{*} M$ & cotangent space at $x \in M$ \\
\hline$\stackrel{\imath}{T M}$ & tangent bundle of manifold $M$ \\
\hline$T^{*} M$ & cotangent bundle of manifold $M$ \\
\hline $\mathfrak{X}(M)$ & space of vector fields on manifold $M$ \\
\hline$\Omega^{1}(M)$ & space of covector fields (1-forms) on manifold $M$ \\
\hline $\mathbf{e}_{i}$ & basis vector fields/bases in each tangent space $T_{x} M$ \\
\hline$\omega^{i}$ & basis covectors/1-forms (dual to $\left\{\mathbf{e}_{i}\right\}$ ) \\
\hline $\mathbf{e}_{i} \otimes \omega^{j}$ & basis of a $\left(\begin{array}{l}1 \\
1\end{array}\right)$ tensor \\
\hline $\mathbf{e}_{i} \otimes \mathbf{e}_{j}$ & basis of a $\left(\begin{array}{l}2 \\
0\end{array}\right)$ tensor \\
\hline$\omega^{i} \otimes \omega^{j}$ & basis of a $\left(\begin{array}{l}0 \\
2\end{array}\right)$ tensor \\
\hline $\mathbf{g},\langle\cdot, \cdot\rangle$ & metric (on tangent spaces of manifold $M$ ) \\
\hline $\begin{array}{l}g_{i j} \\
\phi\end{array}$ & $\begin{array}{l}\text { compts. of metric in basis }\left\{\omega^{i} \otimes \omega^{j}\right\} ; g_{i j}:=\left\langle\mathbf{e}_{i}, \mathbf{e}_{j}\right\rangle \\
\text { flow of a vector field }\end{array}$ \\
\hline$\phi_{t}$ & one-parameter group generated by the flow $\phi$ \\
\hline$\Phi$ & Lie group action on manifold $M$ \\
\hline$\phi_{g}$ & diffeomorphism generated by Lie group element $g \in G$ \\
\hline $\mathrm{d} \phi_{g}$ & pushforward/differential (lin. map betw. tang. spaces) \\
\hline$\phi_{g}^{*}$ & pullback of $\phi_{g}$ (linear map between cotangent spaces) \\
\hline$\Xi$ & Lie algebra action on manifold $M$ \\
\hline$t \mapsto g(t)$ & path through Lie group $G ; g(t) \in G$ \\
\hline$t \mapsto X(t)$ & path through Lie algebra $\mathfrak{g} ; X(t) \in \mathfrak{g}$ \\
\hline$t \mapsto \phi_{g(t)}$ & diffeomorphisms corresponding to path $t \mapsto g(t) \in G$ \\
\hline$\partial_{i}, \partial_{\mathbf{e}_{i}}$ & partial derivative in direction $\mathbf{e}_{i}$ \\
\hline$\nabla_{i}, \nabla_{\mathbf{e}_{i}}$ & covariant derivative in direction $\mathbf{e}_{i}$ \\
\hline$\Gamma_{j k}^{i}$ & Christoffel symbols; with respect to a basis $\left\{\mathbf{e}_{i}\right\}$ \\
\hline$\partial_{j} v^{i}$ & partial derivatives of vector compts. $v^{i}$ (not a tensor!) \\
\hline$\nabla \mathbf{v}$ & velocity gradient tensor (field) of vector field $\mathbf{v}$ \\
\hline$\nabla_{j} v^{i}$ & components of $\nabla \mathbf{v}$ with respect to a basis $\left\{\mathbf{e}_{i} \otimes \omega^{j}\right\}$ \\
\hline$\nabla_{j} v_{i}$ & comp. of cov. deriv. of cov. field $v_{i} \omega^{i}$; basis $\left\{\omega^{i} \otimes \omega^{j}\right\}$ \\
\hline $\mathbf{u}$ & observer velocity field \\
\hline $\mathscr{L}_{\mathbf{u}}$ & (autonomous) Lie derivative with resp. to the field $\mathbf{u}$ \\
\hline$L_{\mathbf{u}}$ & time-dependent Lie derivative with resp. to the field $\mathbf{u}$ \\
\hline$G$ & Lie group \\
\hline $\mathfrak{g}$ & Lie algebra of Lie group $G$ \\
\hline$g$ & Lie group element $g \in G$ \\
\hline$X$ & Lie algebra Element $X \in \mathfrak{g}$ \\
\hline $\operatorname{Isom}(M)$ & isometry group of manifold $M$; a Lie group \\
\hline $\mathfrak{i s o m}(M)$ & Lie algebra of the isometry group of manifold $M$ \\
\hline $\mathrm{O}(n)$ & orthogonal group \\
\hline $\mathrm{SO}(n)$ & special (det $g=1)$ orthogonal group (rotations) \\
\hline $\mathfrak{s o}(n)$ & Lie algebra of $\mathrm{SO}(n)$; all $X \in \mathfrak{s o}(n)$ are anti-symmetric \\
\hline $\mathrm{T}(n)$ & translation group of $\mathbb{R}^{n}$ \\
\hline $\mathrm{E}(n)$ & Euclidean group of $\mathbb{R}^{n} ; \mathrm{E}(n)=\mathrm{O}(n) \ltimes \mathrm{T}(n)$ \\
\hline $\mathrm{SE}(n)$ & special Euclidean group; $\mathrm{SE}(n)=\mathrm{SO}(n) \ltimes \mathrm{T}(n)$ \\
\hline$K \mathbf{u}$ & Killing operator applied to $\mathbf{u}$ \\
\hline & Killing energy (density) of $\mathbf{u}$ at point $x \in M$ \\
\hline $\int_{M} E \mathbf{u} \mathrm{d} A$ & Killing energy of $\mathbf{u}$ on $M$; area element $\mathrm{d} A$ \\
\hline$\langle\mathbf{T}, \mathbf{S}\rangle_{\mathbf{g}}$ & tensor inner product of $\mathbf{T}$ and $\mathbf{S}$ with resp. to metric $\mathbf{g}$ \\
\hline$\|\mathbf{T}\|_{\mathbf{g}}$ & tensor norm of $\mathbf{T}$ with respect to metric $\mathbf{g}$ \\
\hline$(D / D t)$ & material time derivative \\
\hline$(\mathscr{D} / \mathscr{D} t)$ & observed time derivative (wrt. an observer field $\mathbf{u}$ ) \\
\hline
\end{tabular}
fields, even when the map $\phi$ is not a diffeomorphism.

\section{Notation TABLe}

